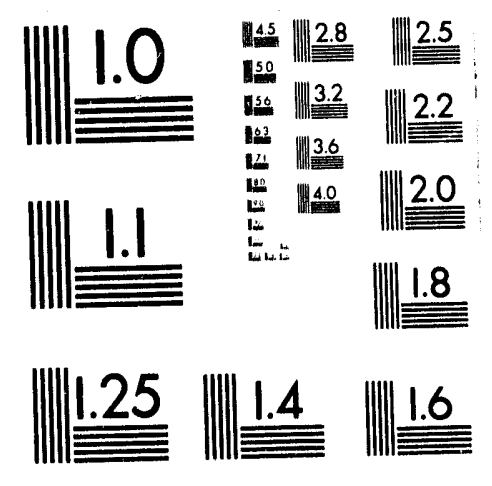



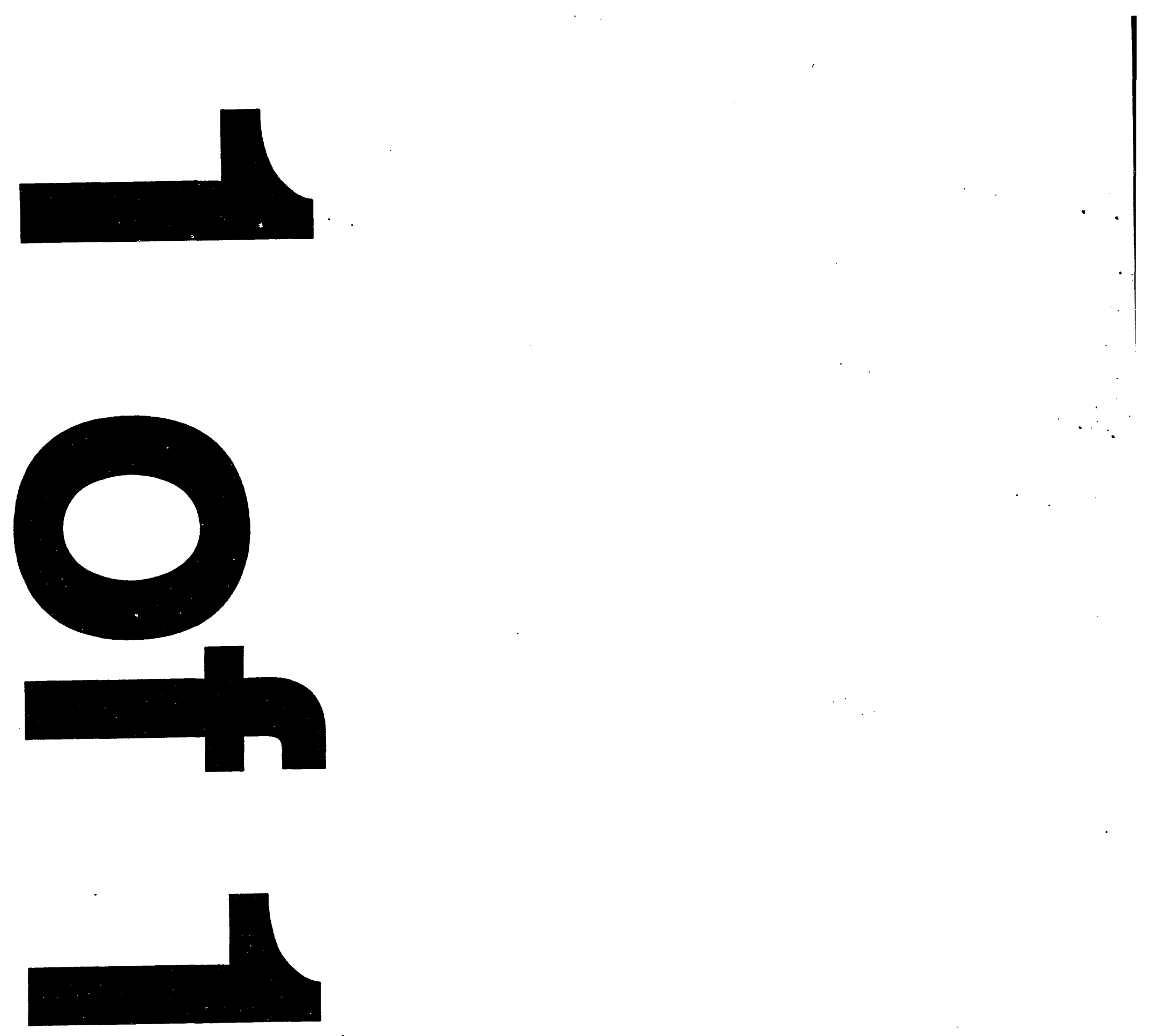
SAND92-1911

Distribution

Unlimited Release

Printed June 1993

Category UC-721

\title{
Permeability and Hydraulic Diffusivity of Waste Isolation Pilot Plant Repository Salt Inferred from Small-Scale Brine Inflow Experiments
}

\author{
D. F. McTigue \\ Thermal and Fluid Engineering Department \\ Sandia National Laboratories \\ Albuquerque, New Mexico 87185
}

\begin{abstract}
Brine seepage to 17 boreholes in salt at the Waste Isolation Pilot Plant (WIPP) facility horizon has been monitored for several years. A simple model for one-dimensional, radial, darcy flow due to relaxation of ambient pore-water pressure is applied to analyze the field data. Fits of the model response to the data yield estimates of two parameters that characterize the magnitude of the flow and the time scale over which it evolves. With further assumptions, these parameters are related to the permeability and the hydraulic diffusivity of the salt. For those data that are consistent with the model prediction, estimated permeabilities are typically $10^{-22}$ to $10^{-21} \mathrm{~m}^{2}$. The relatively small range of inferred permeabilities reflects the observation that the measured seepage fluxes are fairly consistent from hole to hole, of the order of $10^{-10} \mathrm{~m} / \mathrm{s}$. Estimated diffusivities are typically $10^{-10}$ to $10^{-8} \mathrm{~m}^{2} / \mathrm{s}$. The greater scatter in inferred hydraulic diffusivities is due to the difficulty of matching the idealized model history to the observed evolution of the flows. The data obtained from several of the monitored holes are not consistent with the simple model adopted here; material properties could not be inferred in these cases.
\end{abstract}

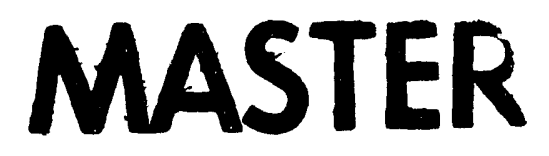




\section{CONTENTS}

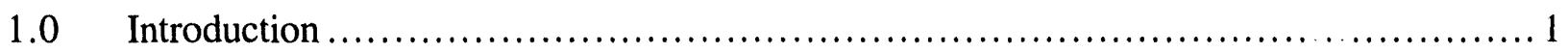

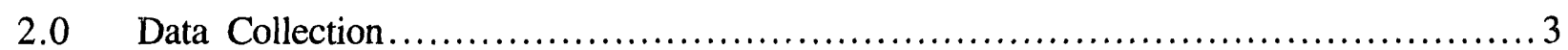

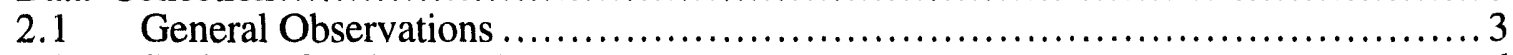

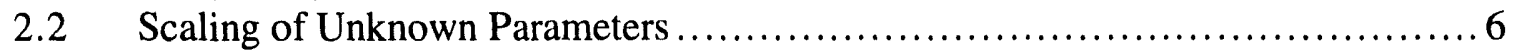

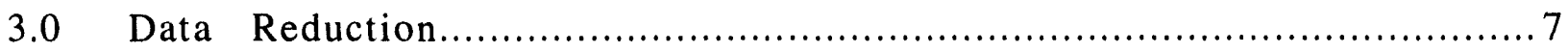

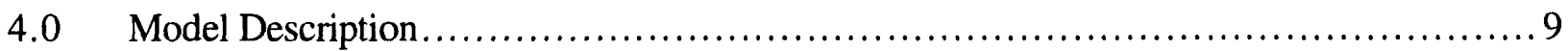

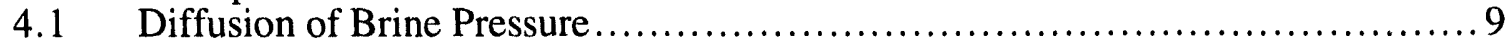

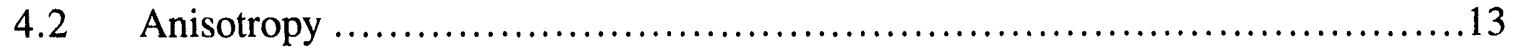

5.0 Radial Flow to an Open Borehole .................................................... 15

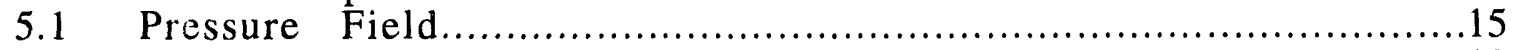

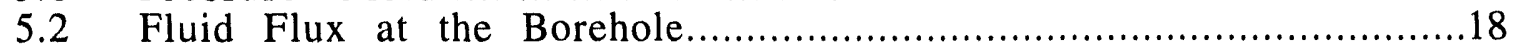

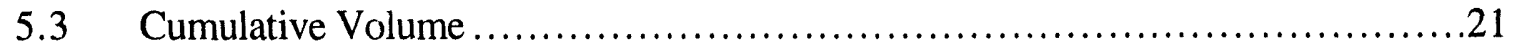

$5.4 \quad$ Late-Time, Asymptotic Flux ................................................23

5.5 Flow Limited to Discrete Horizons.................................................

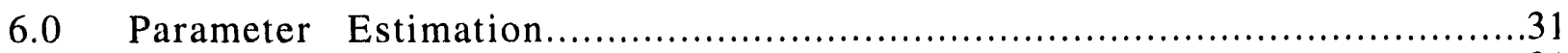

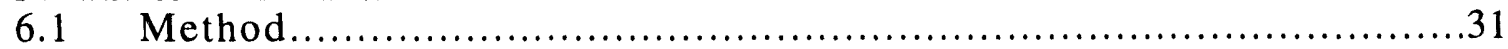

6.2 Fits Based on the Full Flux Histories............................................

6.2.1 Deleted Data and Other Data Reduction.........................................33

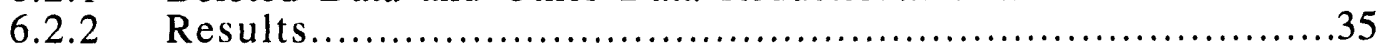

6.3 Fits Based on the Full Cumulative Volume Histories ..........................45

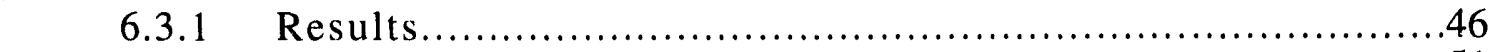

6.4 Fits Based on the Late-Time Flux Histories..........................................51

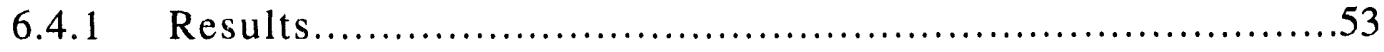

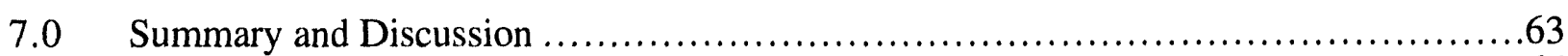

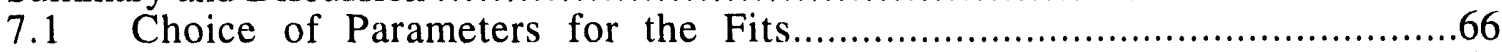

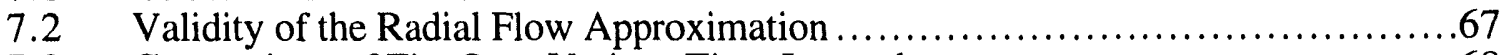

7.3 Comparison of Fits Over Various Time Intervals ............................68

7.4 Comparison of Fits by Various Methods .....................................70

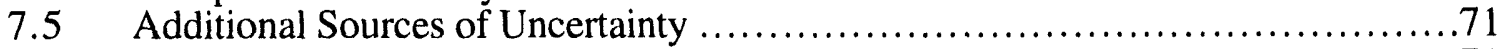

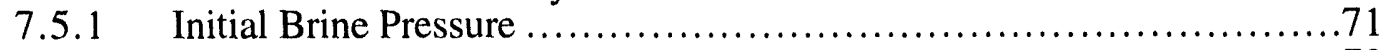

7.5.2 Brine Density .................................................... 72

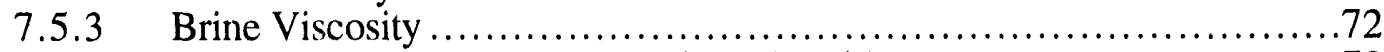

7.6 Expected Capacitance for an Elastic Matrix and Fluid .........................73

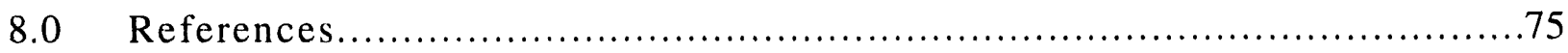




\section{FIGURES}

2-1 Locations of the boreholes considered in this study .......................................... 4

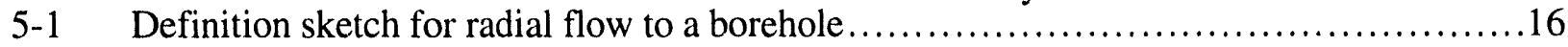

5-2 Pore pressure profiles at various times based on Equation (31); radial coordinate, $r$, is normalized by borehole radius, $a$; pressure, $p$, is normalized by initial value $p_{\infty} \ldots \ldots .19$

5-3 Fluid flux at a borehole, evaluated from "exact" solution, Equation (35) $\ldots \ldots \ldots \ldots \ldots \ldots \ldots .25$

5-4 Comparison of late-time approximations (Equation 41) and "exact" solution

(Equation 35) for fluid flux at a borehole; time, $t$, is normalized by characteristic

diffusion time, $a^{2} / c$; flux, $\left|q_{r}\right|(a, t)$, is normalized by $q_{0}=k p_{\infty} / \mu a . \ldots \ldots \ldots \ldots \ldots \ldots . .26$

5-5 Comparison of "exact" and late-time, asymptotic solutions for inverse of borehole flux; time, $t$, is normalized by characteristic diffusion time, $a^{2} / c$; flux, $\left|q_{r}\right|(a, t)$, is

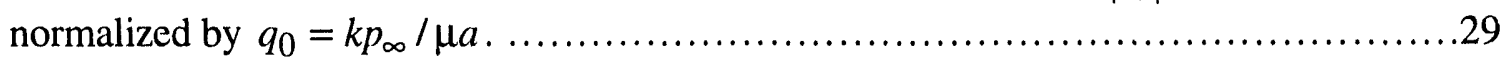

6-1 Comparison of model fit to data for full flux history, hole DBT10 ......................37

6-2 Comparison of model fit to data for full flux history, hole DBT11 $\ldots \ldots \ldots \ldots \ldots \ldots \ldots \ldots . \ldots 37$

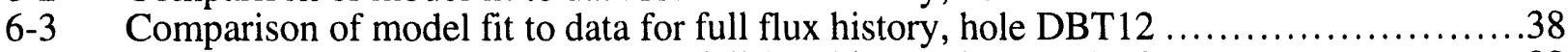

6-4 Comparison of model fit to data for full flux history, hole DBT13 $\ldots \ldots \ldots \ldots \ldots \ldots \ldots \ldots . \ldots 38$

6-5 Comparison of model fit to data for full flux history, hole DBT14A ....................39

6-6 Comparison of model fit to data for full flux history, hole DBT14B ....................39

6-7 Comparison of model fit to data for full flux history, hole DBT15A ......................40

6-8 Comparison of model fit to data for full flux history, hole DBT 15B $\ldots \ldots \ldots \ldots \ldots \ldots \ldots \ldots . \ldots 40$

6-9 Comparison of model fit to data for full flux history, hole DBT31 A $\ldots \ldots \ldots \ldots \ldots \ldots \ldots \ldots 41$

6-10 Comparison of model fit to data for full flux history, hole DBT31C $\ldots \ldots \ldots \ldots \ldots \ldots \ldots . \ldots 4$

6-11 Comparison of model fit to data for full flux history, hole DBT32C $\ldots \ldots \ldots \ldots \ldots \ldots \ldots \ldots .42$

6-12 Comparison of model fit to data for full flux history, hole L4B01 .....................4.

6-13 Comparison of model fit to data for full flux history, hole QPB01 $\ldots \ldots \ldots \ldots \ldots \ldots \ldots \ldots . \ldots 4$

6-14 Comparison of model fit to data for full flux history, hole QPB02 $\ldots \ldots \ldots \ldots \ldots \ldots \ldots \ldots .43$

6-15 Comparison of model fit to data for full flux history, hole QPB03 ....................44

6-16 Comparison of model fit to data for full flux history, Room D holes grouped .............44

6-17 Comparison of model fit to data for full flux history, Room Q Access Drift holes grouped...................................................................... 45

6-18 Comparison of model fit to data for cumulative volume history, hole DBT10...............47

6-19 Comparison of model fit to data for cumulative volume history, hole DBT11 1..............47

6-20 Comparison of model fit to data for cumulative volume history, hole DBT12...............48

6-21 Comparison of model fit to data for cumulative volume history, hole DBT13..............48

6-22 Comparison of model fit to data for cumulative volume history, hole DBT14A.............49

6-23 Comparison of model fit to data for cumulative volume history, hole DBT15...............49

6-24 Comparison of model fit to data for cumulative volume history, hole L4B01 ............50

6-25 Comparison of model fit to data for cumulative volume history, hole QPB01................50

6-26 Comparison of model fit to data for cumulative volume history, hole QPB02...............51

6-27 Comparison of model fit to data for late-time flux, hole DBT10. Points included in linear regression are shaded ...................................................... 54

6-28 Comparison of model fit to data for late-time flux, hole DBT11. Points included in linear regression are shaded .................................................... 54

6-29 Comparison of model fit to data for late-time flux, hole DBT 12. Points included in

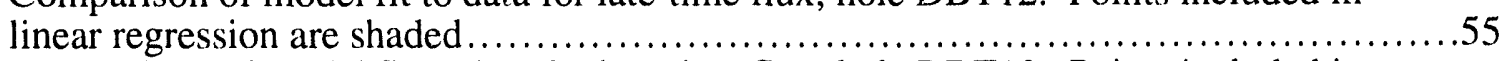

6-30 Comparison of model fit to data for late-time flux, hole DBT 13. Points included in

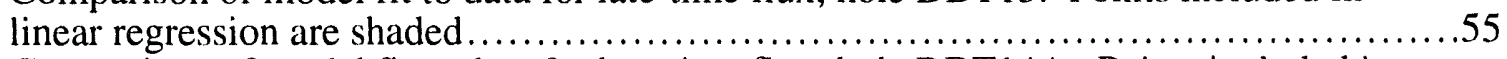

6-31 Comparison of model fit to data for late-time flux, hole DBT14A. Points included in

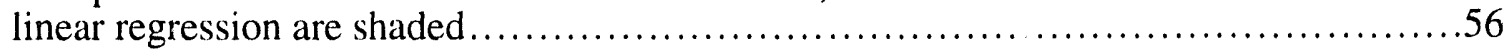




\section{FIGURES (CONTINUED)}

6-32 Comparison of model fit to data for late-time flux, hole DBT15A. Points included in

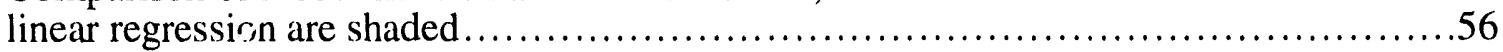

6-33 Comparison of model fit to data for late-time flux, hole DBT15B. All points shown

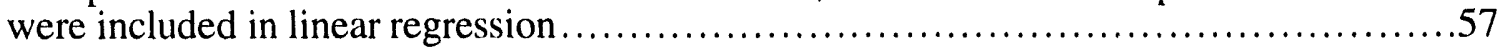

6-34 Comparison of model fit to data for late-time flux, hole DBT31A. All points shown were included in linear regression ...............................................57

6-35 Comparison of model fit to data for late-time flux, hole DBT32C. Points included in linear regression are shaded ...................................................5

6-36 Comparison of model fit to data for late-time flux, hole LAB01. Points included in

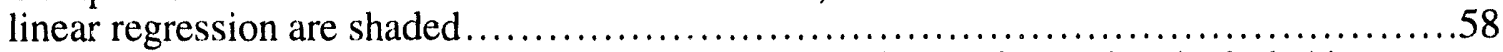

6-37 Comparison of model fit to data for late-time flux, hole L4X01. Points included in

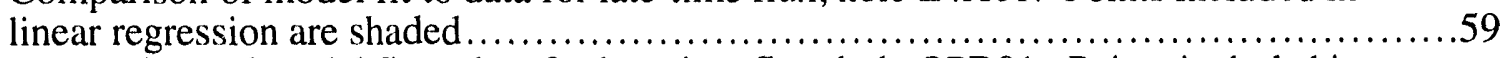

6-38 Comparison of model fit to data for late-time flux, hole QPB01. Points included in linear regression are shaded ..................................................59

6-39 Comparison of model fit to data for late-time flux, hole QPB02. Points included in linear regression are shaded ...................................................60

6-40 Comparison of model fit to data for late-time flux, hole QPB03. Points included in linear regression are shaded .....................................................6

7-1 Histogram of permeabilities; fits to full flux histories (Table 6-1). Bins centered on $10^{\mathrm{n}}$ include values from 0.5 to $5.0 \times 10^{\mathrm{n}}$. Mean value of $k$ is -21.3 ; standard deviation of $k$ is 0.9

7-2 Histogram of hydraulic diffusivities; fits to full flux histories (Table 6-1). Bins centered on $10^{\mathrm{n}}$ include values from 0.5 to $5.0 \times 10^{\mathrm{n}}$. Mean value of $c$ is -9.3 ; standard deviation of $c$ is 1.8

7-3 Histogram of capacitances; fits to full flux histories (Table 6-1). Bins centered on $10^{\text {n }}$ include values from 0.5 to $5.0 \times 10^{\mathrm{n}}$. Mean value of $C$ is -9.4 ; standard deviation of $C$ is 1.2 . . .

7-4 Comparison of permeability values $\left(\mathrm{m}^{2}, \times 10^{-21}\right)$ determined by various methods Perfect correlations lie on the solid line ...........................................70

7-5 Comparison of capacitance values $\left(\mathrm{Pa}^{-1}, \times 10^{-9}\right)$ determined by various methods Perfect correlations lie on the solid line......

\section{TABLES}

2-1 Borehole Characteristics.......................................................... 5

5-1 Pressure Profiles at Various Times Based on Equation (31) ..............................20

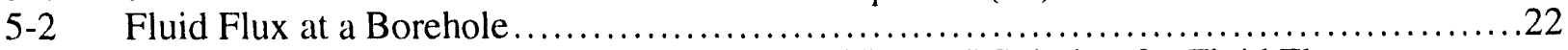

5-3 Comparison of Late-time Approximations and "Exact" Solution for Fluid Flux at a

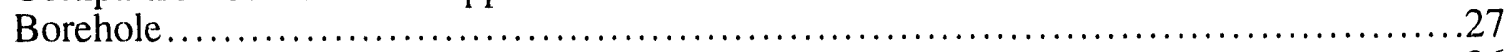

6-1 Parameters Derived from Fits to Full Flux .......................................... 36

6-2 Parameters Derived from Fits to Cumulative Volume...........................................52

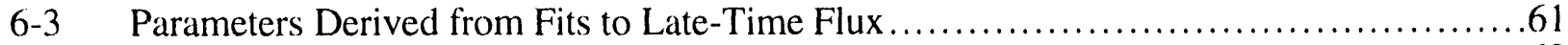

7-1 Diffusion Length Scales for Various Diffusivities..........................................68

7-2 Parameters for DBT10 by Various Fitting Schemes .................................6 69

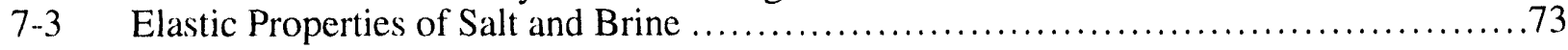




\subsection{INTRODUCTION}

Measurable accumulations of brine have been observed in some drillholes and excavations at the Waste Isolation Pilot Plant (WIPP) facility horizon for a number of years. Because the presence of brine may affect backfill consolidation, gas generation, room closure, and the performance of the seals and waste package, a predictive model is desired. A successful model would allow calculations of the brine inflow rate, or, equivalently, the cumulative volume of brine as it evolves through time for a given room geometry. Any model that purports to represent the essential brine transport process(es) will require as input numerical values of various material properties. The purpose of this report is to document a first attempt to infer such parameters from field data collected over a period of several years.

The study invokes one of the simplest models that might be assumed to represent the flow of brine through salt. The salt is represented as a fluid-saturated, porous medium in which the flux of brine is assumed to be governed by Darcy's law. Classical arguments lead to a description of brine transport in the form of a linear diffusion problem. This parallels conventional problems of hydrological flows in confined aquifers. An exact solution is known for the transient, onedimensional (radial) flow to a long borehole in an unbounded domain initially at uniform pressure. The behavior of the solution depends on two groups of parameters. Fits of the analytical function to the field data thus yield estimates of two unknown parameters that characterize the system. In the present case, three unknown parameters arise in the analysis: the initial pressure of the brine, the permeability of the salt, and the hydraulic diffusivity of the brine in the salt. (Various combinations of these parameters are equally valid choices of constants to be determined by fitting the data.) Independent measurements have indicated typical values for the initial brine pressure in the salt, which can be used to isolate values for permeability and diffusivity.

The field data used to infer the hydraulic properties of the salt are from the small-scale brineinflow experiments conducted in the WIPP facility beginning in September 1987. The datacollection program is detailed in a report by Finley et al. (1992), which presents and discusses data collected through early June 1991. The analysis reported here considers only the data obtained through mid-January 1990; later data were not available when this analysis was performed. The experiments consist of regular collection and weighing of the brine (if any) accumulated in each of 17 boreholes. This provides a measure of the integrated brine flux over the collection interval. A measure of the average flow rate can also be obtained simply by dividing the volume collected by the sampling interval. The observed cumulative volume and flux histories provide the data to 
which the model is fit. The fits seek the set of unknown parameters that minimizes the difference between the observations and the model calculations.

The model on which the interpretation of these data is based is highly idealized: onedimensional, radial, darcy flow, assuming a homogeneous, isotropic medium and a uniform initial brine pressure. In this context, the model can be fit meaningfully only to "well-behaved" data (i.e., holes that exhibit a relatively high initial flux and a smooth, monotonic decay). Nonetheless, it is worthwhile to see the extent to which the salt appears to respond as a classical hydrological system. Furthermore, in those cases where the model appears to yield a reasonable approximation to the observed behavior, the fitting exercise yields estimates of critical material properties.

The exercise also reveals some limitations of the model. While many of the monitored holes show behavior consistent with the model, some do not. Evidently, more complex processes not considered here influence the yield of brine in some holes. Even in the context of the classical model, such phenomena as heterogeneity and anisotropy in the material properties, nonuniform initial pressure, and the multi-dimensionality of the true configuration of the monitored holes may exert significant influence. In addition, intersection of the holes with discrete fractures would introduce different boundary conditions for which the present analysis does not apply. Beyond these complications are the possibilities of more complex interactions between the deformation of the salt and the brine flow, as well as multiphase flow (e.g., exsolution of dissolved gases, imbibition of room air, seepage of brine through unsaturated salt, etc.).

Section 2 of this report summarizes the data collection methods and general observations about the brine inflow. The data reduction scheme is described in Section 3. Section 4 provides the background of the model that is assumed to represent the transport of brine in salt. The solution for flow to a borehole and the numerical evaluation method are described in Section 5. The parameter-estimation scheme and the results for various model approaches are described in Section 6. Finally, the results are summarized and discussed in Section 7. 


\subsection{DATA COLLECTION}

\subsection{General Observations}

This report records preliminary reduction of data from boreholes drilled in salt in the WIPP facility horizon and provides a brief discussion of their interpretation in view of a classical darcyflow model. Brine flow to 17 boreholes in Room D, Room L4, and the Room Q Access Drift has been monitored since January 1987, May 1989, and April 1989, respectively. This report treats only data collected until January 1990, covering a period of up to 850 days. A detailed description of the data collection process and the results through June 1991 is given by Finley, et al. (1992). The stratigraphy penetrated by the 17 holes is shown in Figure 1-1. Their dimensions and histories are summarized in Table 1-1. Holes that were extended in length or enlarged in radius are designated here by the letters A, B, and C for each successive stage. The hole locations in each room are shown in Finley et al. (1992).

Although there is considerable variability in the observed response of the holes, there are significant similarities. Except for the two inclined holes DBT16 and DBT17, all monitored holes yielded some brine, with maximum total mass flow rates of 2 to $25 \mathrm{~g} / \mathrm{d}$ during the period considered in this report. The single exception was hole QPB02, which yielded over $100 \mathrm{~g} / \mathrm{d}$ for the first 100 days.

In many cases, the flow rate declined in a fairly smooth fashion over time, which is the expected behavior of an open borehole in a classical hydrological system (see Section 5.2). Holes DBT10 to DBT15 showed this response at early time, although the flow to DBT14 actually increased with time after about 100 days. All these holes showed somewhat more erratic behavior after several hundred days, with occasional increases in flow rate (DBT12) or an apparent levelingoff of the flow (DBT13). Holes DBT31 and DBT32 were initially 4-inch-diameter (radius $a=$ $0.051 \mathrm{~m}$ ) holes; DBT31 showed a decline in flow at early time, while DBT32 was erratic. After extension and then enlargement, these holes exhibited erratic responses again, although tentative information was extracted from the period of declining flow in DBT32 at about 450 days. 




\section{Legend}

\# Halite

NInhydrite

$\left[\begin{array}{l}x \\ x \\ x\end{array}\right]$ Polyhalitic Halite

E: Argillaceous Halite

Clay Seam

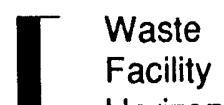

Vertical Scale

(No Horizontal Scale)

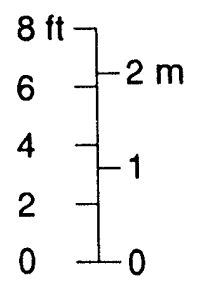

Figure 2-1. Locations of the boreholes considered in this study (from Finley, et al., 1992). 
Table 2-1. Borehole Characteristics

\begin{tabular}{|c|c|c|c|c|}
\hline \hline Hole & Operation & Date & $\begin{array}{c}\text { Length } \\
(\mathrm{m})\end{array}$ & $\begin{array}{c}\text { Radius } \\
(\mathrm{m})\end{array}$ \\
\hline DBT10 & drilled & $9 / 18 / 87$ & 5.334 & 0.051 \\
DBT11 & drilled & $9 / 23 / 87$ & 4.633 & 0.051 \\
DBT12 & drilled & $9 / 22 / 87$ & 3.688 & 0.051 \\
DBT13 & drilled & $9 / 17 / 87$ & 2.804 & 0.051 \\
DBT14A & drilled & $9 / 14 / 87$ & 2.591 & 0.051 \\
DBT14B & extended & $6 / 30 / 88$ & 5.608 & 0.051 \\
DBT15A & drilled & $9 / 15 / 87$ & 2.743 & 0.051 \\
DBT15B & extended & $7 / 5 / 88$ & 5.791 & 0.051 \\
DBT16A & drilled & $9 / 25 / 87$ & 2.540 & 0.051 \\
DBT16B & extended & $7 / 27 / 88$ & 5.169 & 0.051 \\
DBT17A & drilled & $9 / 29 / 87$ & 2.540 & 0.051 \\
DBT17B & extended & $7 / 28 / 88$ & 5.436 & 0.051 \\
DBT31A & drilled & $9 / 8 / 87$ & 2.134 & 0.051 \\
DBT31B & extended & $2 / 5 / 88$ & 4.877 & 0.051 \\
DBT31C & enlarged & $5 / 18 / 88$ & 5.639 & 0.457 \\
DBT32A & drilled & $9 / 10 / 87$ & 2.896 & 0.051 \\
DBT32B & extended & $2 / 9 / 88$ & 5.664 & 0.051 \\
DBT32C & enlarged & $5 / 24 / 88$ & 5.664 & 0.457 \\
L4B01 & drilled & $5 / 16 / 89$ & 5.791 & 0.051 \\
L4X01 & drilled & $5 / 4 / 89$ & 5.715 & 0.457 \\
QPB01 & drilled & $4 / 18 / 89$ & 3.048 & 0.025 \\
QPB02 & drilled & $4 / 18 / 89$ & 3.099 & 0.025 \\
QPB03 & drilled & $4 / 18 / 89$ & 3.099 & 0.025 \\
QPB04 & drilled & $5 / 1 / 89$ & 3.073 & 0.025 \\
QPB05 & drilled & $5 / 1 / 89$ & 3.099 & 0.025 \\
\hline
\end{tabular}

Hole L4B01 (radius $0.051 \mathrm{~m}$ ) exhibited a smooth decay in flux that allows a fit of the simple model. The nearby hole L4X01, situated in the same stratigraphic horizon and of the same length and angle, is of much greater radius, $0.46 \mathrm{~m}$. Hole L4X01 showed a high initial flux and subsequent decay, but the decay was quite rapid, with the flux reaching zero at about 170 days. 
Because the observed flux-decay rate to L4X01 does not decrease continuously, a fit of the model could not be obtained.

Among the boreholes in the Room Q access drift, hole QPB01 shows a response over the considered time interval that is most consistent with the idealized model and allows a fit to determine material parameters. As noted above, hole QPB02 yielded a much greater quantity of brine than any other hole monitored in this study. It showed a relatively smooth decay in flux over the first 46 days, followed by a steeper decline until about 200 days, when the flux was at least two orders of magnitude smaller than that calculated from the earliest observation. A discrete fracture with a detectable offset was identified by a borehole camera in QPB02 in March 1991 (Finley et al., 1992); this provides one possible explanation for the anomalously large flux. Hole QPB03 showed relatively small changes in flux over time, but a fit of the model was obtained. Holes QPB04 and QPB05 yielded no brine for the first several samplings, and they exhibited rather erratic accumulations. No fits could be obtained for these data.

\subsection{Scaling of Unknown Parameters}

Simple scaling arguments based on the darcy flow model, which are developed in more detail in Sections 4 and 5, indicate the orders of magnitude of the parameters of interest. The permeability is shown to scale like $q_{0} \mu a / p_{\infty}$, where $q_{0}$ is the fluid flux, $\mu$ is the fluid viscosity, $a$ is the borehole radius, and $p_{\infty}$ is the initial pressure. Typical fluxes, based on the total volume flow rates divided by borehole wall areas, are of the order of $q_{0} \sim 10^{-10} \mathrm{~m} / \mathrm{s}$; the brine viscosity is of the order of $\mu \sim 10^{-3} \mathrm{~Pa} \cdot \mathrm{s}$; initial pressures are believed to be of the order of $p_{\infty} \sim 10^{7} \mathrm{~Pa}$. The scaling argument thus indicates permeabilities of the order of $10^{-21} \mathrm{~m}^{2}$, and this is borne out by the more elaborate fitting exercise.

The model also shows that the fluid diffusivity, $c$, scales like $c \sim a^{2} / t_{0}$, where $t_{0}$ is a characteristic time, defined as the time at which the flow has fallen off to $e^{-1}$ times the observed maximum. For those holes that exhibited a smooth decline in flow rate, $t_{0}$ typically ranges from several tens to several hundreds of days, which implies a hydraulic diffusivity of the order of $10^{-10}$ to $10^{-9} \mathrm{~m}^{2} / \mathrm{s}$. This is again borne out by many of the detailed fits, but the diffusivity is rather difficult to determine with confidence from these data. 


\subsection{DATA REDUCTION}

Data were collected for the mass of brine accumulated over a given period between samples. Volumes were computed by dividing the mass by the brine density, taken here to be $1200 \mathrm{~kg} / \mathrm{m}^{3}$. Volumes per unit area of borehole wall were calculated by dividing the volume by the cylindrical surface area of each hole (the end area is neglected), $2 \pi a L$, where $L$ is the length of the hole. Fluxes were calculated by a "centered difference" approximation (i.e., volume per unit area divided by time between samplings with the resulting flux assigned to the midpoint time of the interval). Note that Finley et al. (1992) report mass-flow rates based on a "backward difference" approximation (i.e., mass divided by time between sampling with the resulting mass-flow rate assigned to the time of the measurement).

The foregoing procedure averages the total brine seepage over the entire borehole wall area. The salt intersected by the hole is assumed to be homogeneous, and the seepage is assumed to be uniform along the length of the hole. Thus, parameter estimation based on data treated in this manner yields effective values of the parameters over a sampling scale of the order of the borehole length. In general, the field sampling method cannot discriminate between uniformly distributed flow and localized seepage in discrete horizons intersected by a particular hole. However, in cases where there is reason to believe that the flow is derived principally from some fraction of the borehole length, the appropriate corrections to the parameter estimates are straightforward. This procedure is discussed further in Section 5.5. 


\subsection{MODEL DESCRIPTION}

\subsection{Diffusion of Brine Pressure}

The model that underlies much of classical hydrology results in a linear diffusion equation for the excess pore pressure or, equivalently, hydraulic head in the fluid. Both standard texts and the research literature discuss many different ways of deriving this governing equation (e.g., Bear, 1972). One such derivation is summarized briefly here for completeness, and for reference in the subsequent discussion of results. This particular development is equivalent to, or contains as special cases, most of the classical models.

Conservation of mass for the fluid component of a saturated, porous medium is given by

$$
\frac{\partial \rho}{\partial t}+\nabla \cdot\left(\rho \mathbf{v}_{f}\right)=0
$$

where $\rho$ is the partial density of the fluid, defined as mass of fluid per unit volume of the fluid/solid system, and $\mathbf{v}_{f}$ is the fluid velocity, averaged on a suitable scale to represent the volume flux of fluid per unit area of fluid. It is convenient to decompose the partial density into the product of the porosity, $\phi$, and the material density, $\gamma_{f}$, defined as mass of fluid per unit volume of fluid:

$$
\rho_{f}=\phi \gamma_{f}
$$

Substitution of (2) into (1), rearrangement of terms, and linearization lead to the following statement of the fluid mass balance:

$$
\frac{\partial \phi}{\partial t}+\frac{\phi_{0}}{\gamma_{f_{0}}} \frac{\partial \gamma_{f}}{\partial t}+\nabla \cdot \mathbf{q}+\phi_{0} \nabla \cdot \mathbf{v}_{s}=0
$$

where subscript zeroes indicate constant reference values of the parameters, $\mathbf{v}_{s}$ is the solid skeleton velocity (again averaged on an appropriate scale) and $\mathbf{q}$ is the darcy flux, or "seepage velocity," defined in terms of the relative velocity of the fluid and solid, $\mathbf{q} \equiv \phi_{0}\left(\mathbf{v}_{f}-\mathbf{v}_{s}\right)$. A similar statement of mass conservation for the solid skeleton takes the form: 


$$
-\frac{\partial \phi}{\partial t}+\frac{\left(1-\phi_{0}\right)}{\gamma_{s 0}} \frac{\partial \gamma_{s}}{\partial t}+\left(1-\phi_{0}\right) \nabla \cdot \mathbf{v}_{s}=0,
$$

where $\gamma_{s}$ is the material density of the solid. Equations (3) and (4) sum to give:

$$
\frac{\phi_{0}}{\gamma_{f 0}} \frac{\partial \gamma_{f}}{\partial t}+\frac{\left(1-\phi_{0}\right)}{\gamma_{s 0}} \frac{\partial \gamma_{s}}{\partial t}+\nabla \cdot \mathbf{q}+\nabla \cdot \mathbf{v}_{s}=0
$$

For incompressible constituents, $\gamma_{f}=\gamma_{f 0}$ and $\gamma_{s}=\gamma_{s 0}$ are constants, the first two terms vanish, and Equation (5) simply states that an influx of fluid into a volume element of the porous medium is balanced by dilatation of the solid skeleton, or conversely: an efflux of fluid is balanced by compaction. The more general case given by Equation (5), allowing for compressible constituents, is treated in the remainder of this section.

To complete the model, it is necessary to stipulate constitutive equations that represent the behavior of the materials of interest. In particular, the dependence of $\gamma_{f}, \gamma_{s}, \mathbf{q}$, and $\nabla \cdot \mathbf{v}_{s}$ on the fluid pore pressure $p$ must be given. First, the fluid density is assumed to depend linearly on the pore pressure:

$$
\gamma_{f}=\gamma_{f 0}\left[1+\frac{1}{K_{f}}\left(p-p_{0}\right)\right] \text {, }
$$

where $K_{f}$ is the fluid bulk modulus (the inverse of the fluid compressibility), and $p_{0}$ is the fluid pressure in the reference state. Second, the solid density is assumed to depend on the mean total normal stress, $\sigma$, and the fluid pressure:

$$
\gamma_{s}=\gamma_{s 0}\left\{1-\frac{1}{\left(1-\phi_{0}\right) K_{s}}\left[\left(\sigma-\sigma_{0}\right)+\phi_{0}\left(p-p_{0}\right)\right]\right\},
$$

where $K_{S}$ is the bulk modulus of the solid, and $\sigma_{0}$ is the mean total stress in the reference state. Compression is negative in the sign convention adopted here. The mean stress, $\sigma$, is defined as one-third the trace of the total stress. The mean stress is thus minus the "confining pressure" of traditional usage in soil and rock mechanics. The fluid flux is assumed to follow Darcy's law, which is a statement of the quasi-static balance of momentum for the fluid: 


$$
\mathrm{q}=-\frac{k}{\mu} \nabla\left(p-\gamma_{f 0} g z\right)
$$

where $k$ is the permeability (here assumed to be isotropic), $\mu$ is the fluid viscosity, $g$ is the magnitude of the gravitational acceleration, and the $z$ coordinate is vertical and positive upward. Finally, the dilatation rate, $\nabla . \mathbf{v}_{s}$, is identified (for small deformation) with the rate of change of the volumetric strain, $\varepsilon$, which, in turn, is related to the mean stress and fluid pressure via Hooke's Law for a linearly elastic material:

$$
\nabla \cdot \mathbf{v}_{s} \simeq \frac{\partial \varepsilon}{\partial t}=\frac{1}{K} \frac{\partial}{\partial t}\left[\left(\sigma-\sigma_{0}\right)+\left(1-\frac{K}{K_{s}}\right)\left(p-p_{0}\right)\right]
$$

where $K$ is the bulk modulus of the "drained" $\left(p-p_{0}=0\right)$ porous skeleton.

Substitution of Equations (6) through (9) into Equation (5), along with the assumption of uniform permeability, $k$, and viscosity, $\mu$, gives

$$
C^{\prime} \frac{\partial p}{\partial t}-\frac{k}{\mu} \nabla^{2} p=-B^{\prime} \frac{\partial \sigma}{\partial t}
$$

where

$$
C^{\prime}=\frac{1}{K}\left(1-\frac{K}{K_{s}}\right)+\frac{\phi_{0}}{K_{f}}\left(1-\frac{K_{f}}{K_{s}}\right),
$$

and

$$
B^{\prime}=\frac{1}{K}\left(1-\frac{K}{K_{s}}\right)
$$

The compatibility constraint for a porous, elastic material can be written in the form

$$
\nabla^{2} \sigma=-\frac{2(1-2 v)}{3(1-v)} K B^{\prime} \nabla^{2} p
$$

Equations (10) and (13) can be combined in the form

$$
C \frac{\partial p}{\partial t}-\frac{k}{\mu} \nabla^{2} p=-B\left(\frac{\partial \sigma}{\partial t}-c \nabla^{2} \sigma\right)
$$


where $C$ is the capacitance,

$$
C=C^{\prime}\left[1-\frac{2(1-2 v)}{3(1-v)} \frac{K B^{\prime 2}}{C^{\prime}}\right]
$$

and $B$ is a source coefficient:

$$
B=B^{\prime}\left[1-\frac{2(1-2 v)}{3(1-v)} \frac{K B^{\prime 2}}{C^{\prime}}\right]
$$

The parameter $c$ on the right side of Equation (14) is the hydraulic diffusivity, defined by

$$
c=\frac{k}{\mu C} \text {. }
$$

The quantity $B / C$ is known as Skempton's coefficient and represents the ratio of the pore-pressure increment to the magnitude of the mean stress change under undrained conditions (Rice and Cleary, 1976).

Equations (7), (9), and (13) introduce the mean total stress, $\sigma=\operatorname{tr} \sigma / 3$, as an additional field variable that must be determined as part of any application of the model. In general, this requires solution of the balance of linear momentum, or the "equilibrium" equation of classical elastostatics, $\nabla . \sigma+\rho_{0} g=0$, where $\rho_{0}=\gamma_{f 0}\left(1-\phi_{0}\right)+\gamma_{s 0} \phi_{0}$ is the total density, and $\mathbf{g}$ is the gravitational acceleration vector. In some special cases, such as that of radial flow in an unbounded domain, the mean stress change can be shown to be identically zero (Nowak and McTigue, 1987). Furthermore, it is often appropriate in more general applications to approximate the mean stress as remaining constant. This stipulation reduces Equation (14) to a linear diffusion equation, or a "heat equation," for the pore pressure alone:

$$
C \frac{\partial p}{\partial t}-\frac{k}{\mu} \nabla^{2} p=0
$$

Equation (18) is fundamental to classical hydrology, although it often appears in terms of head rather than pressure. The identity is made by defining the head $h=p / \gamma_{f o} g$, so that Equation (18) becomes

$$
S \frac{\partial h}{\partial t}-K \nabla^{2} h=0
$$

where $S=\gamma_{f 0} g C$ is the specific storativity, and $K=k \gamma_{f 0} g / \mu$ is the hydraulic conductivity. 


\subsection{Anisotropy}

The flow to a circular borehole of finite length and bounded on one end by a plane surface is a two-dimensional problem, even when axisymmetry can be supposed. However, if the permeability is strongly anisotropic, which is often the case in geological materials, the flow can be predominantly in planes normal to the axis of a vertical borehole.

The flow to an open borehole is idealized here as a one-dimensional process (i.e., radial flow occurs in planes normal to the borehole axis). In this configuration, the assumption of isotropy of the hydraulic properties can be viewed as the somewhat less restrictive assumption of transverse isotropy. For example, note that no flow was observed in the two sub-horizontal holes drilled from Room D, while eight vertical holes in the same area produced brine. This might be interpreted as an indication of flow confined predominantly to horizontal planes, perhaps corresponding to compositional or mechanical layering in the rock. This view, however, is not consistent with the observed accumulatic . 1 of brine in the two sub-horizontal holes drilled in Room L. The sub-horizontal holes from Room $D$ were drilled in relatively pure halite, while those from Room L were drilled in argillaceous halite (Finley et al., 1992). 


\subsection{RADIAL FLOW TO AN OPEN BOREHOLE}

\subsection{Pressure Field}

The radial flow model assumes that an open, circular hole is introduced into an unbounded, homogeneous domain at time $t=0$. A definition sketch of the model is shown in Figure 5-1. The initial fluid pressure, $p_{\infty}$, is assumed to be uniform. The open face of the borehole is at atmospheric pressure, $p=0$, causing flow toward the hole, associated with relaxation of the pressure in a zone that grows diffusively outward from the hole. The exact statement of the initialvalue problem is, from Equation (18):

$$
\frac{\partial p}{\partial t}-\frac{c}{r} \frac{\partial}{\partial r}\left(r \frac{\partial p}{\partial r}\right)=0,
$$

with initial condition:

$$
p(r, 0)=p_{\infty},
$$

and boundary conditions:

$$
\begin{gathered}
p(a, t)=0, \\
\lim _{r \rightarrow \infty} p(r, t)=p_{\infty},
\end{gathered}
$$

where $c=k / \mu C$ is the hydraulic diffusivity and $a$ is the radius of the borehole. An analytical solution to Equations (20) through (23) is well known:

$$
\frac{p}{p_{\infty}}=-\frac{2}{\pi} \int_{0}^{\infty} \exp \left(-u^{2} t_{*}\right) f\left(u ; r_{*}\right) \frac{d u}{u},
$$

where

$$
f\left(u ; r_{*}\right)=\frac{J_{0}\left(u r_{*}\right) Y_{0}(u)-Y_{0}\left(u r_{*}\right) J_{0}(u)}{J_{0}^{2}(u)+Y_{0}^{2}(u)}
$$

and where $t_{*}=c t / a^{2}, r_{*}=r / a$, and $J_{0}$ and $Y_{0}$ are Bessel functions of the first and second kind of order zero, respectively (Crank, 1975). 


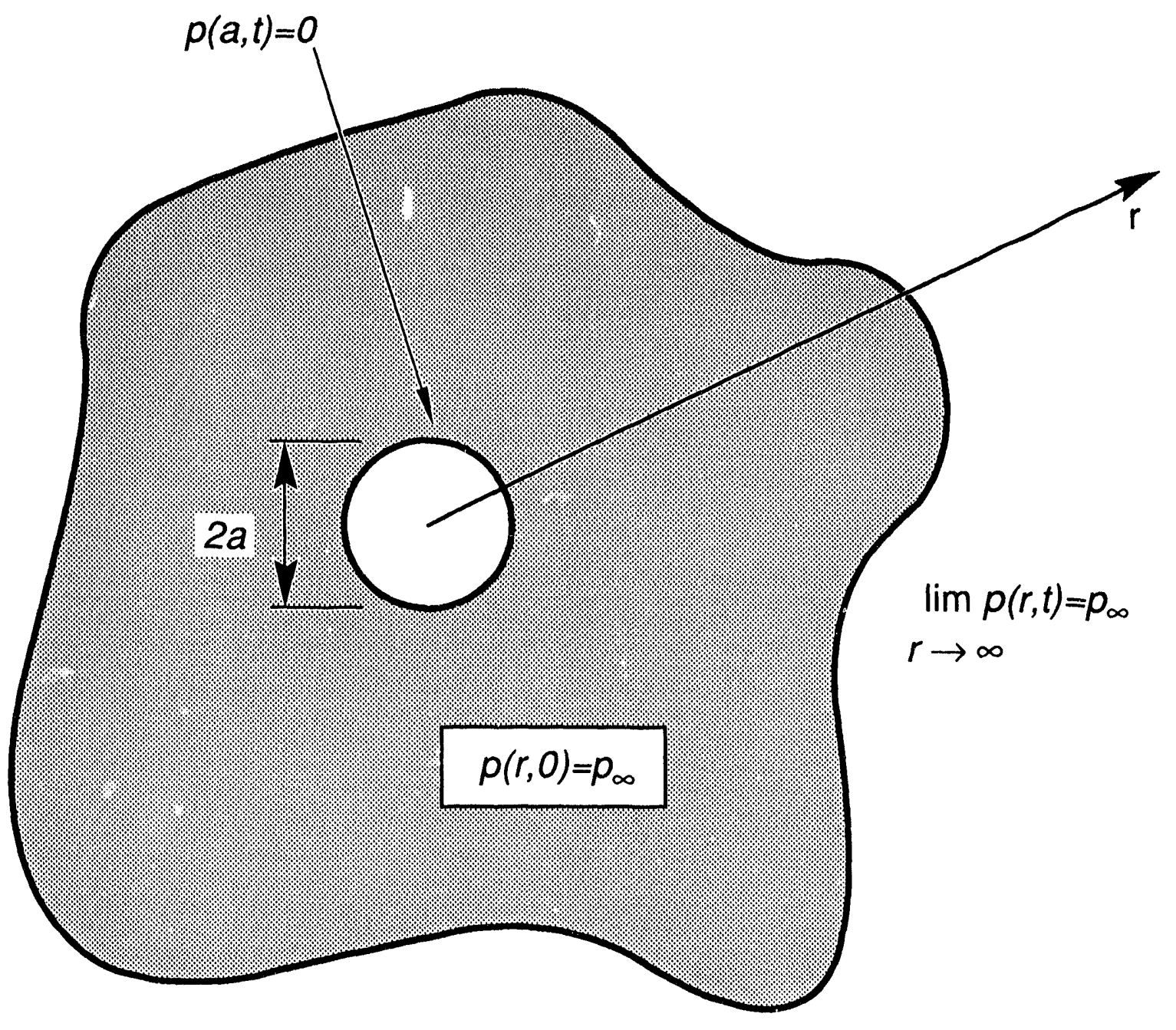

TRI-6119-276-0

Figure 5-1. Definition sketch for radial flow to a borehole. 
Although Equation (24) is a closed-form solution to the problem, it is difficult to evaluate accurately because the integrand is singular at the lower limit of integration. However, the singularity is integrable, so that Equation (24) can be evaluated accurately if appropriate care is taken.

To isolate the singular part of the integrand, one can partition the integral in Equation (24) into two parts:

$$
\frac{p}{p_{\infty}}=-\frac{2}{\pi} \int_{0}^{\varepsilon} \exp \left(-u^{2} t_{*}\right) f\left(u ; r_{*}\right) \frac{d u}{u}-\frac{2}{\pi} \int_{\varepsilon}^{\infty} \exp \left(-u^{2} t_{*}\right) f\left(u ; r_{*}\right) \frac{d u}{u}
$$

where $\varepsilon$ can be chosen to be arbitrarily small. The expansions of $J_{0}(\zeta)$ and $Y_{0}(\zeta)$ for small argument are given by:

$$
\begin{gathered}
J_{0}(\zeta)=1-\frac{\zeta^{2}}{4}+\ldots \\
Y_{11}(\zeta)=\frac{2}{\pi}\left(1-\frac{\zeta^{2}}{4}\right) \ln \left(\frac{\beta \zeta}{2}\right)+\ldots
\end{gathered}
$$

where $\beta=\exp (\gamma)$, and $\gamma=0.5772 \ldots$ is Euler's constant. Substitution of Equations (27) and (28) into the first integral in Equation (26), and expansion about $u=0$ gives, to leading order:

$$
\lim _{u \rightarrow 0}-\frac{2}{\pi} \int_{0}^{\varepsilon} \exp \left(-u^{2} t_{*}\right) f\left(u, r_{*}\right) \frac{d u}{u}=\ln r_{*} \int_{0}^{\varepsilon} \frac{\exp \left(-u^{2} t_{*}\right)}{\ln ^{2}(\beta u / 2)} \frac{d u}{u}+\cdots
$$

The right-hand side of Equation (29) can be integrated by parts to yield

$$
\lim _{u \rightarrow 0}-\frac{2}{\pi} \int_{0}^{\varepsilon} \exp \left(-u^{2} t_{*}\right) f\left(u, r_{*}\right) \frac{d u}{u}=-\frac{\exp \left(-\varepsilon^{2} t_{*}\right) \ln r_{*}}{\ln (\beta \varepsilon / 2)}+O\left(\frac{\varepsilon^{2} t_{*}}{\ln \varepsilon}\right) .
$$

Thus, Equation (24) can be evaluated accurately by separating the singular part of the integrand, integrating it analytically, and evaluating the remainder by numerical quadrature. Substitution of Equation (30) into Equation (26) gives the final form used:

$$
\frac{p}{p_{\infty}} \simeq-\frac{\exp \left(-\varepsilon^{2} t_{*}\right) \ln r_{*}}{\ln (\beta \varepsilon / 2)}-\frac{2}{\pi} \int_{\varepsilon}^{\infty} \exp \left(-u^{2} t_{*}\right) f\left(u ; r_{*}\right) \frac{d u}{u} .
$$


Evaluations of Equation (31) have been carried out, setting $\varepsilon=10^{-8}$, and performing the integral by the Gauss quadrature routine DGAUS8 in the SLATEC subroutine package. The error tolerance in the numerical integrator was set to $E R R=10^{-6}$, and the calculations were carried out on a VAX 8650 using double-precision arithmetic. Figure 5-2 shows Equation (31) evaluated for $1 \leq r_{*} \leq 5$ and $t_{*}=0.01,0.1,0.5,1.0,5.0,10.0,50.0$. The results shown here differ slightly from those reported by Nowak and McTigue (1986) and Nowak, McTigue, and Beraun (1988) because of the more careful treatment of the integral in Equation (24) near $u=0$. The most significant differences are evident at later times and larger radial coordinates, where the pressures are typically somewhat larger than in the original calculations. Thus, the numerical quadrature applied directly to Equation (24) appears to underestimate the contribution near the singularity.

The exact numerical values on which Figure 5-2 is based are reproduced in Table 5-1 for reference. Note that the integration scheme appears to have difficulty resolving values asymptotically close to unity. For example, at $t_{*}=0.01$, the dimensionless pressure reaches a maximum value of 0.9986 at $r_{*}=1.6$, and decreases monotonically to 0.9952 at $r_{*}=5.0$, rather than continuing to approach 1.0. No attempt was made to resolve this problem for the calculations shown in Table 5-1, as it appears to have little practical consequence. However, for reference, the asymptotic expansion of Equation (24) for large $X=\left(r_{*}-1\right) / 2 \sqrt{t_{*}}$, which can be used to obtain a more accurate evaluation of the pressure far from the hole, is given by:

$$
\lim _{X \rightarrow \infty} \frac{p}{p_{\infty}}=1-\frac{1}{\sqrt{\pi r_{*}}} X^{-1} \exp \left(-X^{2}\right)+\cdots \text {. }
$$

\subsection{Fluid Flux at the Borehole}

A quantity that can be estimated from field data is the flux into the hole, $q_{r}(a, t)$, which can be obtained from Equation (24) by application of Darcy's law:

$$
q_{r}(a, t)=-\frac{k}{\mu} \frac{\partial p}{\partial r}(a, t)
$$

where $k$ is the permeability and $\mu$ is the brine viscosity. The resulting expression has been noted previously by Nowak and McTigue (1987) and Nowak et al. (1988):

$$
q_{*}=\frac{4}{\pi^{2}} \int_{0}^{\infty} \frac{\exp \left(-u^{2} t_{*}\right)}{J_{0}^{2}(u)+Y_{0}^{2}(u)} \frac{d u}{u},
$$




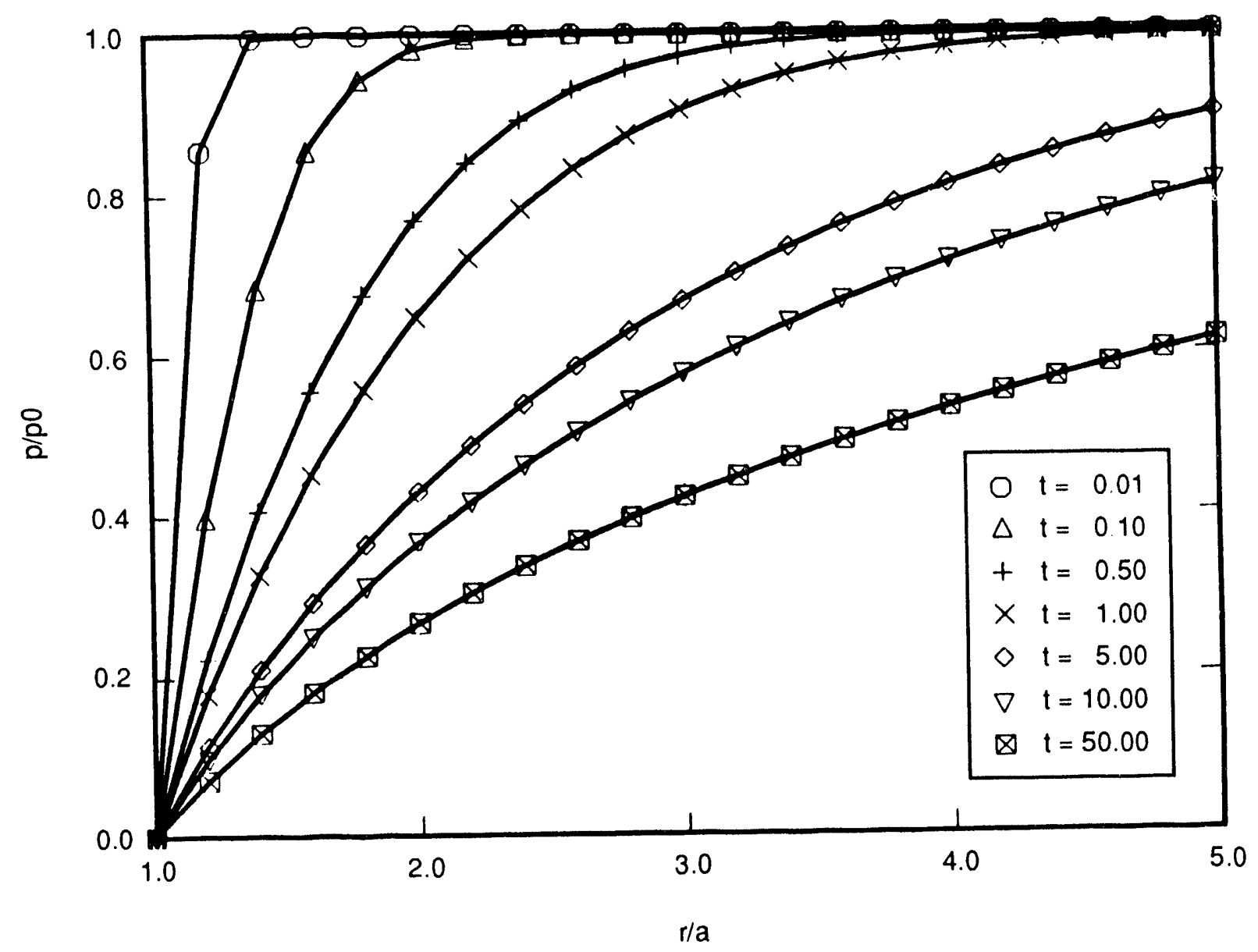

TRI.6119-277.0

Figure 5-2. Pore pressure profiles at various times based on Equation (31); radial coordinate, $r$, is normalized by borehole radius, $a$; pressure, $p$, is normalized by initial value $p_{\infty}$. 
Table 5-1. Pressure Profiles at Various Times Based on Equation (31)

\begin{tabular}{|c|c|c|c|c|c|c|c|}
\hline \hline$r_{*}$ & $t *=0.01$ & $t *=0.1$ & $t *=0.5$ & $t *=1.0$ & $t *=5.0$ & $t *=10.0$ & $t *=50.0$ \\
\hline 1.0 & 0.0000 & 0.0000 & 0.0000 & 0.0000 & 0.0000 & 0.0000 & 0.0000 \\
1.2 & 0.8557 & 0.3987 & 0.2234 & 0.1785 & 0.1139 & 0.0968 & 0.0702 \\
1.4 & 0.9950 & 0.6830 & 0.4067 & 0.3273 & 0.2101 & 0.1785 & 0.1296 \\
1.6 & 0.9986 & 0.8553 & 0.5558 & 0.4525 & 0.2930 & 0.2492 & 0.1810 \\
1.8 & 0.9982 & 0.9428 & 0.6749 & 0.5580 & 0.3656 & 0.3113 & 0.2263 \\
2.0 & 0.9979 & 0.9798 & 0.7679 & 0.6466 & 0.4298 & 0.3666 & 0.2668 \\
2.2 & 0.9977 & 0.9927 & 0.8384 & 0.7203 & 0.4871 & 0.4164 & 0.3035 \\
2.4 & 0.9974 & 0.9962 & 0.8904 & 0.7812 & 0.5386 & 0.4614 & 0.3368 \\
2.6 & 0.9972 & 0.9969 & 0.9274 & 0.8308 & 0.5850 & 0.5025 & 0.3675 \\
2.8 & 0.9969 & 0.9969 & 0.9529 & 0.8706 & 0.6270 & 0.5401 & 0.3959 \\
3.0 & 0.9967 & 0.9967 & 0.9698 & 0.9022 & 0.6650 & 0.5747 & 0.4222 \\
3.2 & 0.9965 & 0.9965 & 0.9806 & 0.9269 & 0.6994 & 0.6066 & 0.4468 \\
3.4 & 0.9963 & 0.9963 & 0.9872 & 0.9458 & 0.7306 & 0.6360 & 0.4698 \\
3.6 & 0.9962 & 0.9961 & 0.9912 & 0.9600 & 0.7590 & 0.6633 & 0.4914 \\
3.8 & 0.9960 & 0.9960 & 0.9934 & 0.9706 & 0.7847 & 0.6886 & 0.5118 \\
4.0 & 0.9959 & 0.9958 & 0.9945 & 0.9783 & 0.8079 & 0.7121 & 0.5311 \\
4.2 & 0.9957 & 0.9957 & 0.9951 & 0.9838 & 0.8289 & 0.7340 & 0.5494 \\
4.4 & 0.9956 & 0.9955 & 0.9953 & 0.9876 & 0.8479 & 0.7542 & 0.5668 \\
4.6 & 0.9954 & 0.9954 & 0.9953 & 0.9902 & 0.8650 & 0.7731 & 0.5833 \\
4.8 & 0.9953 & 0.9953 & 0.9953 & 0.9919 & 0.8804 & 0.7906 & 0.5990 \\
\hline \hline & 0.9952 & 0.9951 & 0.9952 & 0.9931 & 0.8942 & 0.8069 & 0.6140 \\
\hline
\end{tabular}

* Radial coordinate, $r$, is normalized by borehole radius, $a$; time, $t$, is normalized by $a^{2} / c$; pressure, $p$, is normalized by initial value, $p_{\infty}$. 
where $q_{*}$ is the magnitude of the normalized flux at the borehole, $q_{*}=\left|q_{r}\right|(a, t) / q_{0}=-q_{r}(a, t) / q_{0}$, and the scale of the flux, $q_{0}$, is given by $q_{0}=k p_{\infty} / \mu a$.

Equation (34) encounters the same difficulty discussed above in the context of the pressure profiles: the integrand is singular at $u=0$, and numerical quadrature routines cannot easily resolve this. However, the singularity is of the same form as that in Equation (24), and is therefore integrable. The identical procedure can be applied to Equation (34). However, the same result can be achieved by differentiating Equation (31) directly, giving:

$$
q_{*} \simeq-\frac{\exp \left(-\varepsilon^{2} t_{*}\right)}{\ln (\beta \varepsilon / 2)}+\frac{4}{\pi^{2}} \int_{\varepsilon}^{\infty} \frac{\exp \left(-u^{2} t_{*}\right)}{J_{0}^{2}(u)+Y_{0}^{2}(u)}, \frac{d u}{u} .
$$

Evaluations of Equation (35) have been carried out in the same fashion as those of Equation (31) described above, again taking $\varepsilon=10^{-8}$, and ERR $=10^{-6}$. The results are shown in Table 5-2 and in Figure 5-3. The fluxes computed here are slightly higher than those obtained by Nowak and McTigue (1986) and Nowak, McTigue, and Beraun (1988), again because numerical quadrature applied directly to Equation (34) underestimates the contribution near the singularity.

A check against independent calculations is possible for $t_{*}<1.0$, based on results published by Jaeger and Clarke (1942-43). The fluxes reported in Table 5-2 for $t_{*}$ from 0.01 to 0.8 are identical to the vaiues given by Jaeger and Clarke to within the three decimal places and reproduced in Table 5-2. Jaeger and Clarke do not report explicit results for $t_{*}>0.99$.

\subsection{Cumulative Volume}

The cumulative volume of fluid per unit area of borehole wall, $v$, is obtained by integrating Equation (35) over time:

$$
\nu_{*} \simeq-\frac{t_{*}}{\ln (\beta \varepsilon / 2)}+\frac{4}{\pi^{2}} \int_{\varepsilon}^{\infty} \frac{\left[1-\exp \left(-u^{2} t_{*}\right)\right]}{J_{0}^{2}(u)+Y_{n}^{2}(u)} \frac{d u}{u^{3}}+O\left(\frac{\varepsilon^{2} t_{*}^{2}}{\ln \varepsilon}\right),
$$

where $\nu_{*}=\nu / v_{0}$, and $v_{0}=k p_{\infty} a / \mu c=C p_{\infty} a$ is the reference volume scale. 
Table 5-2. Fluid flux at a borehole*

\begin{tabular}{|c|c|c|}
\hline \hline & \multicolumn{2}{|c|}{$q^{*}$} \\
\hline & Equation (35) & J\&C (1942) \\
\hline $0.1 \mathrm{E}-01$ & 6.1291 & 6.129 \\
$0.2 \mathrm{E}-01$ & 4.4718 & 4.472 \\
$0.4 \mathrm{E}-01$ & 3.2969 & 3.297 \\
$0.6 \mathrm{E}-01$ & 2.7748 & 2.775 \\
$0.8 \mathrm{E}-01$ & 2.4625 & 2.462 \\
$0.1 \mathrm{E}+00$ & 2.2489 & 2.249 \\
$0.2 \mathrm{E}+00$ & 1.7154 & 1.715 \\
$0.4 \mathrm{E}+00$ & 1.3326 & 1.333 \\
$0.6 \mathrm{E}+00$ & 1.1601 & 1.160 \\
$0.8 \mathrm{E}+00$ & 1.0559 & 1.056 \\
$0.1 \mathrm{E}+01$ & 0.9839 & \\
$0.2 \mathrm{E}+01$ & 0.8007 & \\
$0.4 \mathrm{E}+01$ & 0.6645 & \\
$0.6 \mathrm{E}+01$ & 0.6010 & \\
$0.8 \mathrm{E}+01$ & 0.5617 & \\
$0.1 \mathrm{E}+02$ & 0.5340 & \\
$0.2 \mathrm{E}+02$ & 0.4613 & \\
$0.4 \mathrm{E}+02$ & 0.4041 & \\
$0.6 \mathrm{E}+02$ & 0.3762 & \\
$0.8 \mathrm{E}+02$ & 0.3584 & \\
$0.1 \mathrm{E}+03$ & 0.3457 & \\
$0.2 \mathrm{E}+03$ & 0.3109 & \\
$0.4 \mathrm{E}+03$ & 0.2822 & \\
$0.6 \mathrm{E}+03$ & 0.2676 & \\
$0.8 \mathrm{E}+03$ & 0.2580 & \\
$0.1 \mathrm{E}+04$ & 0.2511 & \\
$0.2 \mathrm{E}+04$ & 0.2316 & \\
$0.4 \mathrm{E}+04$ & 0.2149 & \\
$0.6 \mathrm{E}+04$ & 0.2061 & \\
$0.8 \mathrm{E}+04$ & 0.2003 & \\
$0.1 \mathrm{E}+05$ & 0.1961 & \\
\hline \hline & & \\
\hline
\end{tabular}

*J\&C = Jaeger and Clarke 


\subsection{Late-Time, Asymptotic Flux}

The asymptotic expansion of Equation (34) for $t_{*} \gg 1$ takes a particularly simple form useful for fitting data. The development is reproduced here in detail.

In order to find the late-time, asymptotic expansion of the fluid flux (Equation 34), let $\eta^{2}=u^{2} t_{*}$, so that large $t_{*}$ corresponds to small arguments of the Bessel functions. Introduce the appropriate expansions for small argument from Equations (27) and (28), and expand the integrand:

$$
\begin{aligned}
\lim _{t \rightarrow \infty} q_{*}= & \int_{0}^{\infty} \frac{\exp \left(-\eta^{2}\right)}{\left[\ln \left(\beta \eta / 2 t_{*}^{1 / 2}\right)\right]^{2}}\left\{1-\frac{\pi^{2} / 4}{\left[\ln \left(\beta \eta / 2 t_{*}^{1 / 2}\right)\right]^{2}}+\cdots\right\} \frac{d \eta}{\eta} \\
& +\frac{1}{2 t_{*}} \int_{0}^{\infty} \frac{\eta \exp \left(-\eta^{2}\right)}{\left[\ln \left(\beta \eta / 2 t_{*}^{1 / 2}\right)\right]^{2}} d \eta+\cdots
\end{aligned}
$$

where $\beta=\exp (\gamma)$, and $\gamma=0.5772 \ldots$ is Euler's constant. Integration of each term in Equation (37) by parts yields:

$$
\begin{aligned}
\lim _{t \rightarrow \infty} q_{*}= & -2 \int_{0}^{\infty} \frac{\eta \exp \left(-\eta^{2}\right)}{\ln \left(\beta \eta / 2 t_{*}^{1 / 2}\right)}\left[1-\frac{\pi^{2}}{6}\left(\eta^{4}-3 \eta^{2}+1\right)+\cdots\right] d \eta \\
& -\frac{1}{t_{*}} \int_{0}^{\infty} \frac{\eta\left(\eta^{2}-1\right) \exp \left(-\eta^{2}\right)}{\ln \left(\beta \eta / 2 t_{*}^{1 / 2}\right)} d \eta+\cdots
\end{aligned}
$$

Expansion of $1 / \ln \left(\beta \eta / 2 t_{*}^{1 / 2}\right)$ in powers of $1 / \ln \left(4 t_{*} / \beta^{2}\right)$ gives:

$$
\frac{1}{\ln \left(\beta \eta / 2 t_{*}^{1 / 2}\right)}=-\frac{2}{\ln \left(4 t_{*} / \beta^{2}\right)}\left\{1+\frac{2 \ln \eta}{\ln \left(4 t_{*} / \beta^{2}\right)}+\frac{4(\ln \eta)^{2}}{\left[\ln \left(4 t_{*} / \beta^{2}\right)\right]^{2}}+\cdots\right\}
$$

Substitution of Equation (39) into Equation (38) and integration term-by-term yields: 


$$
\begin{aligned}
\lim _{t \rightarrow \infty} q_{*} & =\frac{2}{\ln \left(4 t_{*} / \beta^{2}\right)}\left[1-\frac{\gamma}{\left[\ln \left(4 t_{*} / \beta^{2}\right)\right]}+\frac{\left(\frac{\pi^{2}}{6}+\gamma^{2}\right)}{\left[\ln \left(4 t_{*} / \beta^{2}\right)\right]^{2}}+\cdots\right] \\
& -\frac{2 \pi^{2} / 3}{\ln \left(4 t_{*} / \beta^{2}\right)}\left[0+0+\frac{1}{\left[\ln \left(4 t_{*} / \beta^{2}\right)\right]^{2}}+\cdots\right] \\
& +\frac{2 / t_{*}}{\ln \left(4 t_{*} / \beta^{2}\right)}\left[0+\frac{1 / 2}{\ln \left(4 t_{*} / \beta^{2}\right)}+\cdots\right] .
\end{aligned}
$$

Combination of the first four nonzero terms in Equation (40), and neglect of the smaller term of order $t_{*}^{-1} \ln ^{-2}\left(t_{*}\right)$, yields the final result:

$$
\lim _{t \rightarrow \infty} q_{*}=\frac{2}{\ln \left(4 t_{*} / \beta^{2}\right)}\left[1-\frac{\gamma}{\ln \left(4 t_{*} / \beta^{2}\right)}-\frac{\left(\frac{\pi^{2}}{6}-\gamma^{2}\right)}{\left[\ln \left(4 t_{*} / \beta^{2}\right)\right]^{2}}+\cdots\right] .
$$

Figure 5-4 shows an evaluation of the full integral solution for the flux from Equation (35), along with the late-time approximations based on Equation (41). It is evident by inspection of Figure 5-4 that one or two terms of the series given by Equation (41) capture the general trend of the exact solution at late time, but overestimate the flux somewhat. The two-term expansion yields a flux that is $5 \%$ too high at $t_{*}=100$ and $3 \%$ too high at $t_{*}=1000$. The three-term expansion is very close (less than $2 \%$ error) for $t_{*}>100$. Table 5-3 presents the late-time approximations compared to the "exact" fluid-flux solution.

A simple scheme for fitting data to extract hydraulic properties is suggested by taking the inverse of Equation (41):

$$
\lim _{t \rightarrow \infty} q_{*}^{-1}=\frac{1}{2} \ln \left(\frac{4 t_{*}}{\beta^{2}}\right)\left\{1+\frac{\gamma}{\ln \left(4 t_{*} / \beta^{2}\right)}+\frac{\pi^{2} / 6}{\left[\ln \left(4 t_{*} / \beta^{2}\right)\right]^{2}}+\cdots\right\}
$$




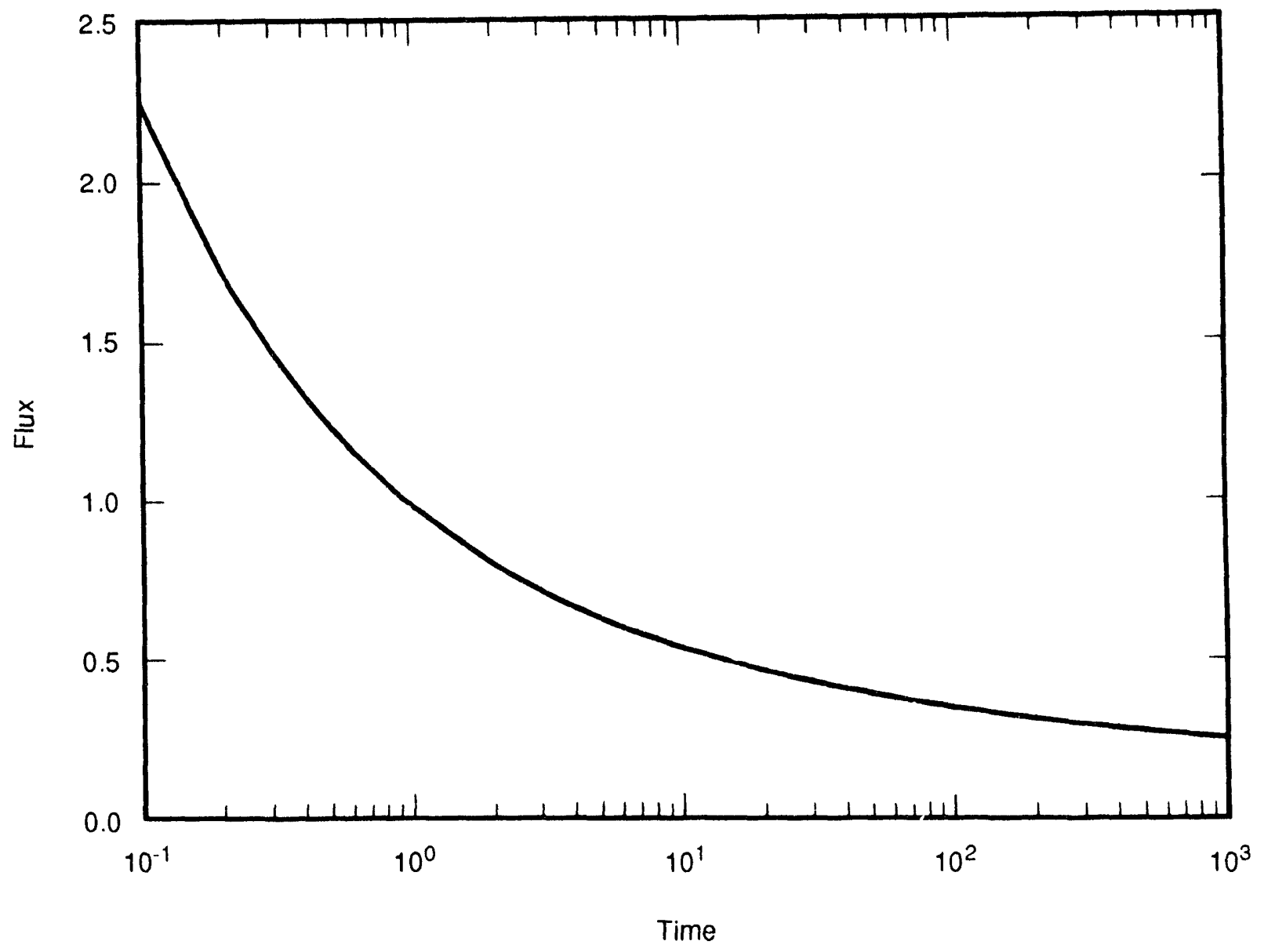

TR1.6119-278.0

Figure 5-3. Fluid flux at a borehole, evaluated from "exact" solution, Equation (35). 


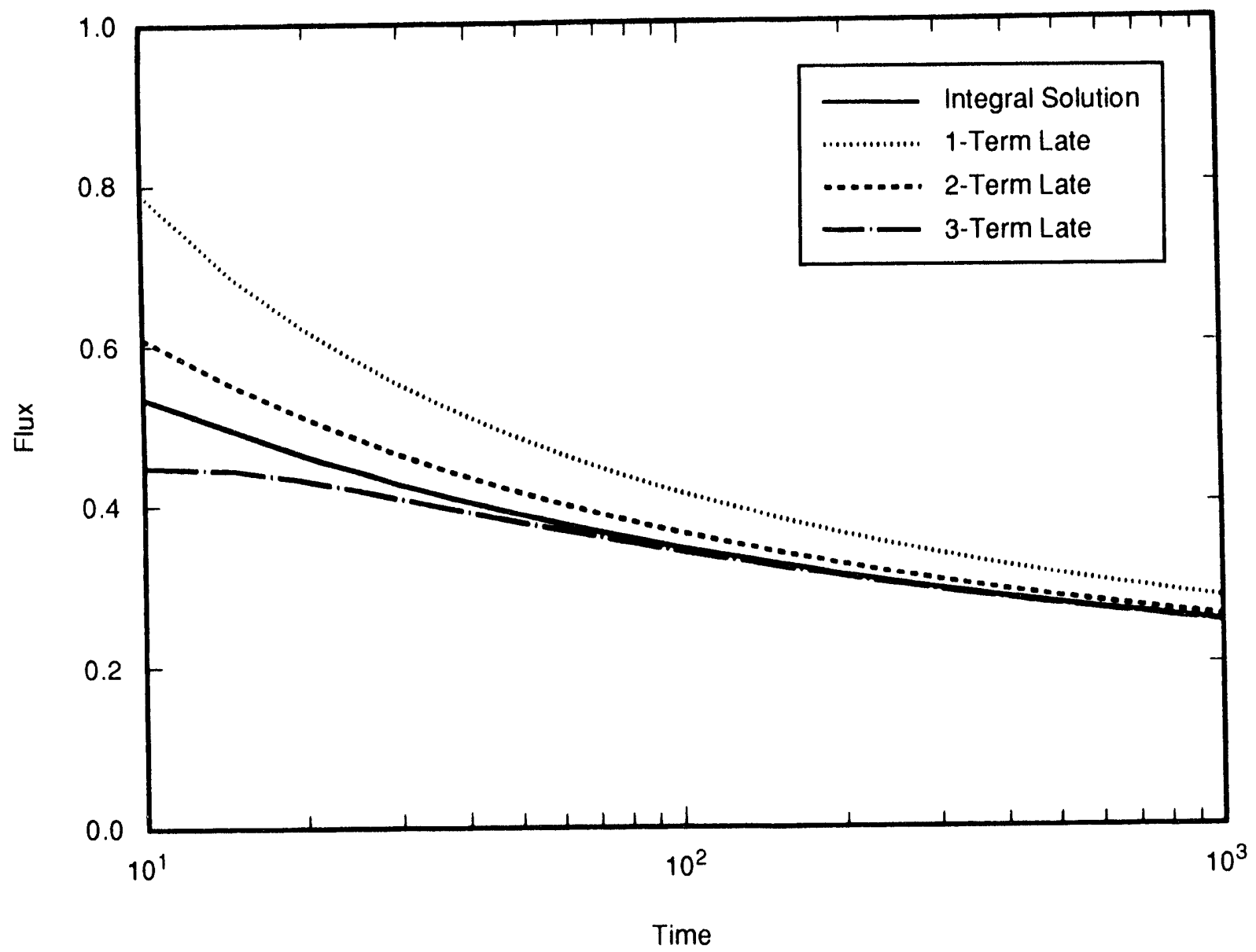

TRI-6119-279-0

Figure 5-4. Comparison of late-time approximations (Equation 41) and "exact" solution (Equation 35) for fluid flux at a borehole; time, $t$, is normalized by characteristic diffusion time, $a^{2} / c$; flux, $\left|q_{r}\right|(a, t)$, is normalized by $q_{0}=k p_{\infty} / \mu a$. 
Table 5-3. Comparison of Late-time Approximations and "Exact" Solution for Fluid Flux at a Borehole

\begin{tabular}{|r|c|c|c|c|}
\hline \hline \multirow{2}{*}{$t_{*}$} & \multicolumn{3}{|c|}{$q_{*}$} \\
\cline { 2 - 4 } & \multicolumn{3}{|c|}{ Late-time Approx. } & \multirow{2}{*}{ "Exact" } \\
\cline { 2 - 4 } & 1 Term & 2 Terms & 3 Terms & \\
\hline 10 & 0.7891 & 0.6094 & 0.4483 & 0.5340 \\
50 & 0.4826 & 0.4154 & 0.3785 & 0.3883 \\
100 & 0.4135 & 0.3641 & 0.3410 & 0.3457 \\
500 & 0.3102 & 0.2825 & 0.2727 & 0.2739 \\
1000 & 0.2801 & 0.2575 & 0.2503 & 0.2511 \\
5000 & 0.2286 & 0.2135 & 0.2096 & 0.2100 \\
10000 & 0.2118 & 0.1989 & 0.1957 & 0.1961 \\
\hline \hline
\end{tabular}

Retaining the first two terms in Equation (42) (i.e., neglecting the term of $O\left(\ln ^{-1}\left(4 t_{*} / \beta^{2}\right)\right)$, and returning to dimensional variables, this takes the convenient form:

$$
\lim _{t * \rightarrow \infty}\left|q_{r}\right|^{-1}(a, t)=A \ln t+B
$$

where

$$
A=\frac{\mu a}{2 k p_{\infty}}
$$

and

$$
B=A \ln \left(\frac{4 c}{a^{2} \beta}\right) .
$$

Thus, a plot of the inverse flux at the borehole versus $\ln t$ is approximately linear at sufficiently late time. The slope, $A$, is an indicator of the permeability, and the intercept, $B$, is an indicator of the hydraulic diffusivity through the simple relations:

$$
k=\frac{\mu a}{2 p_{\infty} A},
$$

and

$$
c=\frac{a^{2} \beta}{4} \exp \left(\frac{B}{A}\right) \quad(\text { two }- \text { term expansion })
$$


Note that, if only the first term of the expansion given in Equation (42) is retained, Equation (47) is replaced by

$$
c=\frac{a^{2} \beta^{2}}{4} \exp \left(\frac{B}{A}\right) \text { (leading order). }
$$

Thus, the higher-order approximation simply shifts the curve upward by a constant, $A \gamma$. As a result, a fit based on only the first term in Equation (42) will yield an estimate for the diffusivity (Equation 48) that is a factor of $\beta \simeq 1.78$ times greater than that obtained in view of the two-term expansion (Equation 47).

Figure 5-5 shows a plot of inverse flux versus the logarithm of time for $t_{*}>10$. The open symbols are computed from evaluations of the exact, integral solution. The lines show one, two, and three terms of Equation (42). As noted above, both the leading-order approximation and the next, higher-order approximation are linear on this plot, with the latter simply shifted upward by a constant. Equation (47) will give a better estimate of the hydraulic diffusivity than will Equation (48), but Equation (47) still tends to overestimate $c$. A nonlinear fit of the three-term series given in Equation (42) would give a much better result, and, although more involved than a linear regression based on the first two terms, is still far easier to perform than a fit requiring numerical quadrature on Equation (35).

The inverse-flux fitting method was tested using synthetic data generated by numerical simulations of radial flow (Webb, 1992). Although the permeabilities used in the simulations were recovered with very good accuracy (typically within a few percent), the diffusivities estimated using Equation (47) were typically overestimated by factors of about 50\%. This difficulty is inherent in the method because extrapolation of the late-time fit back to $\ln t=0$ introduces significant error (Jaeger, 1958). 


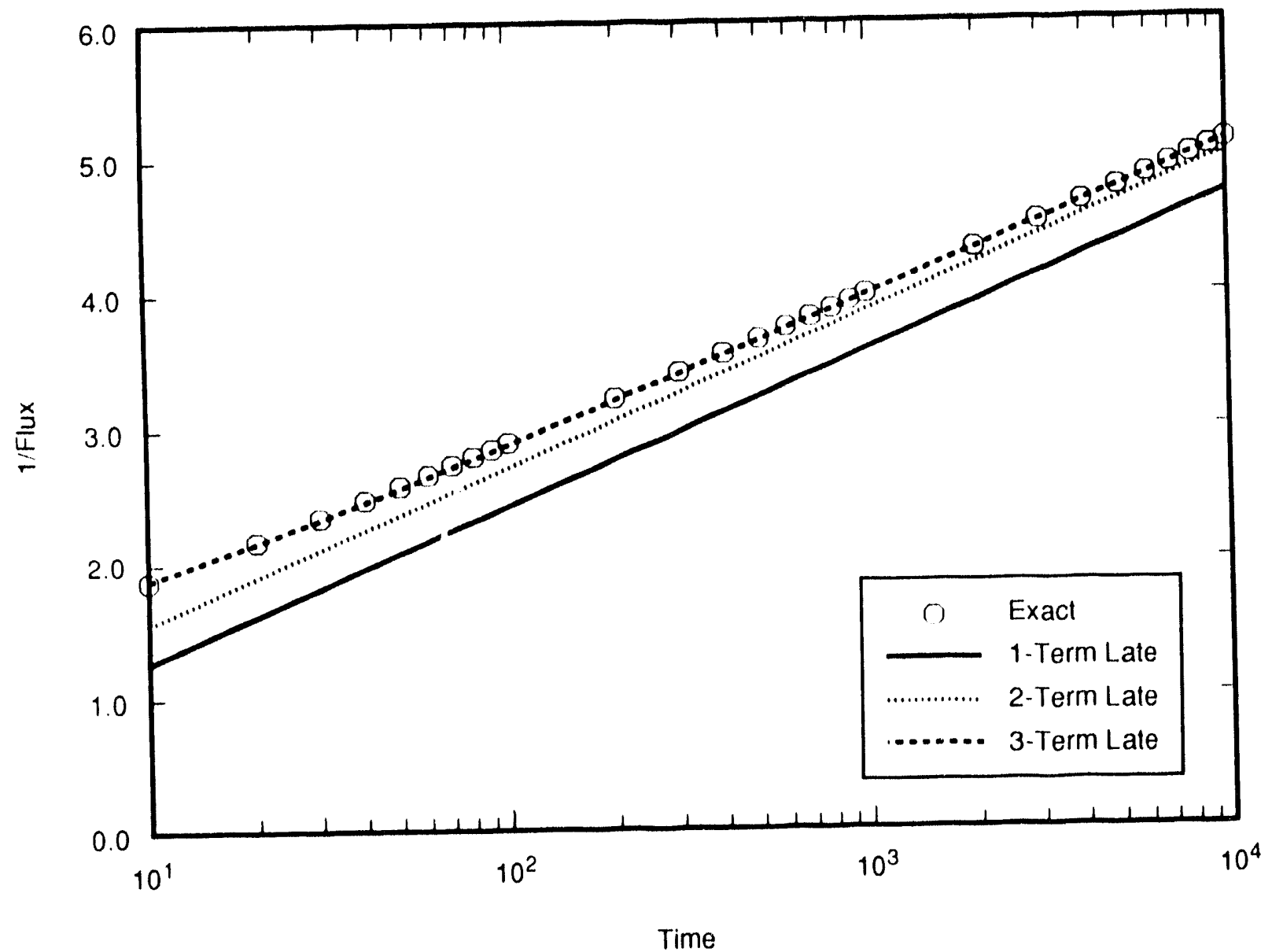

Tค1.6119.280.0

Figure 5-5. Comparison of "exact" and late-time, asymptotic solutions for inverse of borehole flux; time, $t$, is normalized by chatracteristic diffusion time, $a^{2} / c ;$ flux, $\left|q_{r}\right|(a, t)$, is normalized by $q_{01}=k p p_{\infty} / \mu a$. 
A good estimate of the diffusivity from late-time data is difficult to obtain if other processes affect the flow at early time. For example, suppose that the early-time flow is delayed by the growth of microcracks, dilatation, and accompanying "storage" of brine. After some time, the flow may behave in the classical fashion, and the slope of the $|q|^{-1}$ versus $\ln t$ plot would reflect the permeability. However, the time should be "re-zeroed" to account for the delay. Without some rational basis for such a correction, the diffusivity inferred by this method is inherently unreliable if processes not accounted for are significant. An example that has been studied in the context of the thermal-conductivity probe is the effect of contact resistance between the probe and the medium (Blackwell, 1954).

\subsection{Flow Limited to Discrete Horizons}

Consistent with the analytical model discussed above, the present reduction of data assumes that the hole penetrates a domain of isotropic, homogeneous salt. Thus, production of brine is assumed to be uniform along its length. Because the brine collection method integrates over the entire borehole length, a more detailed breakdown of the distribution of flow cannot be extracted. However, if one were to assume that all of the accumulated brine in a particular hole came from some discrete fraction of its total length, $L_{p} / L$, where $L_{p}$ is the producing length and $L$ is the total length, then the parameters estimated here could be rescaled accordingly. In particular, both the inferred permeability and capacitance would be multiplied by $\left(L_{p} / L\right)^{-1}$ to obtain the appropriate parameters for the producing layer, with the remainder of the stratigraphy regarded as totally impermeable. 


\subsection{PARAMETER ESTIMATION}

\subsection{Method}

The fits reported here for the full-flux and cumulative-volume histories were performed with the parameter-estimation code ESTIM (Hills, 1987). The code seeks the set of model parameters that minimizes the sum of squares of residuals, or differences between observed and simulated quantities. In the present case, the data used are either brine flux or cumulative brine volume, estimated as discussed in Section 3. The simulations are numerical evaluations of the exact solutions for radial seepage to a long hole, given by Equations (35) and (36) for flux and volume, respectively.

Programs that evaluate the functions describing seepage to a borehole, Equations (35) and (36), are called as subroutines by ESTIM. These function evaluations were thoroughly tested as noted in Section 5.2. Each of the analytical functions is a two-parameter description of the flow; one parameter scales the magnitude of the flux or volume, and the other is a characteristic time over which the flow evolves. Thus, Equation (35) can be fit to data for brine flux by seeking the magnitude scale, $k p_{\infty} / \mu a$, and the time scale, $a^{2} / c$. The brine viscosity, $\mu$, and borehole radius, $a$, can be determined by independent measurements with some confidence, leaving the product of the permeability and the initial pressure, $k p_{\infty}$, and the diffusivity, $c$, as unknowns. A further assumption for the initial pore pressure $p_{\infty}$ isolates the permeability. The capacitance is then easily calculated from $C=k / \mu c$.

Note that, with few exceptions, the observed flow rates fall in a reasonably narrow range. Thus, the product of permeability and initial pressure is fairly well constrained in most cases considered here. However, the detailed history of the flux for many holes was somewhat erratic, so fits for the diffusivity are more uncertain.

ESTIM employs a local least-squares method (i.c., the code seeks a local minimum in the sum of squares of residuals as a function of the unknown parameters). In general, experience with the present data suggests that the minimum local to a reasonable initial estimate of the parameters is the desired global minimum. ESTIM requires as input a set of initial estimates for the parameters to be determined, as well as upper and lower bounds on their possible values. At each iteration, the code constructs a sensitivity matrix consisting of the partial derivatives of the error measure (the sum of squares of residuals) with respect to the unknown parameters. These derivatives were 
approximated for all calculations reported here by first-order differences. ESTIM then employs a quadratic least-squares method to optimize the parameter estimates.

The parameter estimation code includes an option to perform a propagation of variance analysis. This option was exercised for the fits to the full flux histories reported here; it was not used for the cumulative-volume fits. The analysis provides a measure of the sensitivity of the estimated parameters to small random errors in the data. Note that the standard deviations returned by ESTIM are "... estimates of the true standard deviations of the estimated parameters [if]

1. ESTIM converges to the global minimum.

2. The simulator models the physics of the problem well.

3. The property models are appropriate for the materials in question.

4. The measurements errors are random, independent, [and] have zero mean, with either a uniform standard deviation ... or known and specified standard deviation" (Hills, 1987).

For the propagation of variance performed on the analysis of the full flux histories, the measurement error is assumed uniform for every sampling interval in each borehole among the entire group. In particular, the standard deviation of the measurements of mass of brine collected was assumed to be $\pm 5 \mathrm{~g}$ for each sample taken. For a uniform, seven-day sample interval, this corresponds to a measurement error of $\pm 0.71 \mathrm{~g} / \mathrm{d}$ on the total mass flow rate, which is comparable to the error estimates reported by Finley et al. (1992), based on laboratory repeatability tests and on a detailed statistical analysis of the field data by Rutherford (1992). This estimate for the error in the measured mass flow rate was then divided by the brine density and the borehole wall area for each hole to yield an estimate of the error in the flux. ESTIM returns normalized standard deviations for each parameter, which are then multiplied by the corresponding error estimate for the raw data to yield the standard deviation associated with each parameter in a given fit. These values are reported in Section 6.2.2.

\subsection{Fits Based on the Full Flux Histories}

The principal emphasis in this report is on fits based on the full history of the brine flux for each borehole. Fits to the flux are believed to yield the most significant information with regard to the hydraulic diffusivity. The least-squares fitting routine tends to place greater weight on the larger numerical values in a given data set. Because the flux is maximum at early time, fits to the 
flux data tend to weight the early-time response. The flow changes most rapidly at early time, so that the most important information with regard to the evolution of the flow through time, which is characterized by the diffusivity, is contained in the early-time data.

One disadvantage of fitting the flux is that the data reduction entails an approximation to obtain flow rates. In particular, the field sampling program yields the cumulative volume over the sampling interval. In the present case, the mean flow rate over the sampling interval (an exact quantity) was calculated and assigned to a time corresponding to the midpoint of the interval (an approximation). This approximation is valid when the rate of change of flow rate as well as the sampling interval are relatively small. A second disadvantage to fitting the flux is that the data often exhibit considerable scatter (i.e., while the cumulative volume must always be a nondecreasing sequence of data and often is reasonably smooth, the discrete approximations to its derivative can be highly variable). The least-squares routine can encounter difficulty fitting data with large scatter.

\subsubsection{Deleted Data and Other Data Reduction}

It is often necessary to discard extreme outliers in order to obtain a good fit by least-squares methods. An effort was made in this study to delete a minimum of data.

For many of the drillholes, no brine was obtained in the first sampling, yielding an estimated flux of zero at the earliest available time. This datum was discarded in every case in which it arose. Because the model (Equation 34) predicts large flux at early time ( $q \rightarrow \infty$ as $t \rightarrow 0)$, the residual between a computed value and a zero datum is large, and the zero is given great weight. Thus, the optimization scheme seeks to make the diffusivity very large, and does not converge.

Deleted data, as well as other data reduction applied in special cases, are summarized below for the fits to the full flux histories; data are listed as pairs in the format (time, flux), with time in seconds and flux in meters per second:

- DBT10 - deleted initial zero $\left(2.56 \times 10^{5}, 0.0\right)$ and one outlier $\left(4.49 \times 10^{7}, 7.88 \times 10^{-11}\right)$

- DBT11 - deleted initial zero $\left(4.01 \times 10^{4}, 0.0\right)$

- DBT12 - deleted initial zero $\left(8.33 \times 10^{4}, 0.0\right)$

- DBT13 - no data deleted

- DBT14A - deleted all data for increasing flux, $t>1.26 \times 10^{7} \mathrm{~s}(\sim 146$ days $)$ 
- DBT14B - deleted initial zero $\left(3.03 \times 10^{5}, 0.0\right)$ and all data for increasing flux, $t>1.54 \times 10^{7} \mathrm{~s}(\sim 178$ days); all flow for this time interval was assumed to be to the newly extended section of the borehole $(\mathrm{L}=\sim 3.0 \mathrm{~m})$

- DBT15A - no data deleted (through $t=2.44 \times 10^{7} \mathrm{~s} \simeq 285 \mathrm{~d}$ )

- DBT15B - deleted first three data (increasing flux): $\left(3.04 \times 10^{5}, 3.02 \times 10^{-12}\right)$, $\left(9.06 \times 10^{5}, 1.37 \times 10^{-10}\right),\left(1.51 \times 10^{6}, 1.69 \times 10^{-10}\right)$; volume due to flow to original borehole was calculated from the fit to DBT15A and subtracted from the raw data for DBT15B; remaining volume was assumed to be due to flow to the newly extended section of borehole $(L=\sim 3.0 \mathrm{~m})$

- DBT31A - deleted first datum (increasing flux): $\left(6.88 \times 10^{5}, 1.06 \times 10^{-10}\right)$; deleted all data for erratic flow $t>1.32 \times 10^{7} \mathrm{~s}(\sim 152$ days $)$

- DBT31B - no fit obtained; increasing flow rate

- DBT31C - fit only to period of declining flux from 196 to 335 days (referenced to time of enlargement at 253 days from initial drilling for DBT31A); deleted 27 zero-flux values from time of enlargement to time of first measured brine accumulation; deleted all data for $t>2.87 \times 10^{7} \mathrm{~s}(\sim 332$ days $)$ when flow rate began to increase

- DBT32A - no fit obtained; increasing flow rate

- DBT32B - no fit obtained; increasing flow rate

- DBT32C - fit only to period of declining flux from 203 to 322 days (referenced to time of enlargement at 254 days from initial drilling for DBT32A); deleted 27 zero-flux values from time of enlargement to time of first measured brine accumulation; deleted all data for $t>2.76 \times 10^{7} \mathrm{~s}(\sim 319$ days) when flow rate began to increase

- L4B01 - no data deleted

- L4X01 - no fit obtained; data show steep, nearly linear decline to zero flux; could not be fit by model that predicts continuously decreasing flow rate

- QPB01 - deleted three outliers: $\left(1.26 \times 10^{6}, 1.02 \times 10^{-11}\right),\left(1.82 \times 10^{6}, 7.41 \times 10^{-11}\right)$, and $\left(1.43 \times 10^{7}, 2.38 \times 10^{-10}\right)$; deleted all data for $t>3.32 \times 10^{7} \mathrm{~s}(\sim 384$ days) when flow rate began to increase

- QPB02 - deleted all data for $t>4.01 \times 10^{6} \mathrm{~s}$ ( 46 days) when flow rate began to decrease rapidly, reached zero, and subsequently increased

- QPB03 - deleted three initial zeroes: $\left(7.72 \times 10^{4}, 0\right),\left(1.97 \times 10^{5}, 0\right)$, and $\left(3.77 \times 10^{5}\right.$, $0)$; deleted one additional early-time zero: $\left(1.41 \times 10^{6}, 0\right)$; deleted all data for $t>1.91 \times$ $10^{7} \mathrm{~s}(\sim 221$ days) when flow rate began to increase

- QPB04 - no fit obtained; no discernible period of continuously decreasing flux

- QPB05 - no fit obtained; no discernible period of continuously decreasing flux 


\subsubsection{Results}

Results of the fits to the full flux histories are summarized in Table 6-1 and shown in Figures 6-1 through 6-15. As observed in Section 2.1, the permeabilities resulting from the fits fall in a reasonably narrow range, typically of order $10^{-21} \mathrm{~m}^{2}$. The diffusivities, however, are widely distributed, with most in the range $10^{-10}$ to $10^{-8} \mathrm{~m}^{2} / \mathrm{s}$, reflecting variability in the time evolution of the seepage from hole to hole. The uncertainties, too, are typically greater for the diffusivities than for the permeabilities, again reflecting significant departures of the flux histories from the model behavior.

The parameter-estimation code ESTIM returns error estimates for the permeability and the diffusivity in the form $k \pm \lambda_{k}$ and $c \pm \lambda_{c}$, respectively, as noted in Section 6.1. The error estimates for the capacitance, $\lambda_{c}$, recorded in Table 5 are computed from:

$$
\lambda_{2}^{2}=C^{2}\left[\left(\frac{\lambda_{k}}{k}\right)^{2}+\left(\frac{\lambda_{c}}{c}\right)^{2}\right]
$$

Note that the analysis reported here treats the brine density, brine viscosity, and borehole radius and length as known constants; (i.e., the error estimates do not include the contributions due to uncertainty in these parameters). However, such contributions are small compared to the uncertainty in the flux and volume measurements, and the ability of the idealized model to match the data.

An additional measure of the average properties of the salt, as well as a measure of uncertainty for several holes as a group, can be obtained from a fit that lumps the data for a number of holes. This has been done for all the 4-inch-diameter $(a=0.051 \mathrm{~m})$ holes in Room D, with the exception of DBT14, which exhibited an increasing flux over most of the observation period. Data for DBT10, DBT11, DBT12, DBT13, DBT15A, DBT15B, DBT31A, and DBT32A were included, and eight zero-flux values at early time were removed, in accord with the discussion in the foregoing section. The fit was then performed on the remaining 582 points. The results are:

$$
\begin{aligned}
& k=(0.57 \pm 0.021) \times 10^{-21} \mathrm{~m}^{2}, \\
& c=(9.82 \pm 1.04) \times 10^{-10} \mathrm{~m}^{2} / \mathrm{s}, \\
& C=(0.36 \pm 0.040) \times 10^{-9} \mathrm{~Pa}^{-1},
\end{aligned}
$$

The fit is compared to the data in Figure 6-16. 
Table 6-1. Parameters Derived from Fits to Full Flux.

\begin{tabular}{|c|c|c|c|c|}
\hline Hole & $\begin{array}{c}\text { Perm. } \times \text { Press. } \\
k_{p_{\infty}} \\
\left(\mathrm{m}^{2} \mathrm{~Pa}, \times 10^{-15}\right)\end{array}$ & $\begin{array}{c}\text { Permeability* } \\
k \\
\left(\mathrm{~m}^{2}, \times 10^{-21}\right)\end{array}$ & $\begin{array}{c}\text { Diffusivity } \\
c \\
\left(\mathrm{~m}^{2} / \mathrm{s}, \times 10^{-10}\right)\end{array}$ & $\begin{array}{c}\text { Capacitance* } \\
C \\
\left(\mathrm{~Pa}^{-1} \times 10^{-9}\right)\end{array}$ \\
\hline DBT10 & $3.84 \pm 0.24$ & $0.38 \pm 0.024$ & $0.47 \pm 0.078$ & $3.87 \pm 0.69$ \\
\hline DBT11 & $14.92 \pm 0.55$ & $1.49 \pm 0.055$ & $35.09 \pm 6.29$ & $0.20 \pm 0.037$ \\
\hline DBT12 & $8.40 \pm 0.94$ & $0.84 \pm 0.094$ & $101.73 \pm 65.33$ & $0.039 \pm 0.025$ \\
\hline DBT13 & $2.29 \pm 0.35$ & $0.23 \pm 0.035$ & $0.59 \pm 0.23$ & $1.85 \pm 0.77$ \\
\hline DBT14A & $10.25 \pm 3.13$ & $1.02 \pm 0.31$ & $278.10 \pm 456.79$ & $0.018 \pm 0.031$ \\
\hline DBT14B & $29.26 \pm 3.67$ & $2.93 \pm 0.37$ & $433.61 \pm 329.03$ & $0.032 \pm 0.025$ \\
\hline DBT15A & $4.23 \pm 0.72$ & $0.42 \pm 0.072$ & $1.85 \pm 0.86$ & $1.09 \pm 0.54$ \\
\hline DBT15B & $2.38 \pm 0.77$ & $0.24 \pm 0.077$ & $1.27 \pm 1.22$ & $0.89 \pm 0.90$ \\
\hline DBT31A & $11.76 \pm 3.10$ & $1.18 \pm 0.31$ & $4.05 \pm 3.36$ & $1.38 \pm 1.20$ \\
\hline DBT31C & $0.037 \pm 1.34$ & $0.0037 \pm 0.134$ & $0.0034 \pm 0.245$ & $5.24 \pm 422.59$ \\
\hline DBT32C & $0.12 \pm 3.18$ & $0.012 \pm 0.318$ & $0.0045 \pm 0.233$ & $13.07 \pm 760.22$ \\
\hline LAB01 & $0.88 \pm 0.56$ & $0.088 \pm 0.056$ & $0.58 \pm 0.91$ & $0.73 \pm 1.24$ \\
\hline L4X01 & - & - & - & - \\
\hline QPB01 & $18.76 \pm 1.18$ & $1.88 \pm 0.118$ & $110.04 \pm 34.07$ & $0.081 \pm 0.026$ \\
\hline QPB02 & $321.34 \pm 1.25$ & $32.13 \pm 0.12$ & $11.58 \pm 0.14$ & $13.22 \pm 0.17$ \\
\hline QPB03 & $19.06 \pm 5.93$ & $1.91 \pm 0.59$ & $6388 \pm 18839$ & $0.0014 \pm 0.0042$ \\
\hline QPB04 & - & - & - & - \\
\hline QPB05 & - & - & - & - \\
\hline
\end{tabular}

* Assumes $p_{\infty}=1.0 \times 10^{7} \mathrm{~Pa}, \mu=2.1 \times 10^{-3} \mathrm{~Pa} \cdot \mathrm{s}$.

Holes DBT16 and DBT17 yielded no brine, and are not included in the table. Blank entries indicate failure to obtain a fit.

A similar exercise grouped data from all holes in the Room Q Access Drift, with the exception of QPB02, which produced brine at a rate one to two orders of magnitude greater than nearby holes. The fit combines data from QPB01, QPB03, QPB04, and QPB05. Data for $t>3 \times 10^{7} \mathrm{~s}$, as well as 30 zero-flux values at early time, were deleted, leaving 112 points. The results are:

$$
\begin{aligned}
& k=(0.51 \pm 0.034) \times 10^{-21} \mathrm{~m}^{2}, \\
& c=(6.44 \pm 1.48) \times 10^{-10} \mathrm{~m}^{2} / \mathrm{s}, \\
& C=(0.37 \pm 0.090) \times 10^{-9} \mathrm{~Pa}^{-1},
\end{aligned}
$$




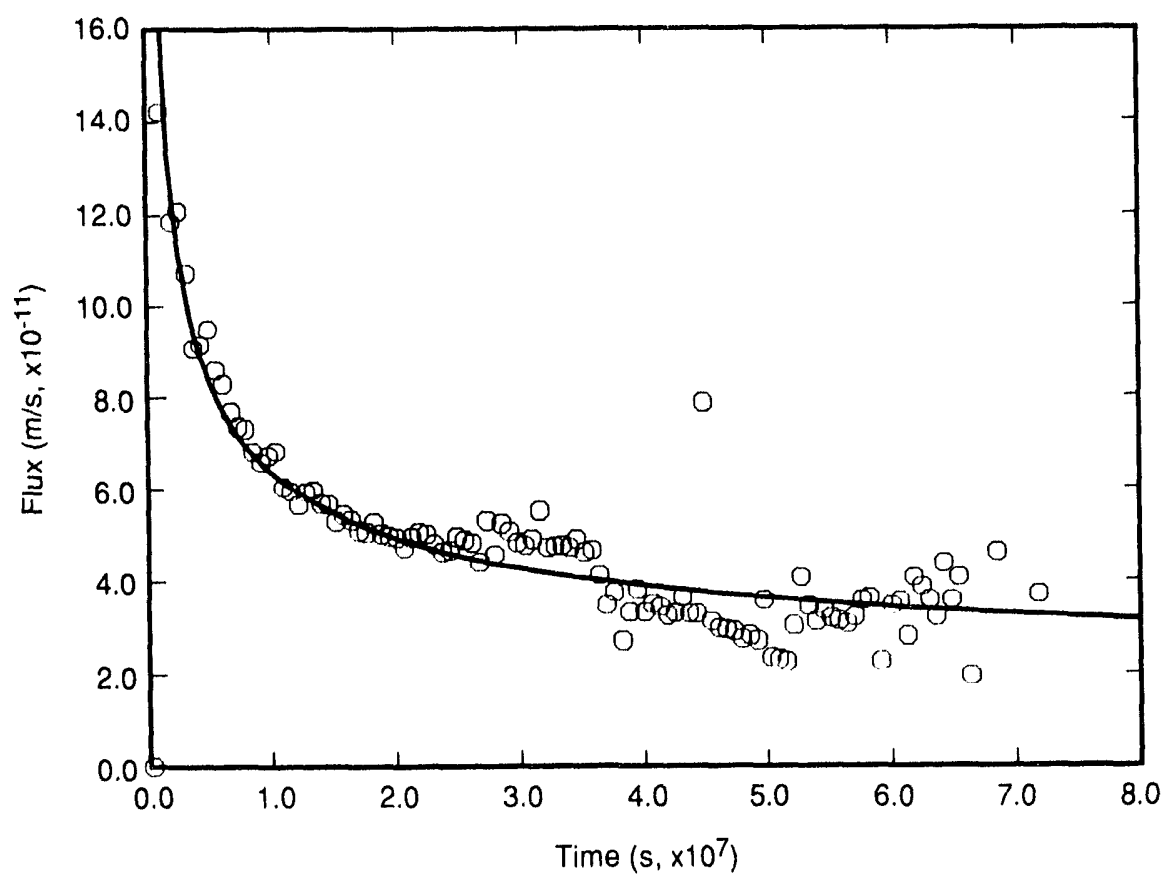

TRI-6119-281-0

Figure 6-1. Comparison of model fit to data for full flux history, hole DBT10.

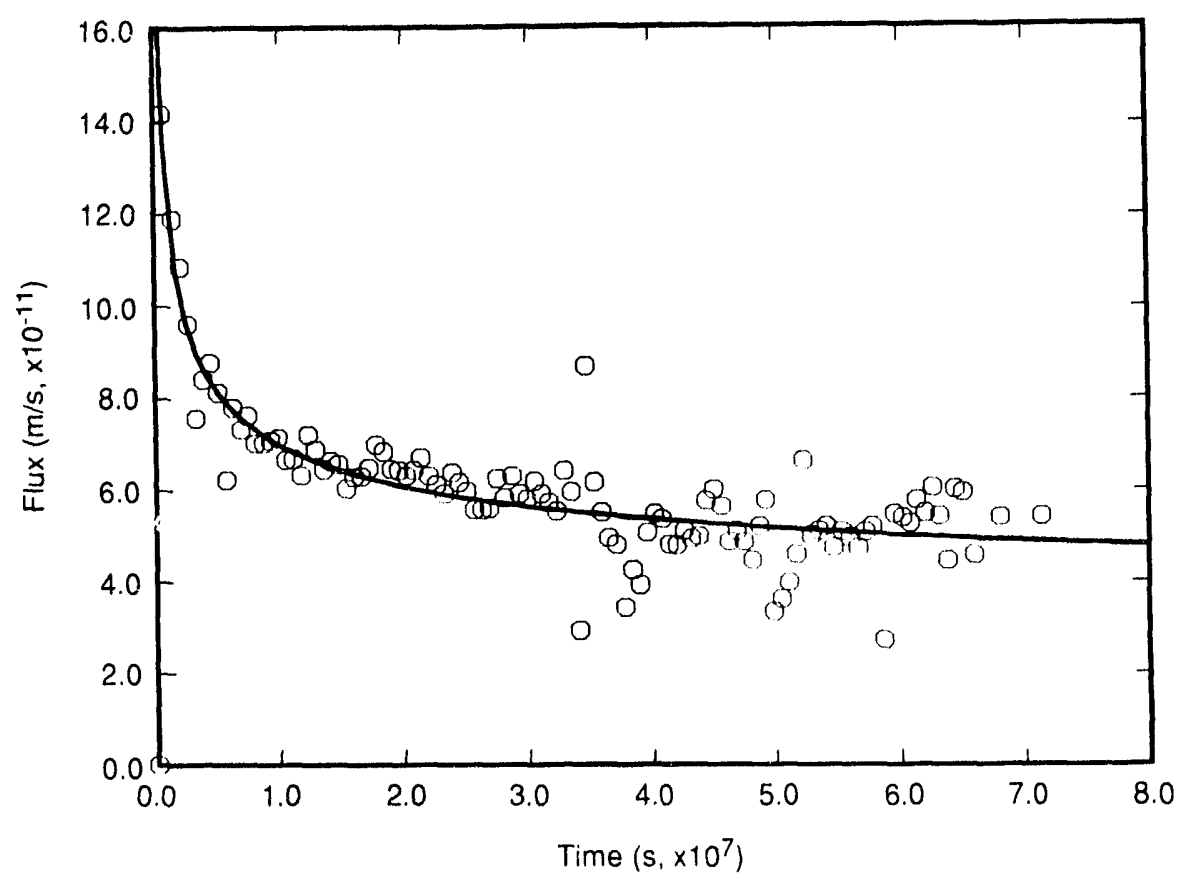

TRI-6119-282-0

Figure 6-2. Comparison of model fit to data for full flux history, hole DBT11. 


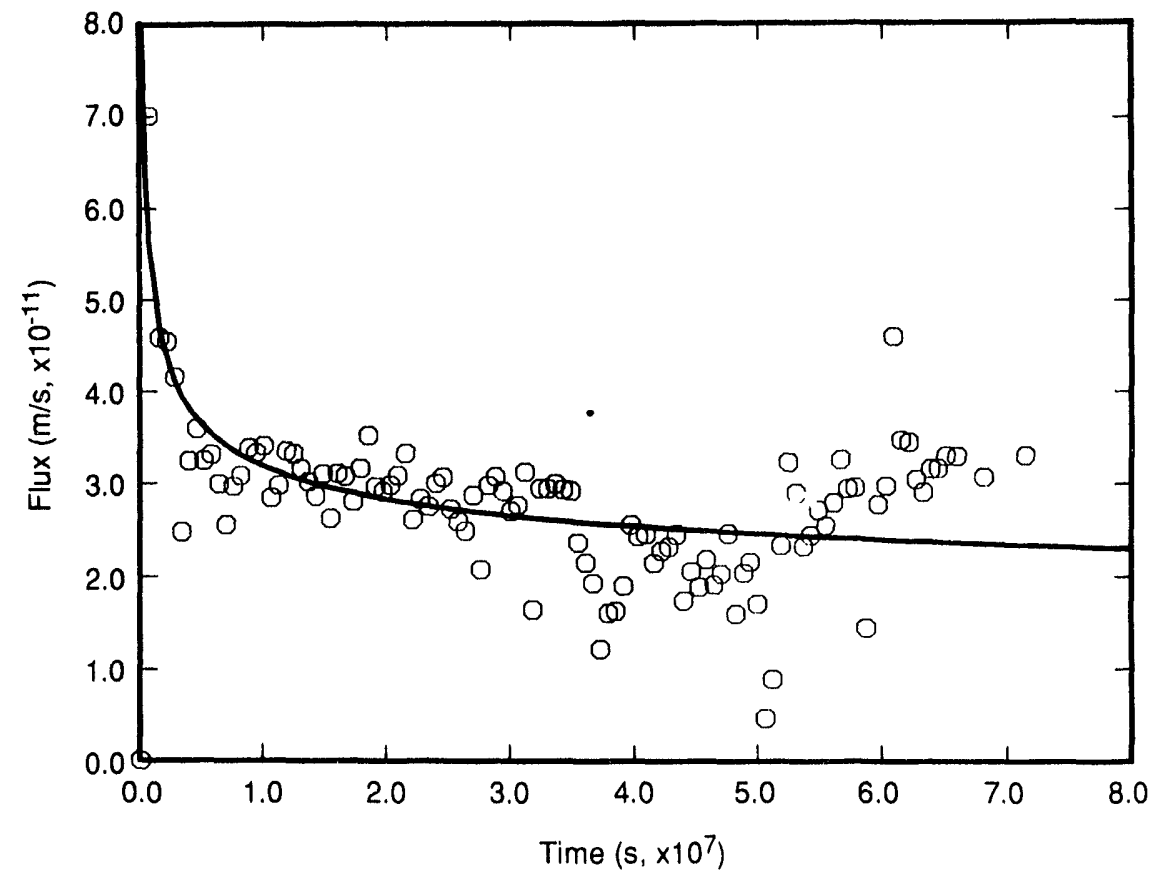

TRI-6119-283.0

Figure 6-3. Comparison of model fit to data for full flux history, hole DBT12.

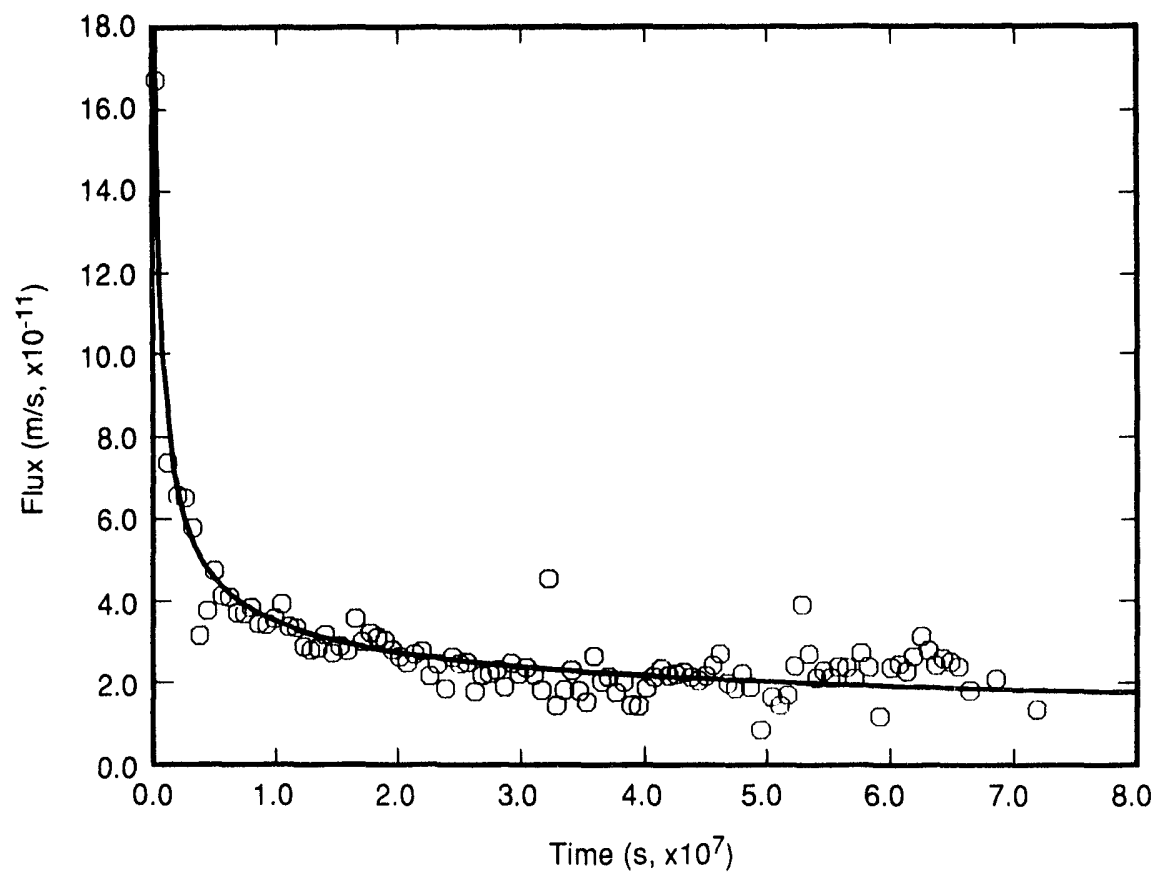

Figure 6-4. Comparison of model fit to data for full flux history, hole DBT13. 


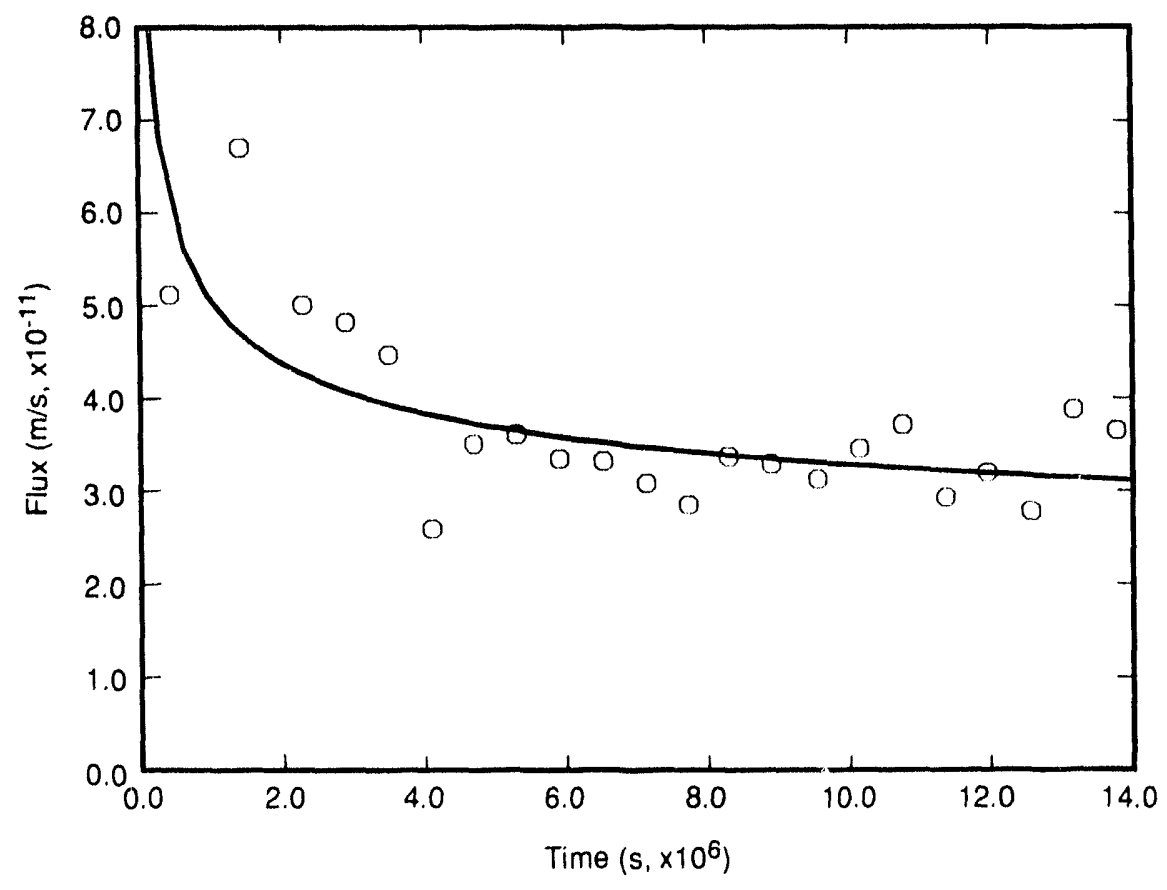

TRI.6119-285-0

Figure 6-5. Comparison of model fit to data for full flux history, hole DBT14A.

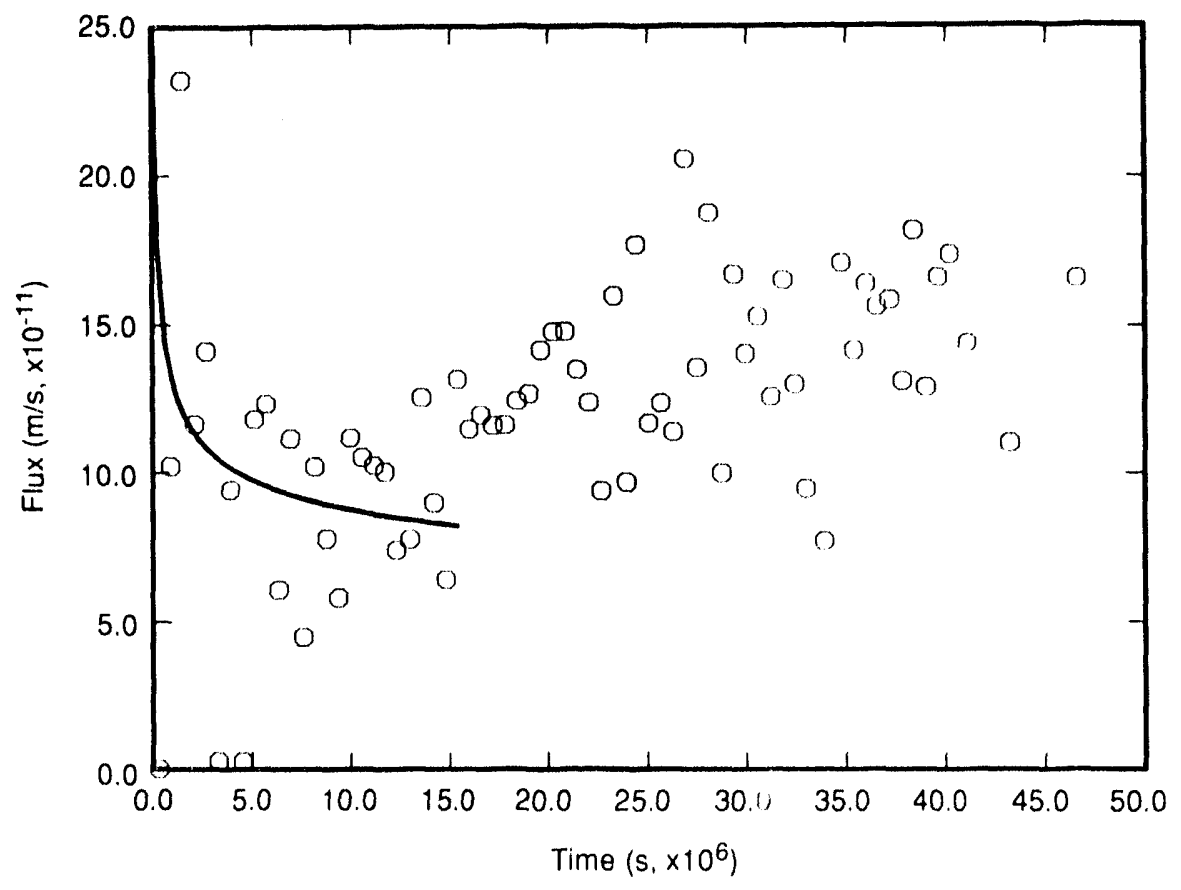

Figure 6-6. Comparison of model fit to data for full flux history, hole DBT14B. 


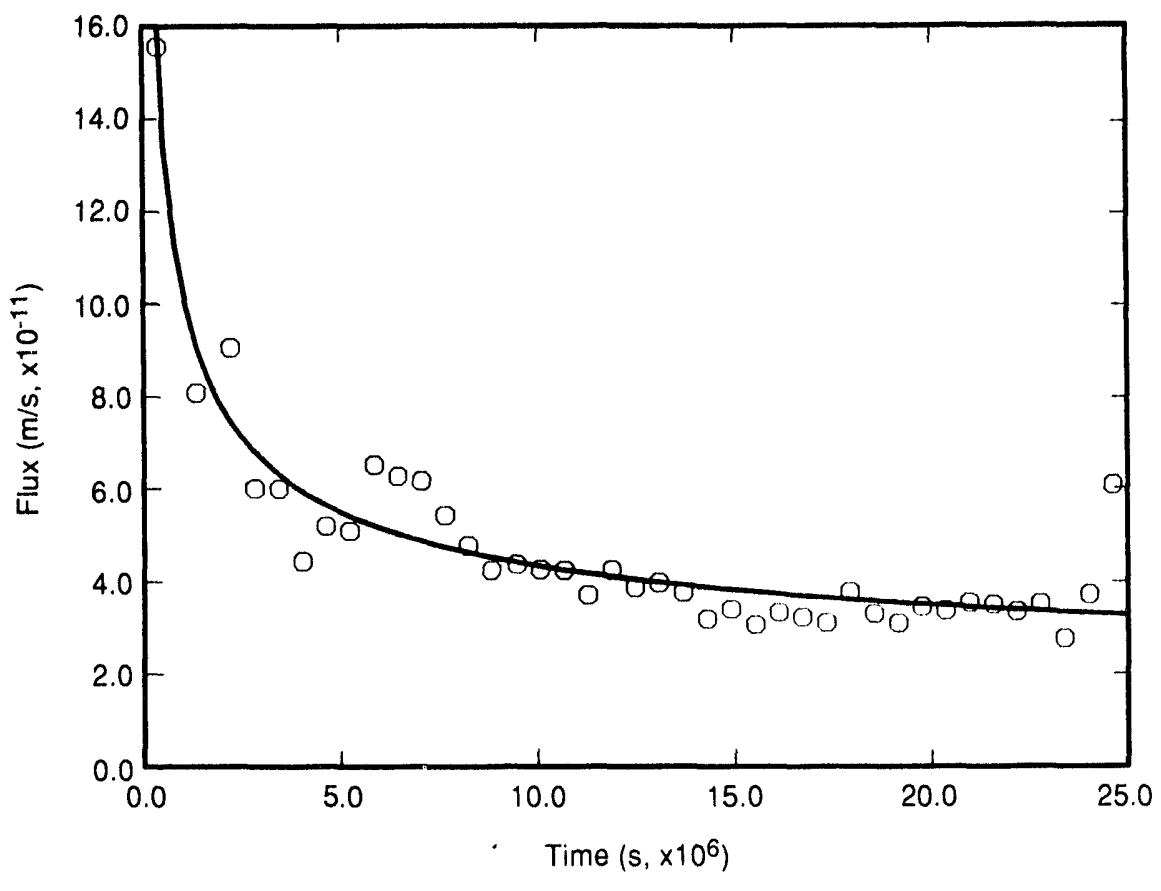

TRI-6119.287-0

Figure 6-7. Comparison of model fit to data for full flux history, hole DBT15A.

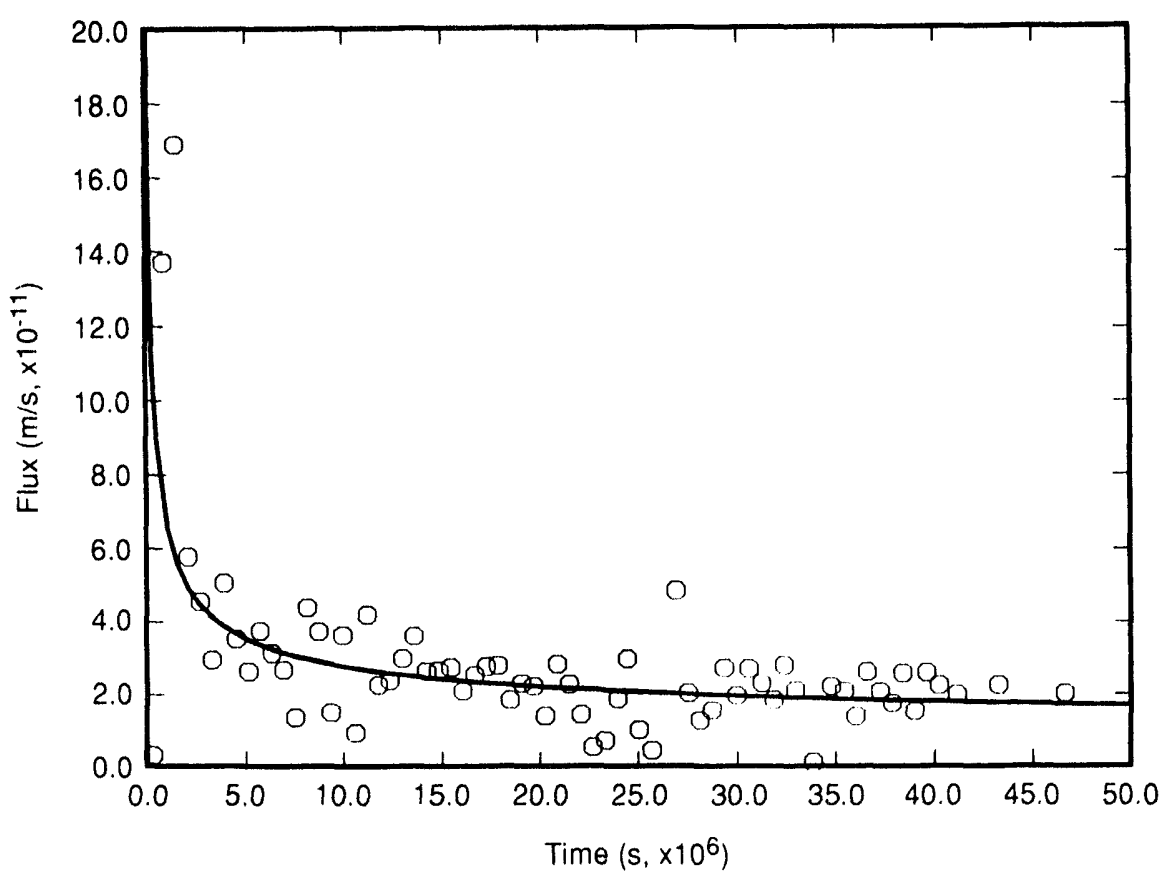

Figure 6-8. Comparison of model fit to data for full flux history, hole DBT15B. 


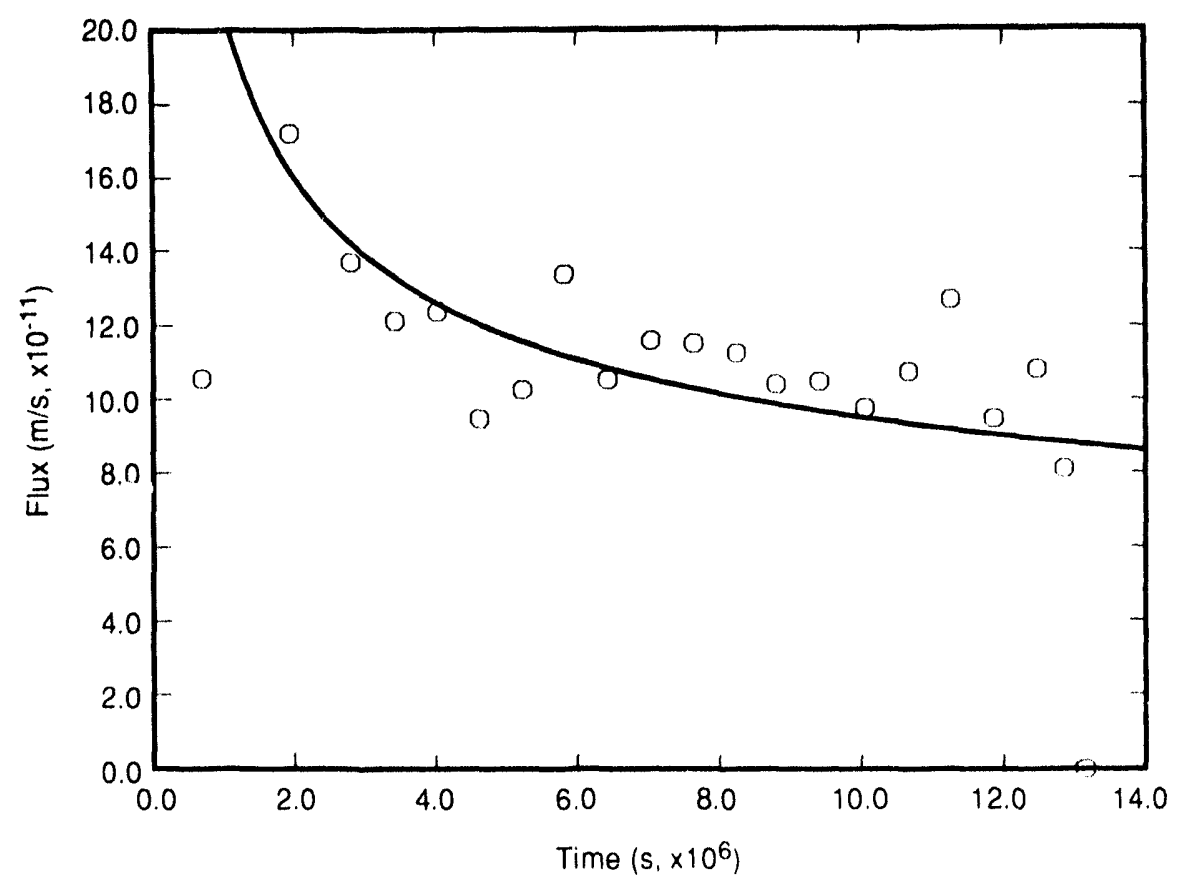

TRI.6119-289.0

Figure 6-9. Comparison of model fit to data for full flux history, hole DBT31A.

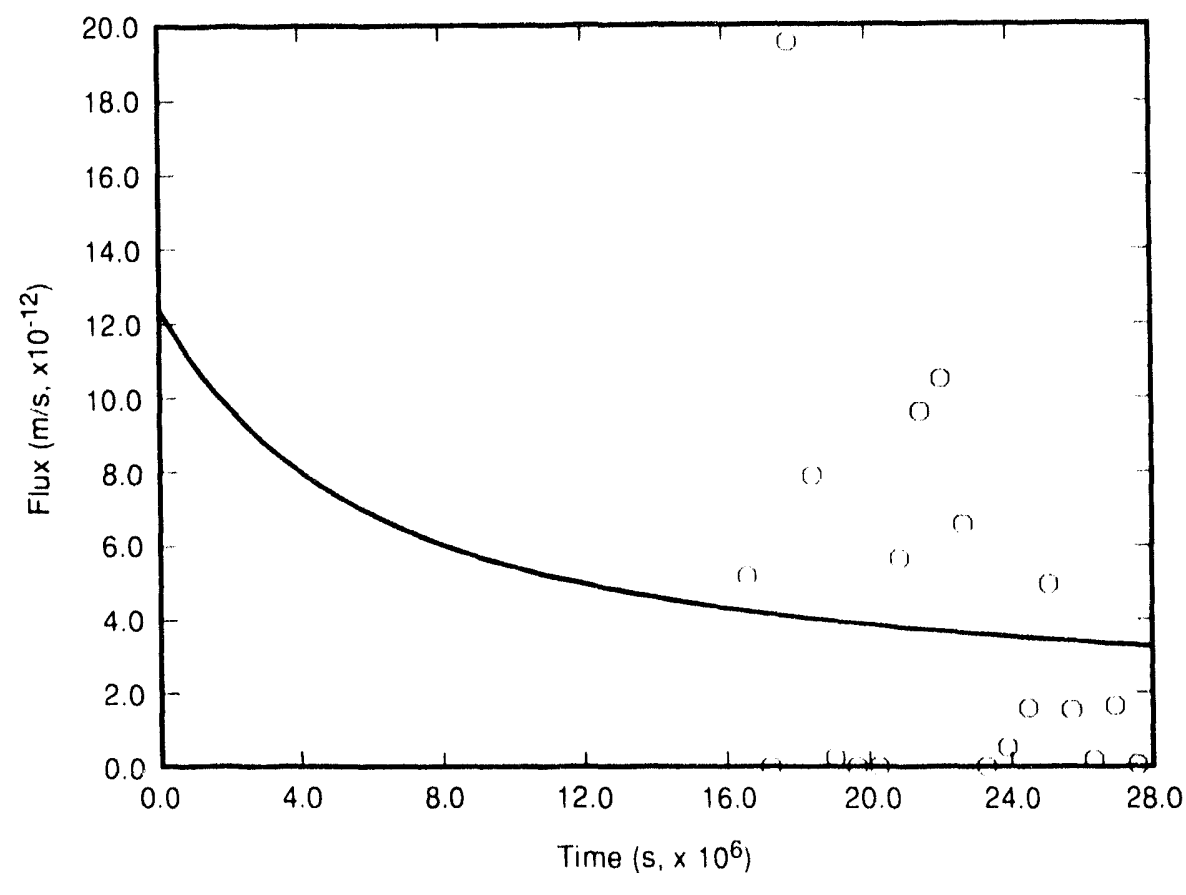

TRI.6119.290.0

Figure 6-10. Comparison of model fit to data for full llux history, hole DBT3IC. 


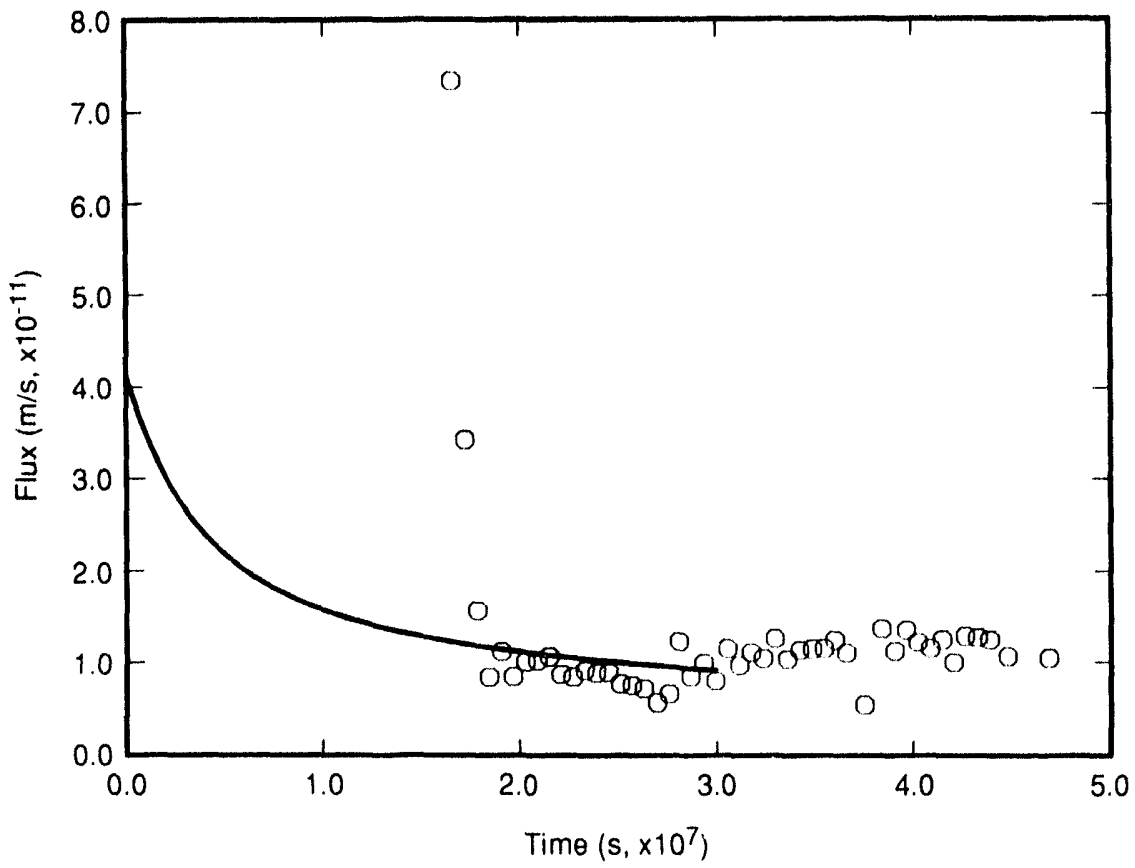

TRI.6119-291.0

Figure 6-11. Comparison of model fit to data for full flux history, hole DBT32C.

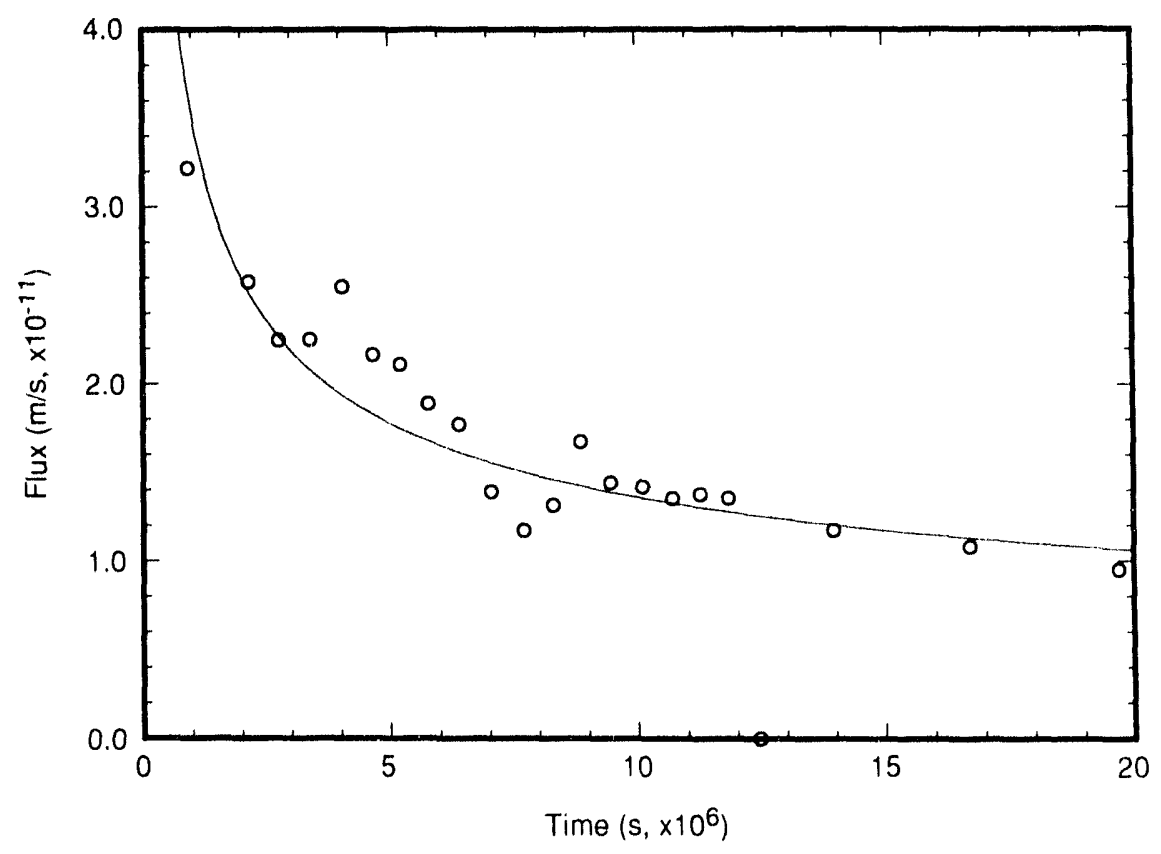

TRI-61192920

Figure 6-12. Comparison of model fit to data for full flux history, hole L4B01. 


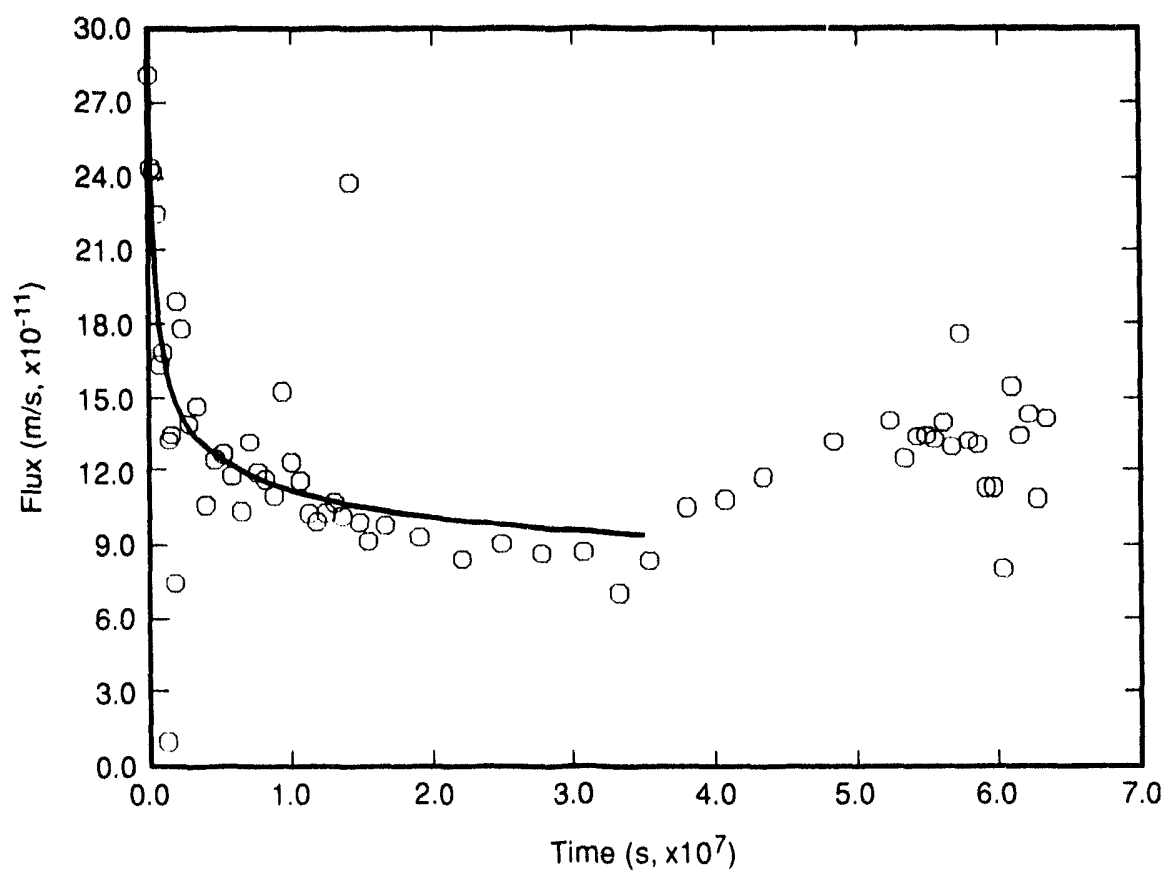

Figure 6-13. Comparison of model fit to data for full flux history, hole QPB01.

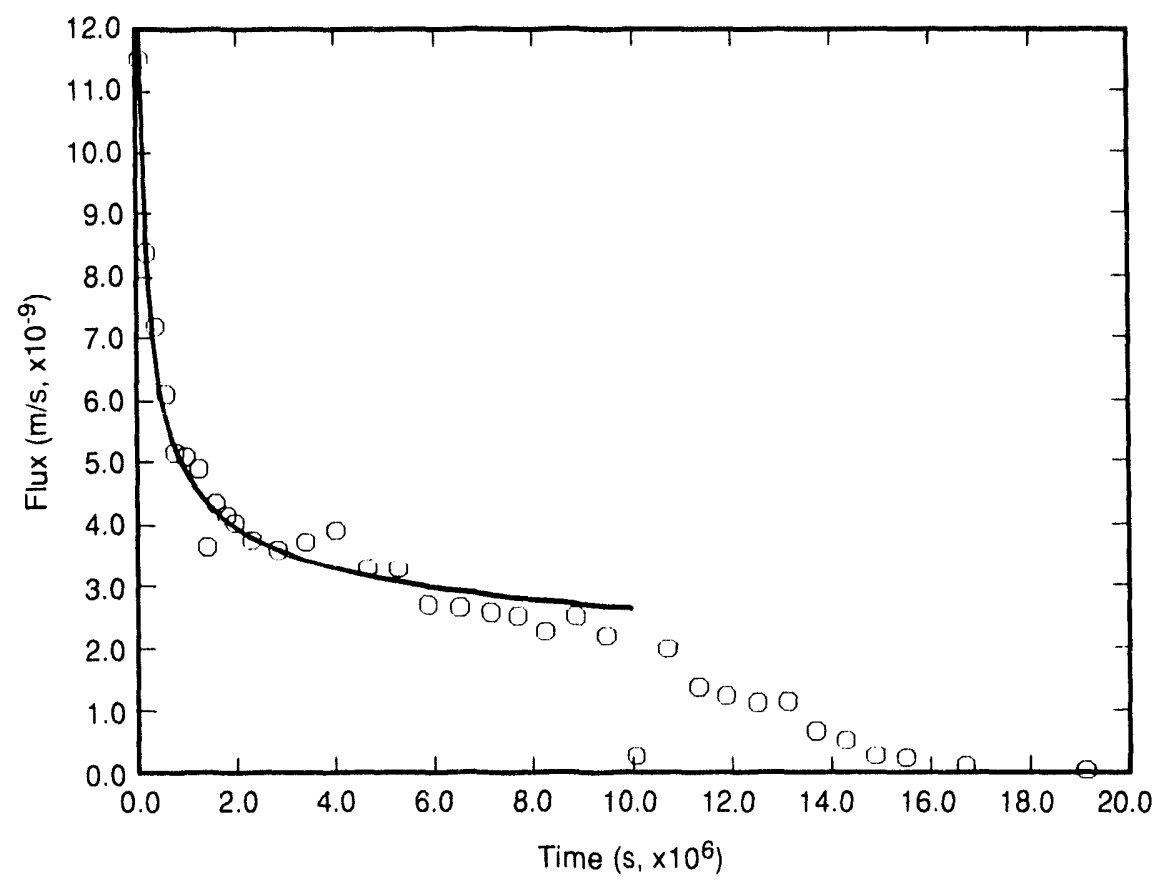

Figure 6-14. Comparison of model fit to data for full flux history, hole QPB02. 


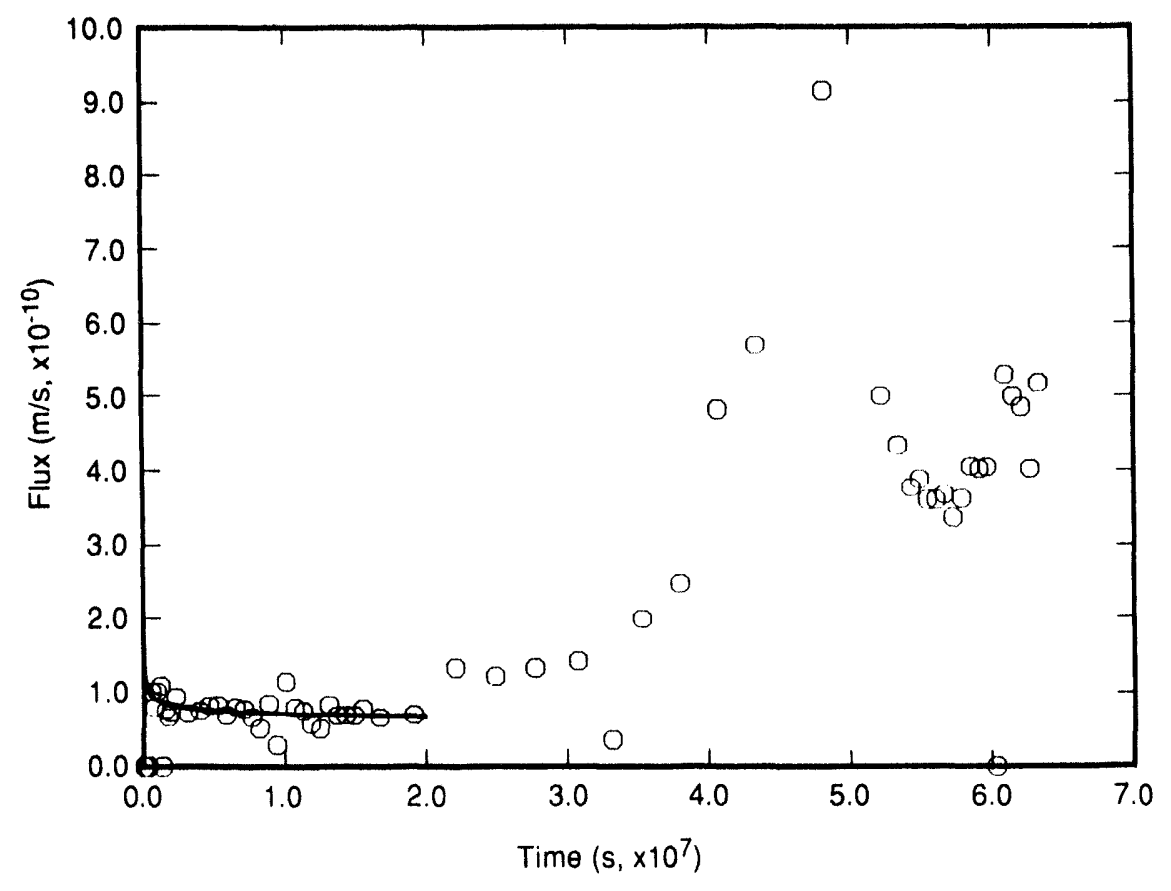

TRI-6119.295.0

Figure 6-15. Comparison of model fit to data for full flux history, hole QPB03.

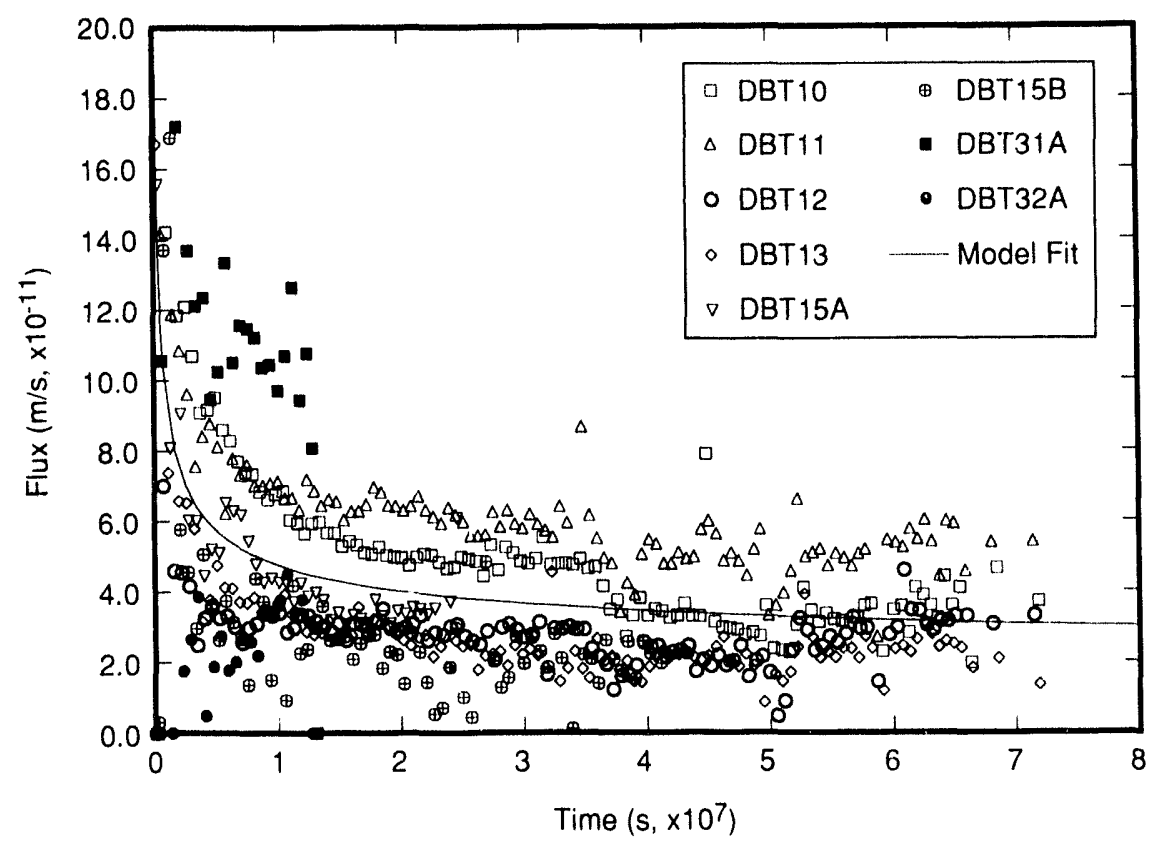

TRI.6119-296-0

Figure 6-16. Comparison of model fit to data for full flux history, Room D holes grouped. 
The fit is compared to the data in Figure 6-17.

\subsection{Fits Based on the Full Cumulative Volume Histories}

This section describes fits based on the cumulative volume history for each hole (Equation 36). This exercise offers two advantages over fitting data for the flux. First, the raw data are in the form of the volume collected in each sampling. Thus, data for cumulative volume versus time can be constructed directly. In contrast, assembly of data for the flow rate involves some sort of approximation (see Section 6.2). Second, the data for cumulative volume are much smoother than those for the flow rate. The cumulative volume is, by definition, a non-decreasing function, while approximations to its derivative with respect to time can be highly variable.

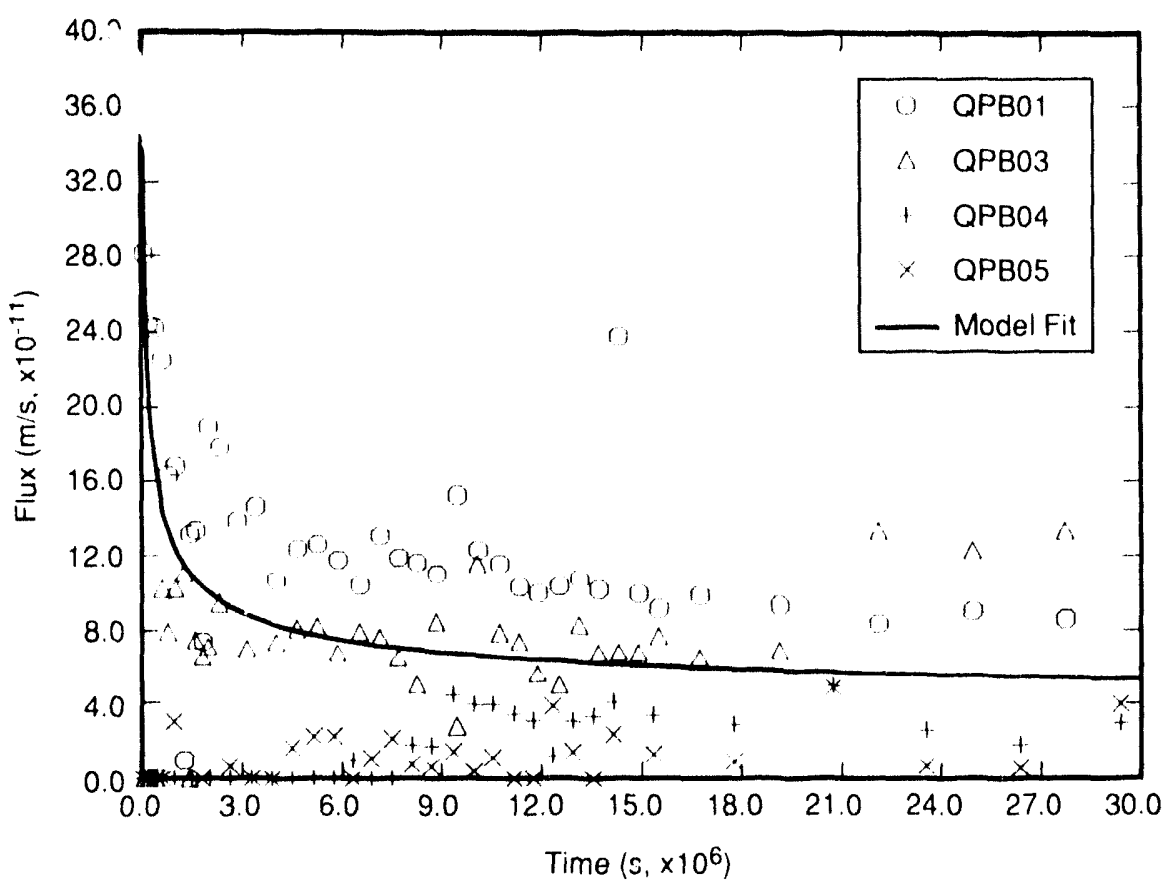

Figure 6-17. Comparison of model fit to data for full flux history, Room Q Access Drift holes grouped. 
Fits to the flux and to the cumulative volume for the same borehole will yield somewhat different parameter values, in part due to the different weights given to different portions of the data in the two schemes. In particular, a fit to the cumulative volume tends to weight the late-time data (when the volume is large), while a fit to the flux tends to weight the early-time data (when the flux is large).

The fits to the cumulative volume data were again performed with the parameter-estimation code ESTIM (see Section 6.1). A subroutine was written to evaluate Equation (36), again leaving two parameters to be determined. As is the case for the flux (Section 6.2), there is one parameter that characterizes the time when the flow evolves, $t_{o}=a^{2} / c$, and one parameter that characterizes the magnitude of the volume (per unit area), $v_{o}=k p_{\infty} a / \mu c=C p_{\infty} a$. With $a$ and $\mu$ known, one may fit for the diffusivity, $c$, and the product of the capacitance and the initial pressure, $C p_{\infty}$. The integral in Equation (36) was evaluated by Gauss quadrature using the SLATEC subroutine DGAUS8.

Hole DBT 15 was elongated after about 295 days. A single fit of the cumulative volume model to the data for DBT15 was performed by assuming that one set of parameters characterizes the initial hole length, and a second set characterizes the additional length. The initial length is assumed to continue producing brine after the extension, and production from the new section is superposed. Thus, four parameters were determined in a single fit, reflecting the magnitude and the time scale of the flow to each section of the hole.

\subsubsection{Results}

Fits were obtained for the entire monitored histories of holes DBT10, DBT11, DBT12, DBT13, DBT15, L4B01, and QPB01. For reasons discussed in detail below, fits could not be obtained for the full data available for DBT14 and QPB02. In these cases, fits were performed to the data for early time. The remaining holes, DBT31, DBT32, L4X01, QPB03, QPB04, and QPB05, exhibited behavior sufficiently erratic that fits could not be obtained using the full histories.

The fits of Equation (36) to data for cumulative volume are shown in Figures 6-18 through 626. The resulting parameters are summarized in Table 6-2. The fit determines $c$ and $p_{\infty} c$; the capacitance is determined from the latter, assuming $p_{\infty}=10^{7} \mathrm{~Pa}$. The permeability is then determined using $k=\mu C c$, with $\mu=2.1 \times 10^{-3} \mathrm{~Pa} \cdot \mathrm{s}$. The diffusivity characterizes the rate of decay of the flux; consequently, it is sensitive to the evolution of the flow in time, which is quite 


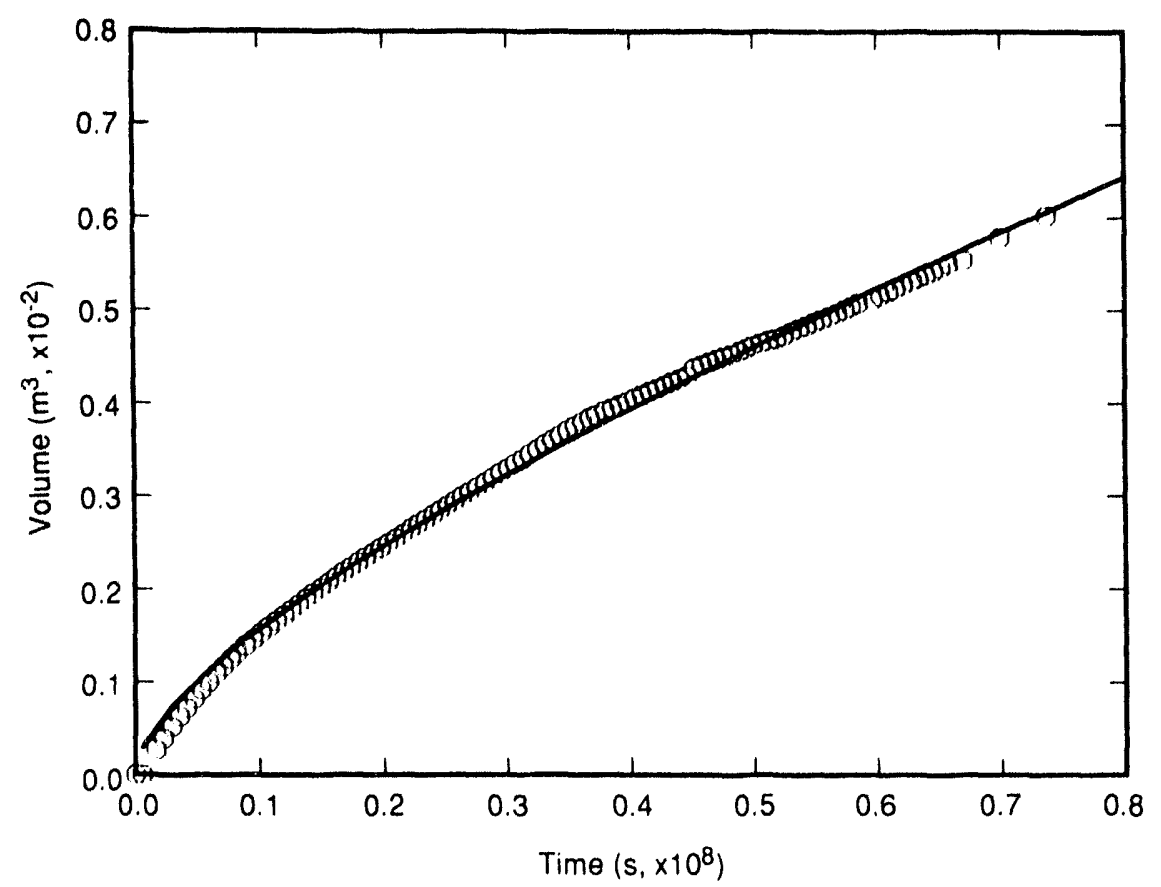

TRI.6119-298.0

Figure 6-18. Comparison of model fit to data for cumulative volume history, hole DBT10.

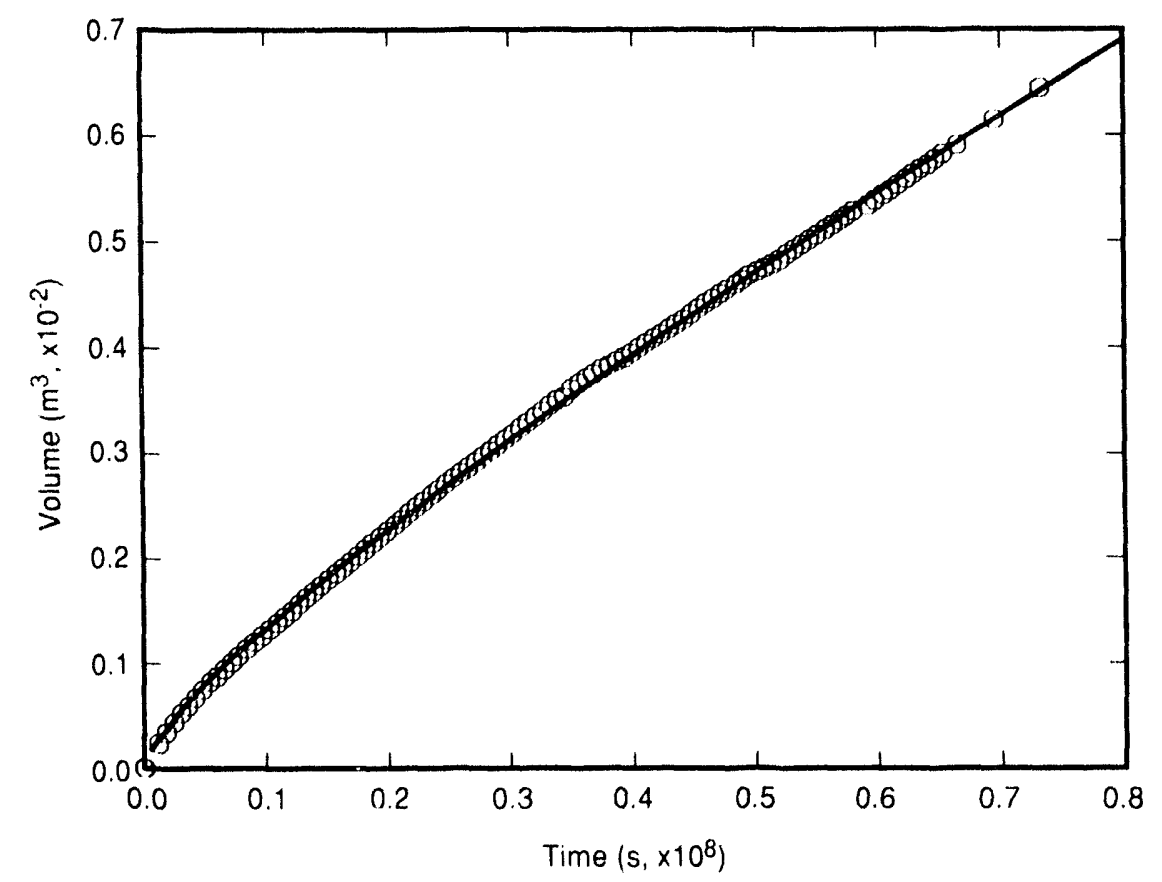

Figure 6-19. Comparison of model fit to data for cumulative volume history, hole DBT11. 


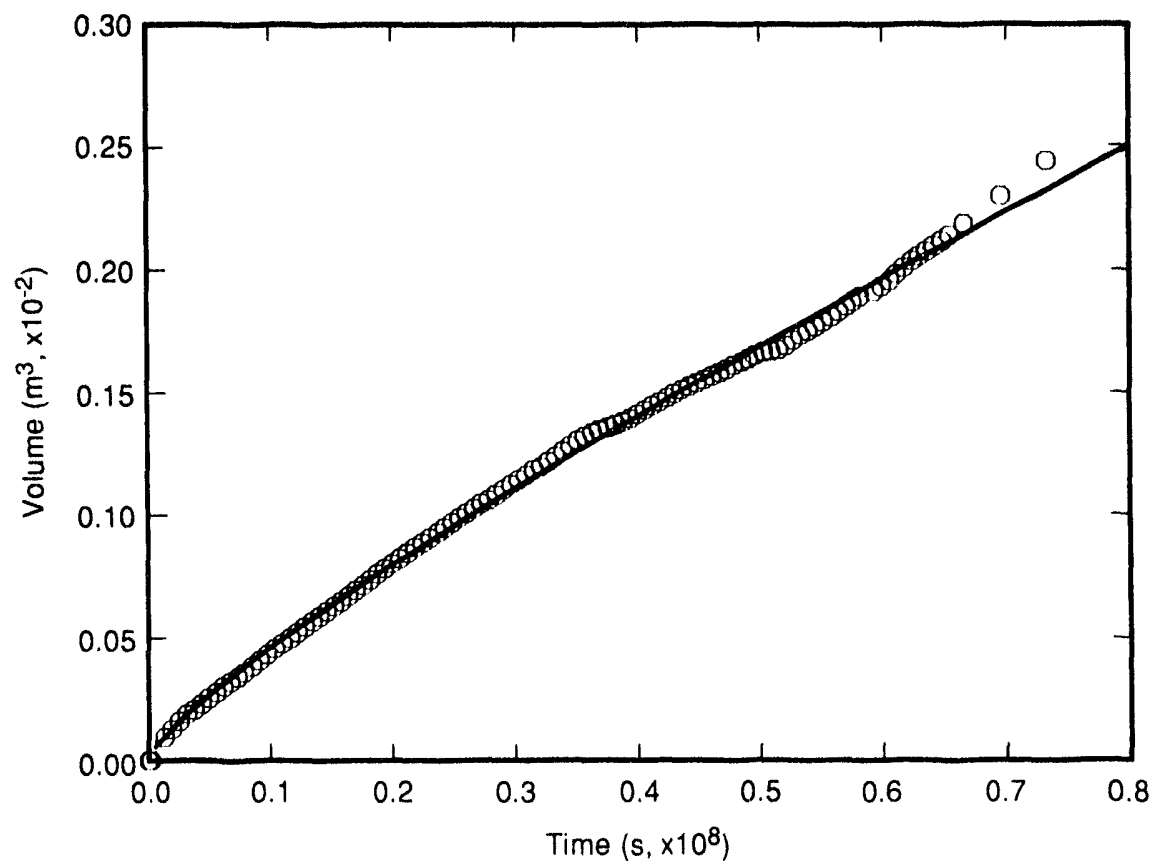

TRI-6119-300-0

Figure 6-20. Comparison of model fit to data for cumulative volume history, hole DBT12.

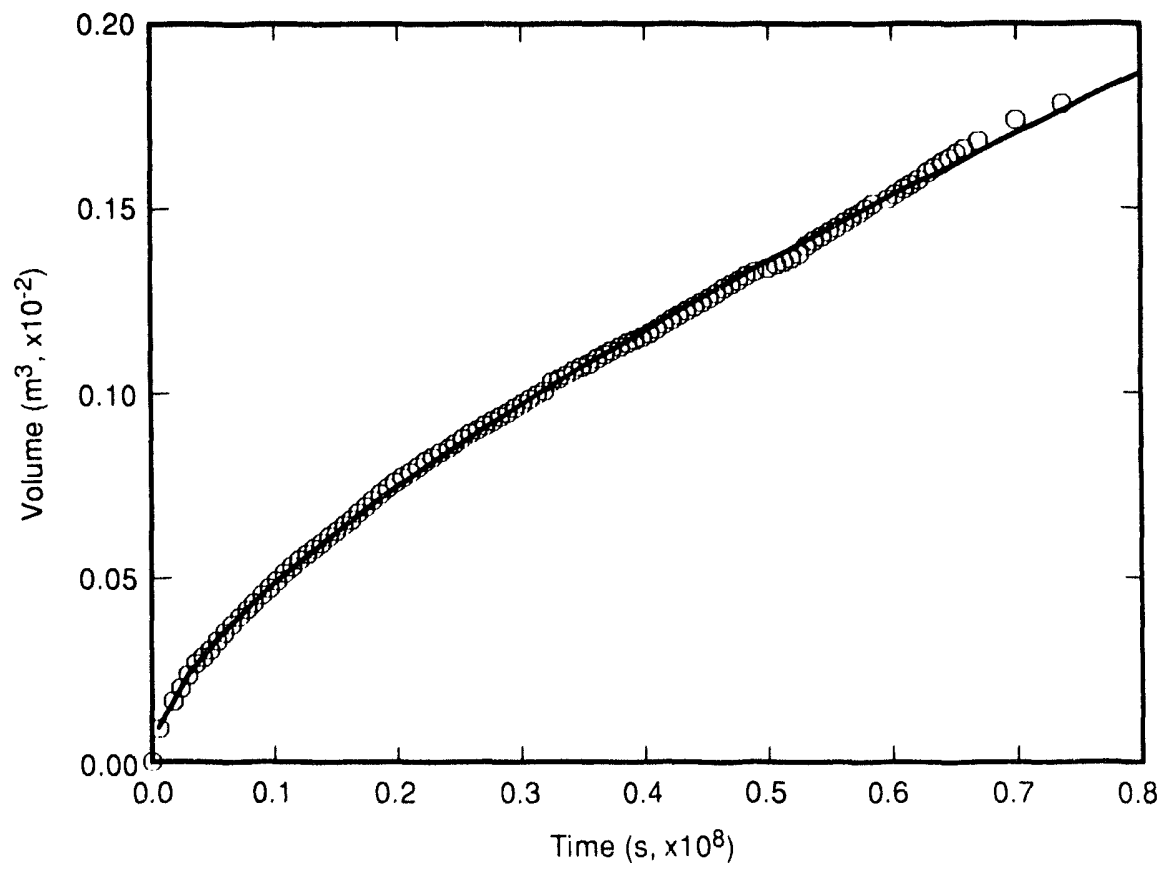

TRI-6119.301-0

Figure 6-21. Comparison of model fit to data for cumulative volume history, hole DBT13. 


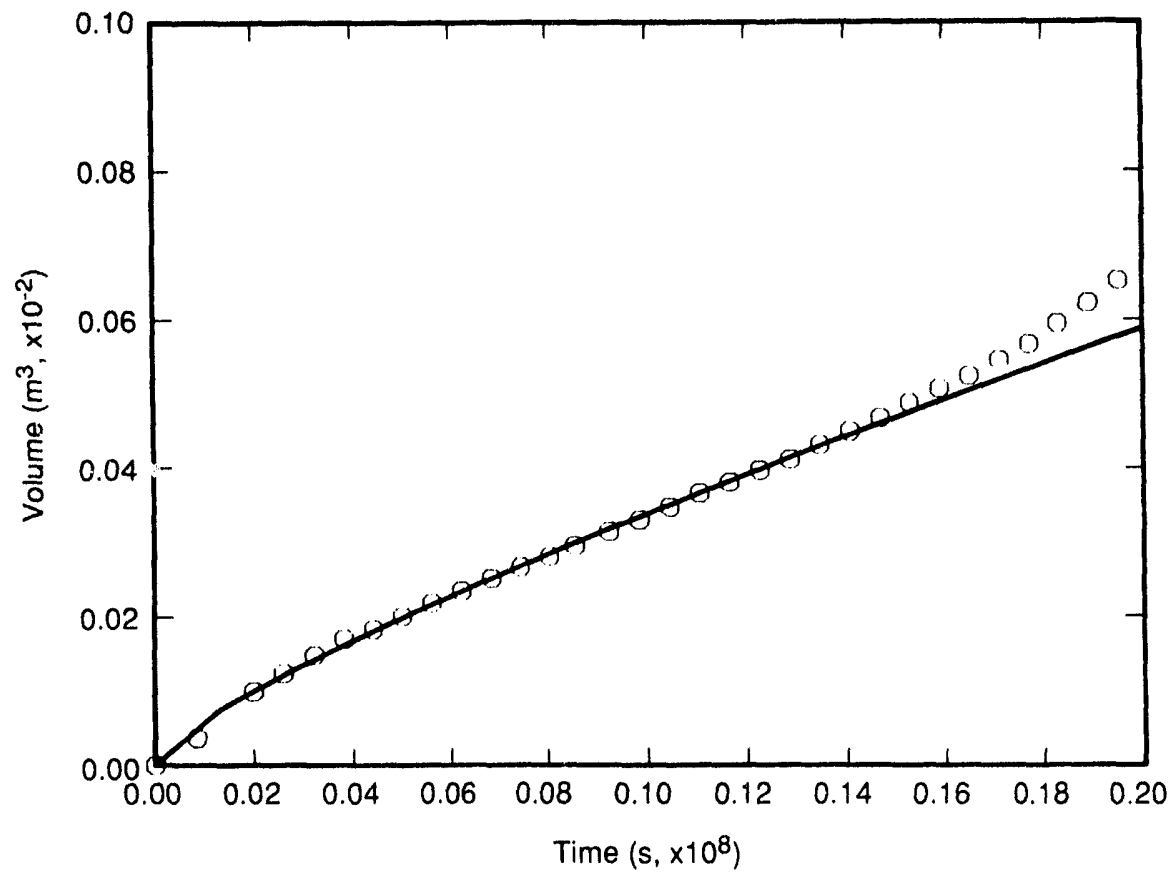

TRI.6119-302-0

Figure 6-22. Comparison of model fit to data for cumulative volume history, hole DBT14A.

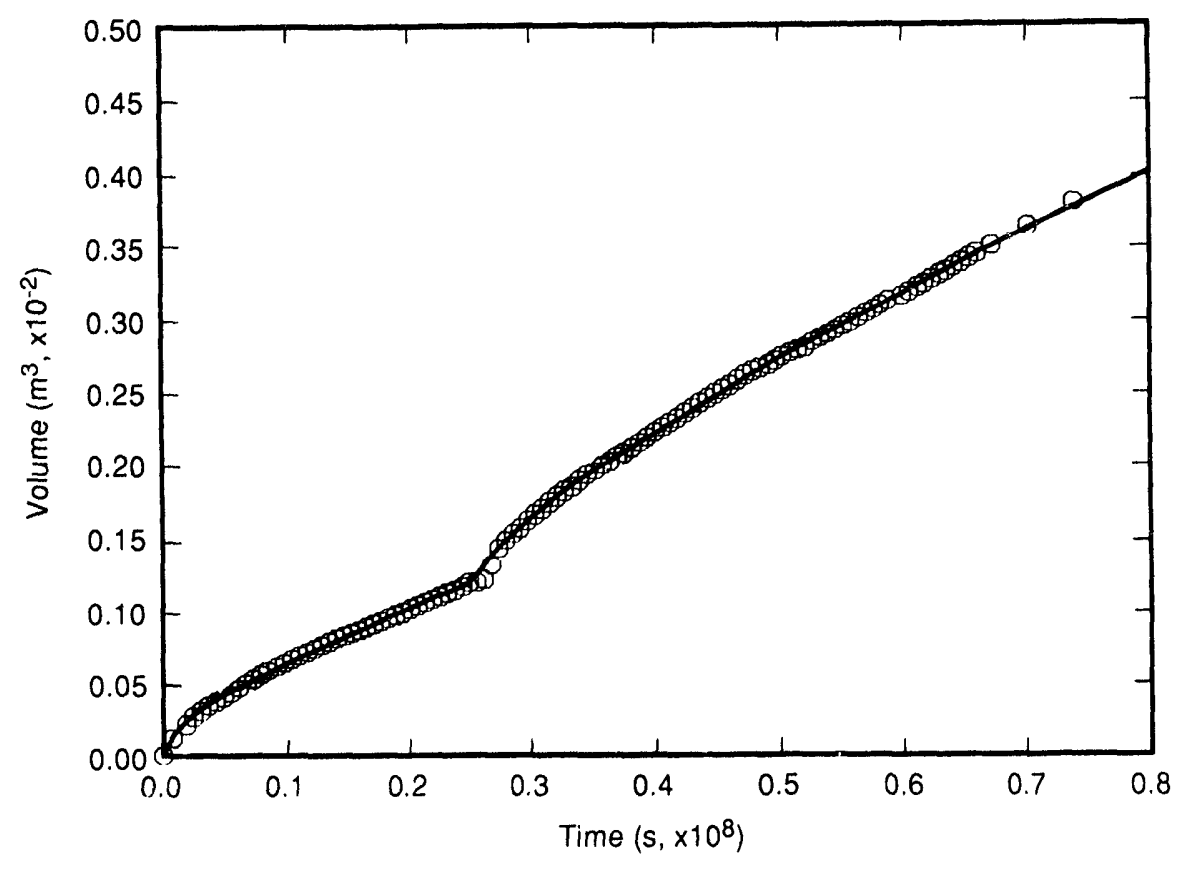

Figure 6-23. Comparison of model fit to data for cumulative volume history, hole DBT15. 


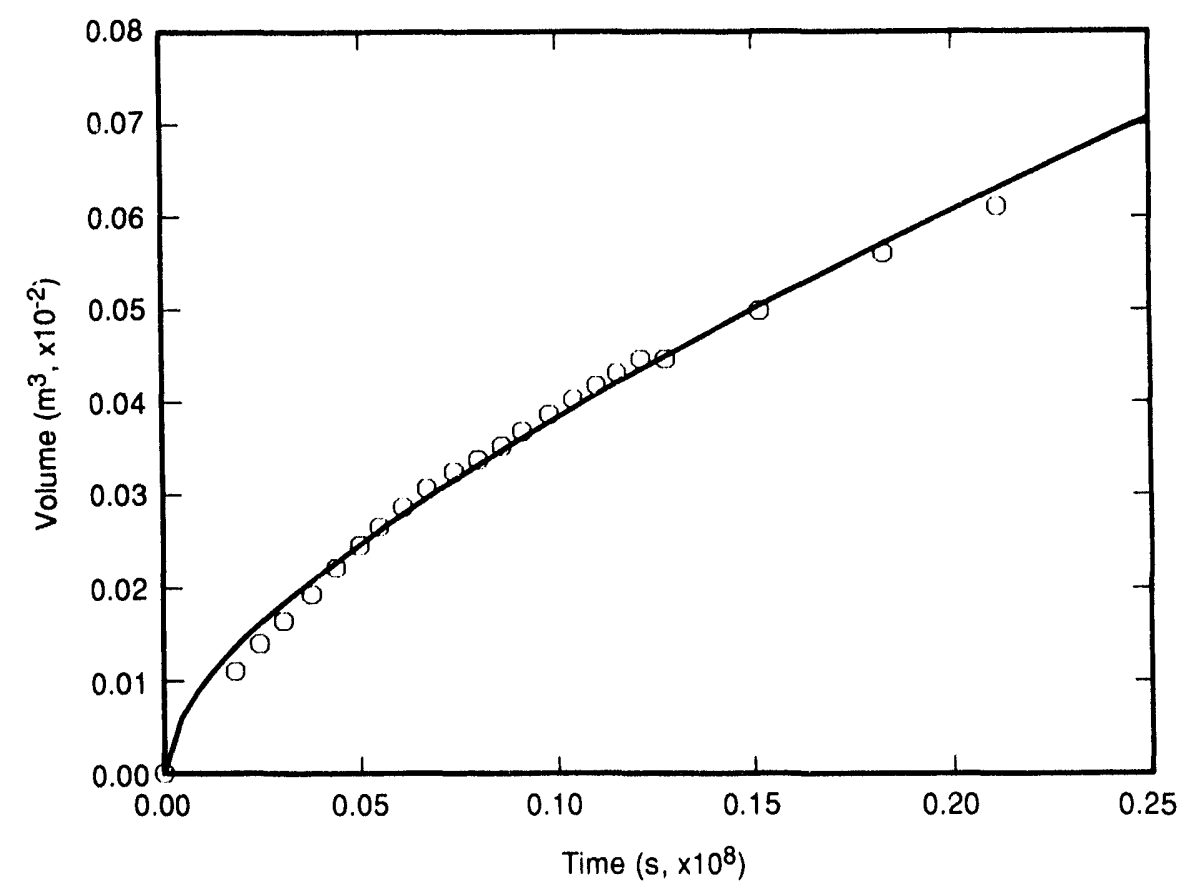

TRI.6119-304-0

Figure 6-24. Comparison of model fit to data for cumulative volume history, hole L4B01.

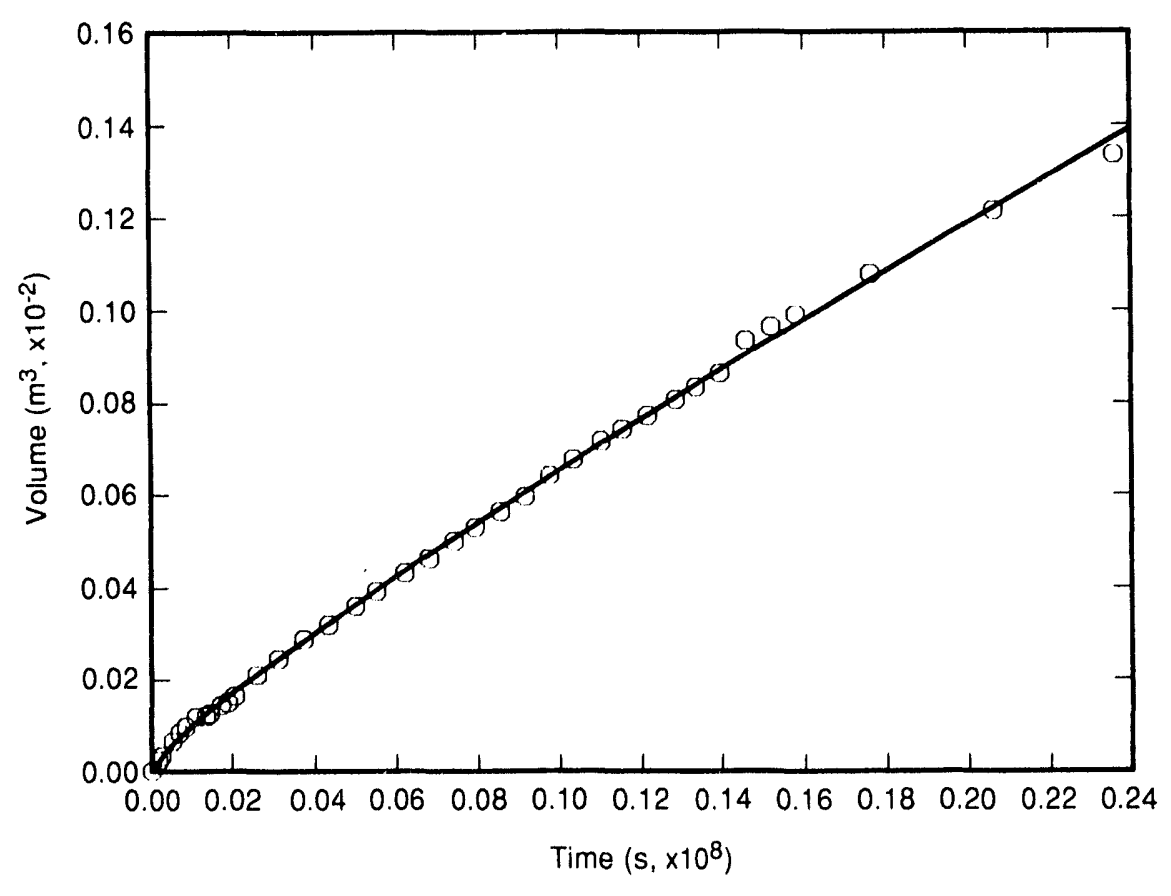

TRI-6119-305-0

Figure 6-25. Comparison of model fit to data for cumulative volume history, hole QPB01. 


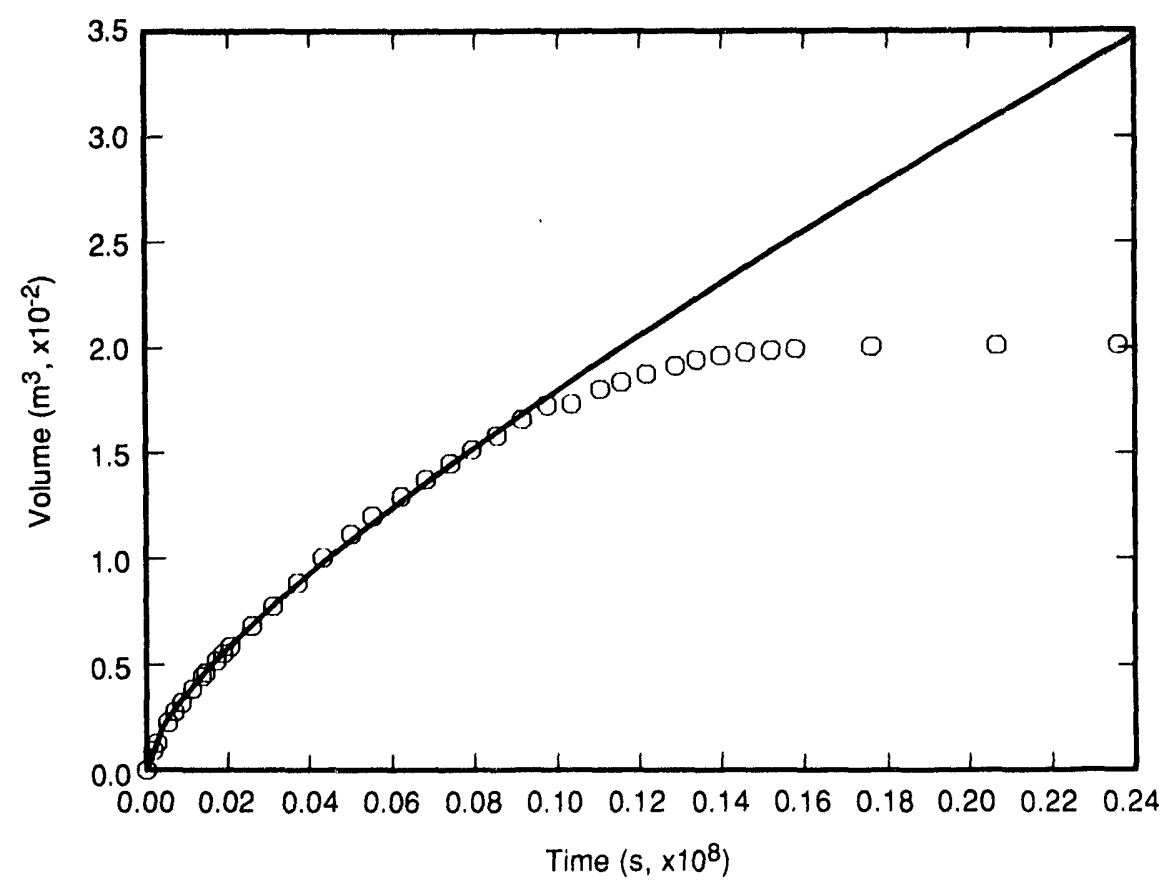

Figure 6-26. Comparison of model fit to data for cumulative volume history, hole QPB02.

variable from hole to hole. Correspondingly, the fits yielded a wide range of apparent diffusivities, of the order of $10^{-10}$ to nearly $10^{-7} \mathrm{~m}^{2} / \mathrm{s}$. Similarly, the inferred capacitance ranges from $10^{-11}$ to $10^{-8} \mathrm{~Pa}^{-1}$. The permeability is determined from the magnitude of the flow rate, which, as noted above, was observed to fall within a fairly narrow range. Thus, the fits yielded permeabilities typically in the range $10^{-22}$ to $10^{-21} \mathrm{~m}^{2}$, with the exception of QPB02, which is of the order of $10^{-20} \mathrm{~m}^{2}$.

\subsection{Fits Based on the Late-Time Flux Histories}

An attempt was also made to fit the late-time, asymptotic solution for the flux (Equation 43) to the data. The disadvantage of this approach is that it discounts the early-time response, when the flow evolves relatively rapidly, and the data contains information more sensitive to the diffusivity. This limitation is manifested in the difficulty of obtaining a reliable value for the intercept $B$, as discussed in Section 5.4. However, there are advantages to considering the late-time response, in addition to its simplicity. First, plots of $|q|^{-1}$ versus $\ln t$ give some visual indication of how far the 
Table 6-2. Parameters Derived from Fits to Cumulative Volume

\begin{tabular}{|c|c|c|c|c|}
\hline Hole & $\begin{array}{c}\text { Diffusivity } \\
c \\
\left(\mathrm{~m}^{2} / \mathrm{s}, \times 10^{-10}\right)\end{array}$ & $\begin{array}{c}\text { Perm. } \times \text { Capac. } \\
p_{\infty} C \\
\left(-, \times 10^{-2}\right)\end{array}$ & $\begin{array}{c}\text { Capacitance* } \\
C \\
\left(\mathrm{~Pa}^{-1}, \times 10^{-9}\right)\end{array}$ & $\begin{array}{c}\text { Permeability* } \\
k \\
\mathrm{~m}^{2},\left(\times 10^{-2 !}\right)\end{array}$ \\
\hline DBT10 & 3.11 & 1.03 & 1.03 & 0.672 \\
\hline DBT11 & 57.7 & 0.133 & 0.133 & 1.61 \\
\hline DBT12 & 141 & 0.0288 & 0.0288 & 0.853 \\
\hline DBT13 & 1.50 & 0.956 & 0.956 & 0.302 \\
\hline $\mathrm{DBT}_{14 \mathrm{~A}^{\dagger}}$ & 133 & 0.0293 & 0.0293 & 0.819 \\
\hline DBT14B & - & - & - & - \\
\hline DBT15A & 4.28 & 0.680 & 0.680 & 0.612 \\
\hline DBT15B & 0.660 & 1.33 & 1.33 & 0.184 \\
\hline DBT31 & - & - & - & 一 \\
\hline DBT32 & - & - & - & - \\
\hline LAB0I & 44.0 & 0.185 & 0.185 & 0.171 \\
\hline L4X01 & - & - & - & - \\
\hline QPB01 & 536 & 0.0212 & 0.0212 & 2.39 \\
\hline QPB02 $\ddagger$ & 9.62 & 14.4 & 14.4 & 29.1 \\
\hline QPB03 & - & - & - & - \\
\hline QPB04 & - & - & - & 一 \\
\hline QPB05 & - & - & - & - \\
\hline
\end{tabular}

* Assumes $p_{\infty}=1.0 \times 10^{7} \mathrm{~Pa}, \mu=2.1 \times 10^{-3} \mathrm{~Pa} \cdot \mathrm{s}$.

$\dagger$ Fit for $t<8.64 \times 10^{6} \mathrm{~s}(100 \mathrm{~d})$.

$\ddagger$ Fit for $t<1.04 \times 10^{7} \mathrm{~s}(120 \mathrm{~d})$.

idealized model can be taken, and where it begins to break down. A linear portion of the curve with positive slope is the region where the model may yield meaningful results. A departure from the linear trend at some point may indicate that phenomena not accounted for by the model, such as crack growth, started to influence the flow. Second, this method allows a local fit in those regions where it appears to apply; for example, data at very late time that become erratic can be excluded from the fit. Of course, this introduces considerable subjectivity into the fitting process, and the results should be viewed with this in mind. 
Note that the data for hole DBT15 were fit in this case as two independent sets. The data for the first stage, DBT15A, were fit for properties representative of the salt penetrated by the initial length of the hole. For the purpose of fitting the second stage following elongation, DBT15B, all brine collected was assumed to be derived from the new length of hole. That is, for the late-time analysis, the contribution of the initial hole length to the brine collected during the second stage was considered negligible.

\subsubsection{Results}

Figures 6-27 through 6-40 show all data plotted as $|q|^{-1}$ versus $\ln t$ and the linear fits found for selected portions of the data. The resulting hydraulic properties are given in Table 6-3. Again, the permeability values are more reliable than the capacitance values due to limitations discussed previously. This fact is borne out by the relatively narrow range of permeability inferred, and the reasonable agreement with results from the more complete fits given in Tables 6-1 and 6-2. The permeabilities inferred here are again of the order of $10^{-22}$ to $10^{-21} \mathrm{~m}^{2}$, with one (QPB02) of order $10^{-20} \mathrm{~m}^{2}$. Most are within a factor of 2 of the values obtained by fitting the entire flux or cumulative volume histories.

The diffusivities or capacitances inferred by this method must be regarded as questionable. The latter fall in a range of order $10^{-12}$ to $10^{-10} \mathrm{~Pa}^{-1}$, with one (QPB02) value of order $10^{-9} \mathrm{~Pa}^{-1}$, and DBT12 giving $C=8.5 \times 10^{-16} \mathrm{~Pa}^{-1}$. The latter value is clearly absurd, as it implies compressibilities many orders of magnitude less than can be rationalized for the salt and brine (see Section 7.6). The extremely small capacitance inferred in this case results from the very slow decay rate observed at late time.

Notably, the capacitances inferred from these fits are, in many cases, about an order of magnitude smaller than those from the full fits. This occurs because the full data for many holes exhibit fluxes that drop off very slowly at very late time. Thus, the fits to the full data sets arrive at a relatively large value of $C$ in order that the characteristic time for the decay of the flux be large. In contrast, because the fits of the asymptotic expression (Equation 43) typically exclude the very late time data, when the linearity of $|q|^{-1}$ versus $\ln t$ appears to break down, intermediate times (typically centered around $\sim 10^{7} \mathrm{~s}$ ) are given more weight. During the period when the asymptotic expression appears to represent the data, the decay of the flux occurs at a somewhat higher rate than that inferred for the full histories using Equations (35) or (36), and this is reflected in smaller inferred values of $C$. 


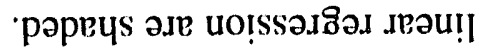

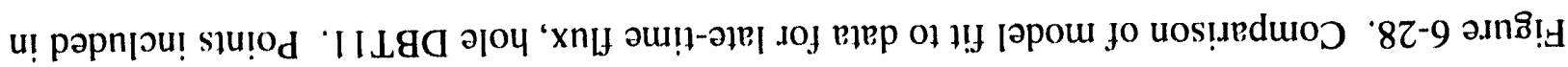
(1) 81)8: 111191811

(s) $\partial w ! \perp$

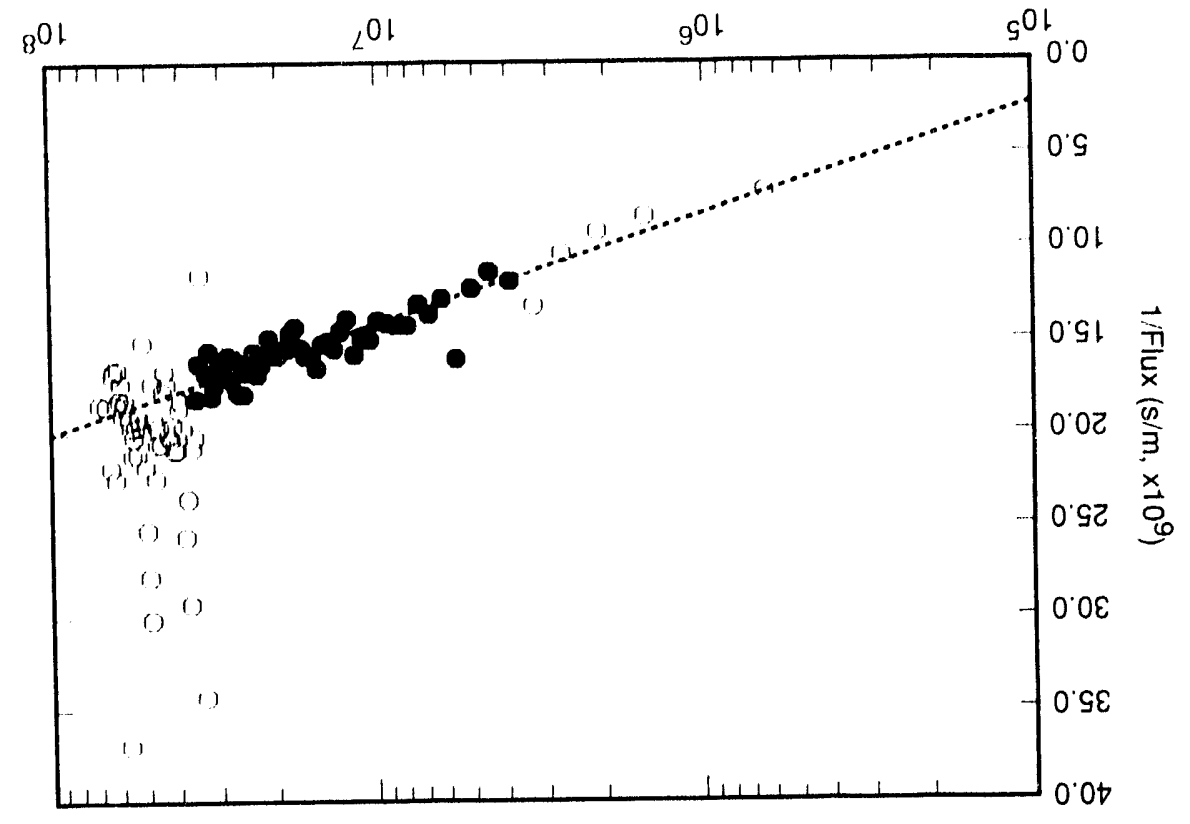

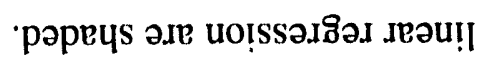

u! pəpnןou! słu!̣ $0 \angle 0 E 6119 \mid t \perp$

(s) อu!!

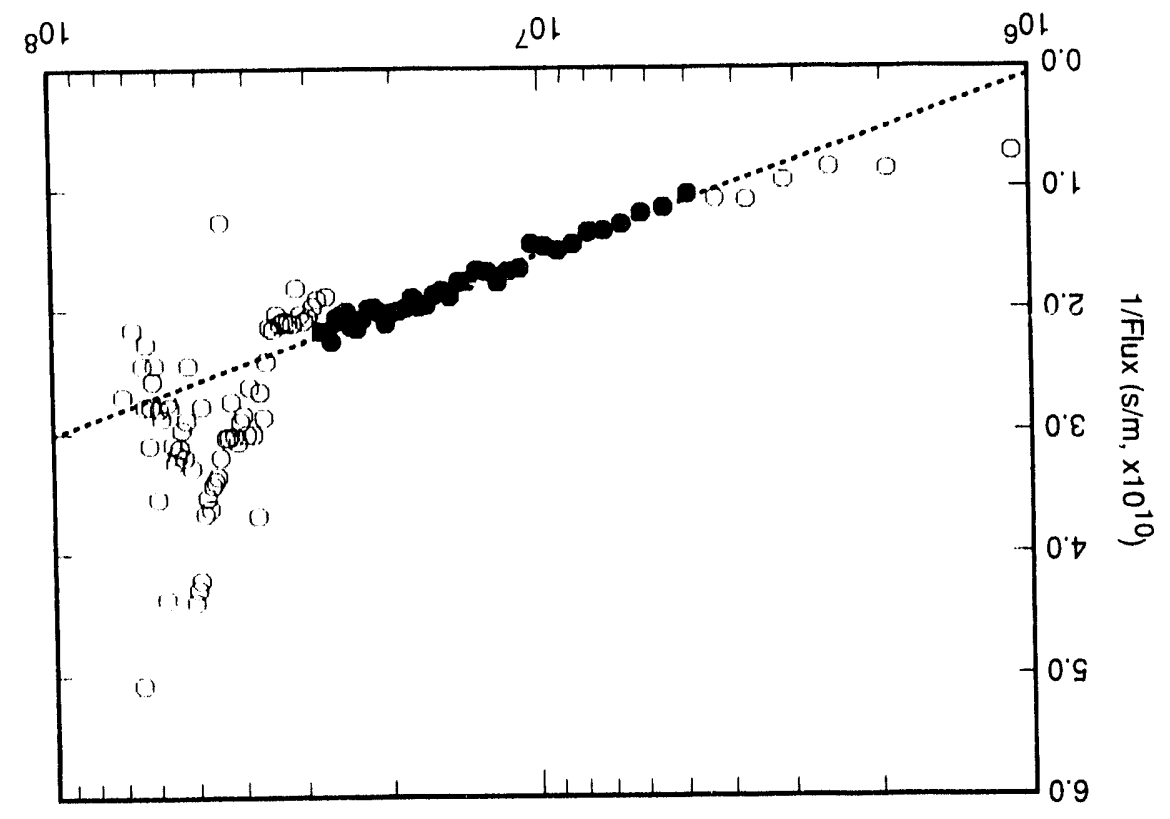




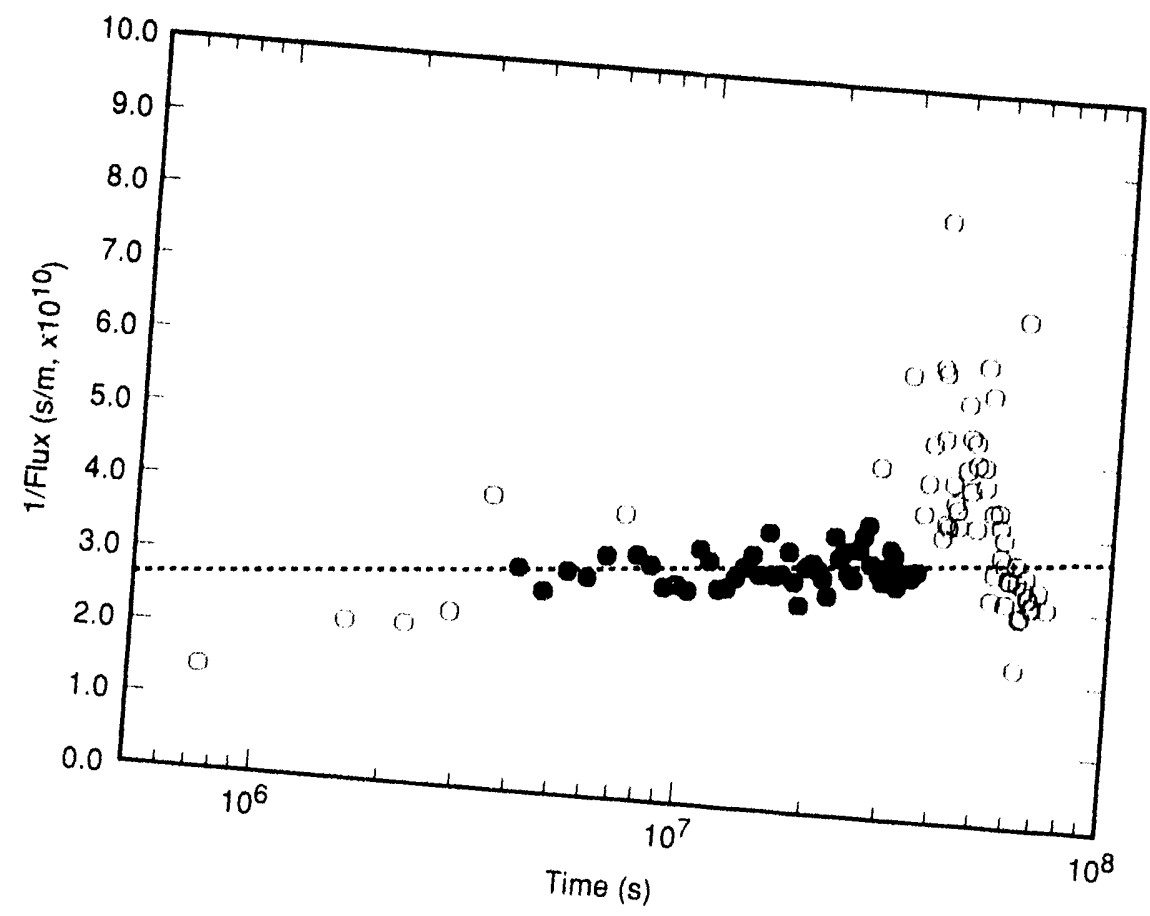

Figure 6-29. Comparison of model fit to data for late-time fluX, hole DBT1919.309.0
TR1. linear regression are shaded.

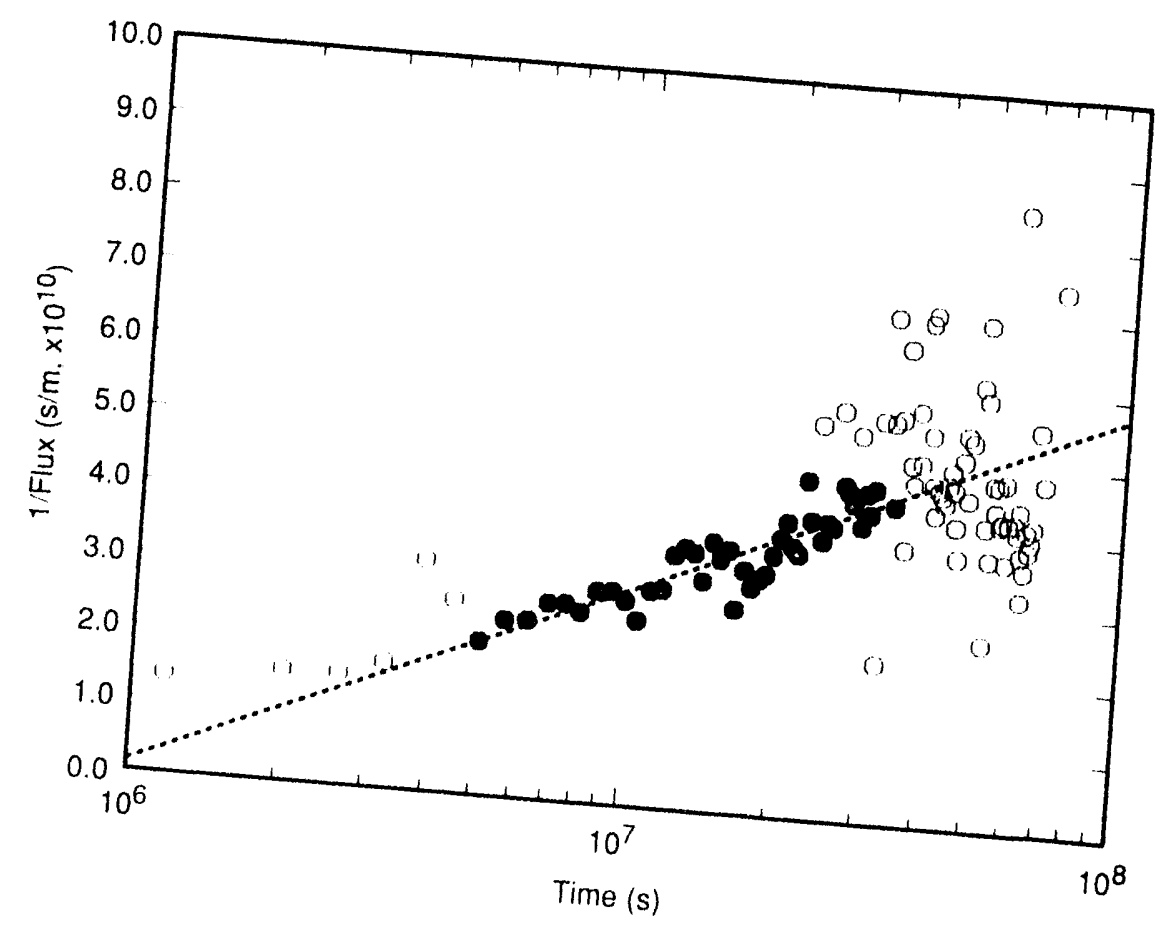

Figure 6-30. Comparison of model fit to data for late-time flux, hole DRT1919.310.0 linear regression are shaded. 


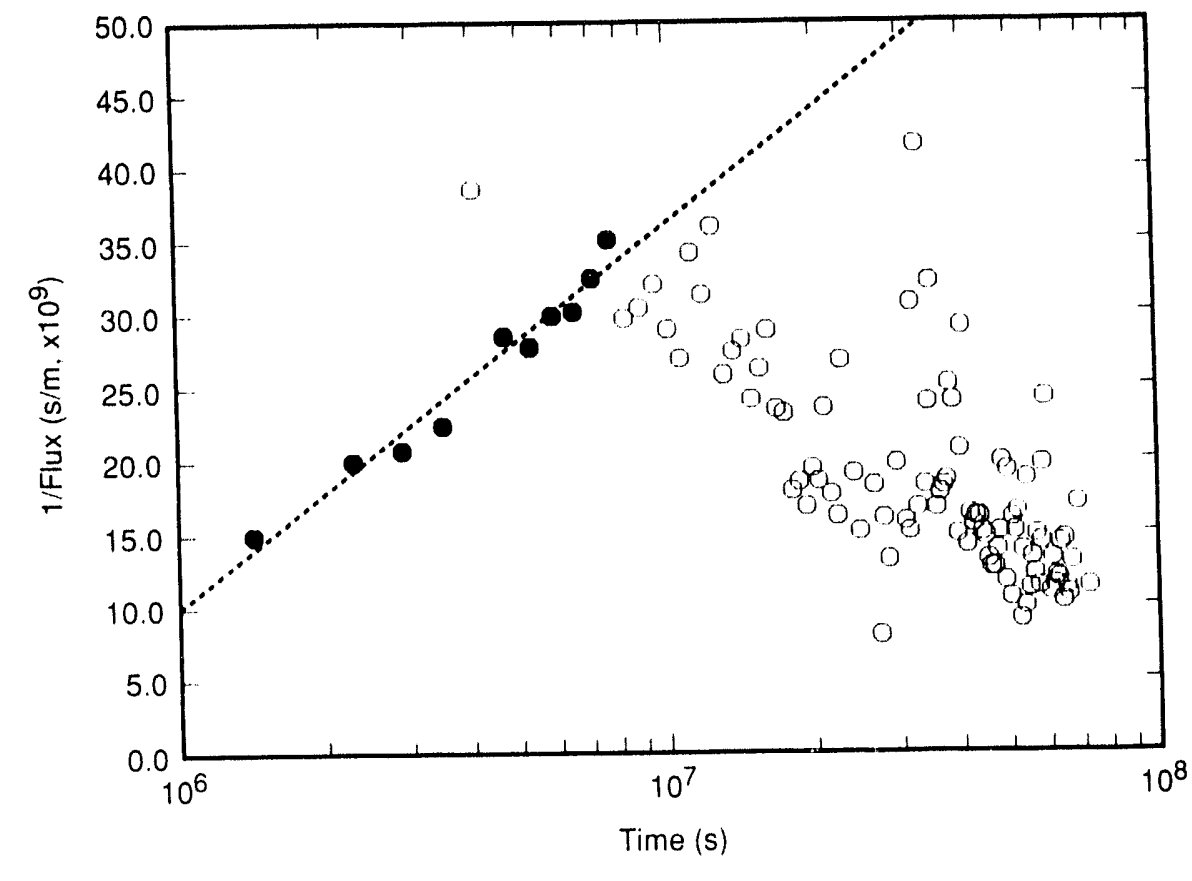

Figure 6-31. Comparison of model fit to data for late-time flux, hole DBT14A. Points included in linear regression are shaded.

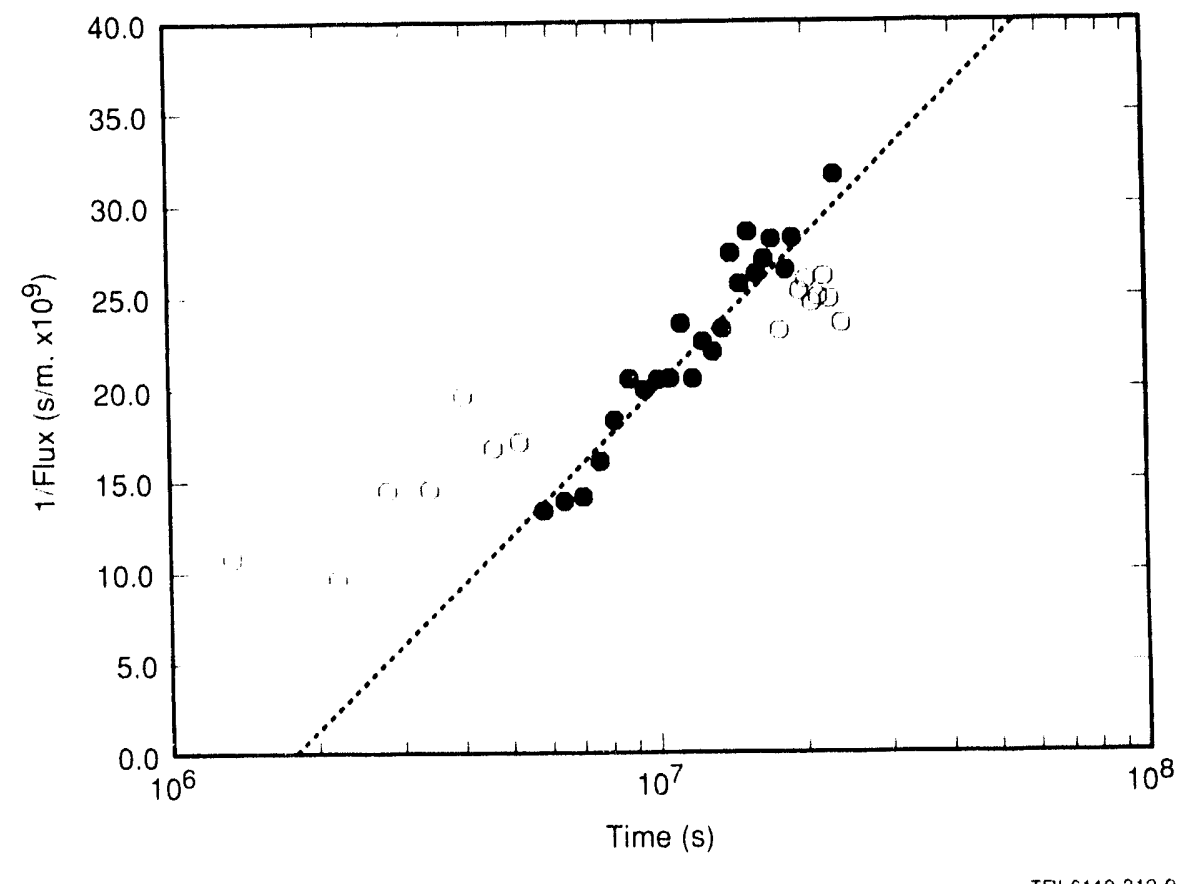

Figure 6-32. Comparison of model fit to data for late-time flux, hole DBT15A. Points included in linear regression are shaded. 


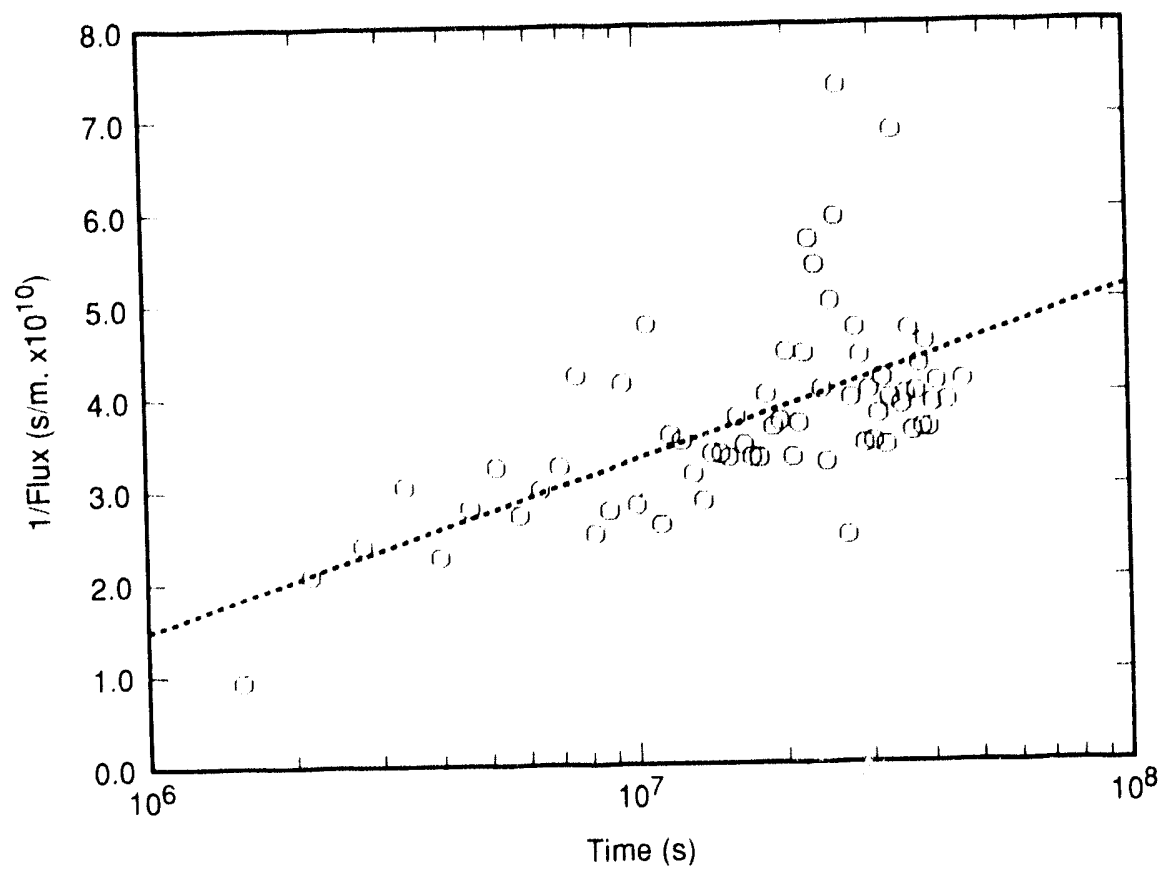

TRI. $6119 \cdot 313 \cdot 0$

Figure 6-33. Comparison of model fit to data for late-time flux, hole DBT15B. All points shown were included in linear regression.

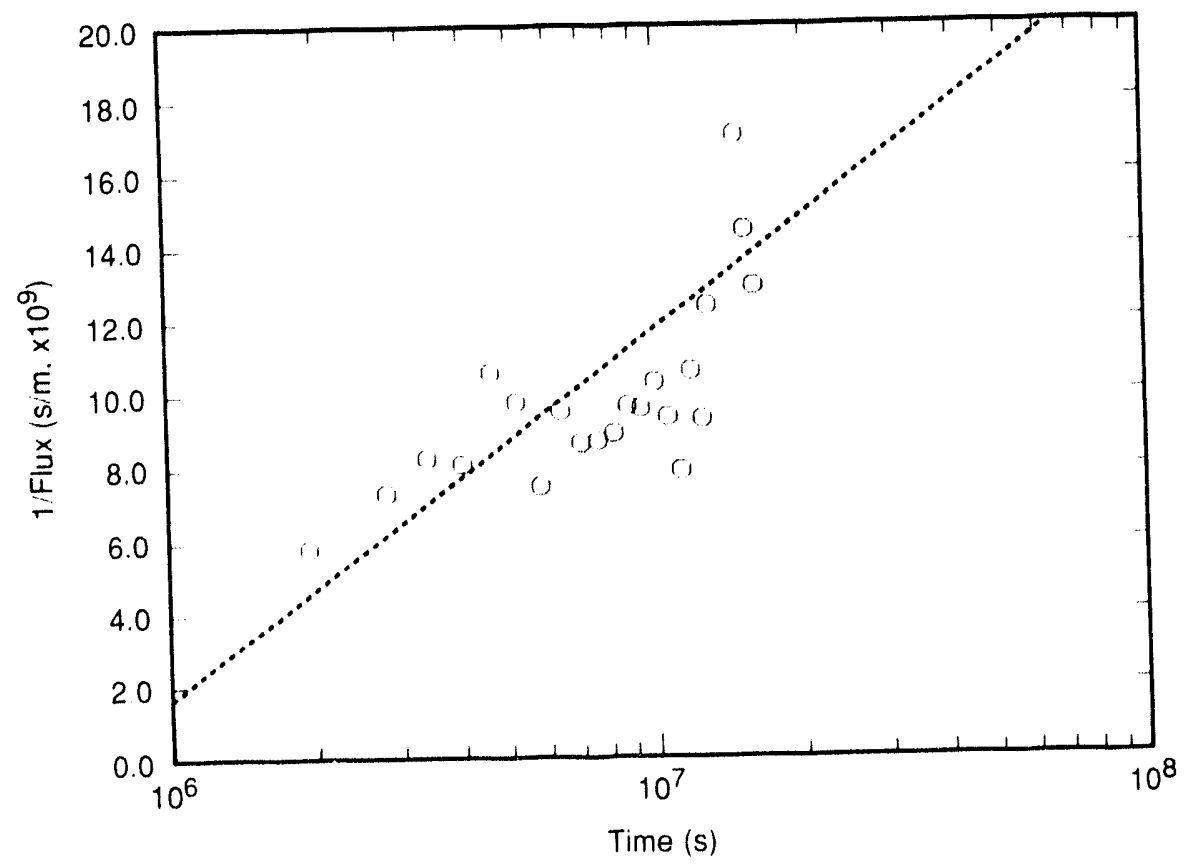

TRI 61193140

Figure 6-34. Comparison of model fit to data for late-time flux, hole DBT31A. All points shown were included in linear regression. 


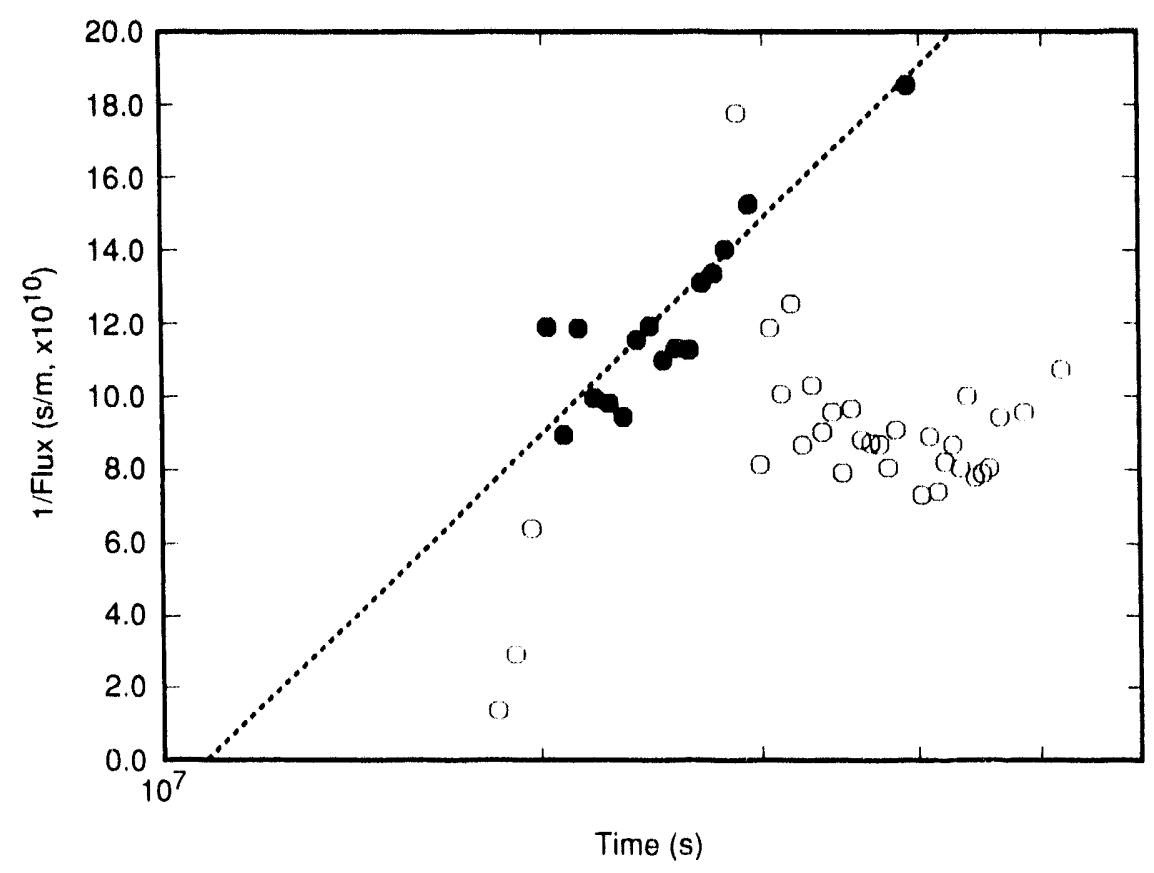

TRI. 6119.3150

Figure 6-35. Comparison of model fit to data for late-time flux, hole DBT32C. Points included in linear regression are shaded.

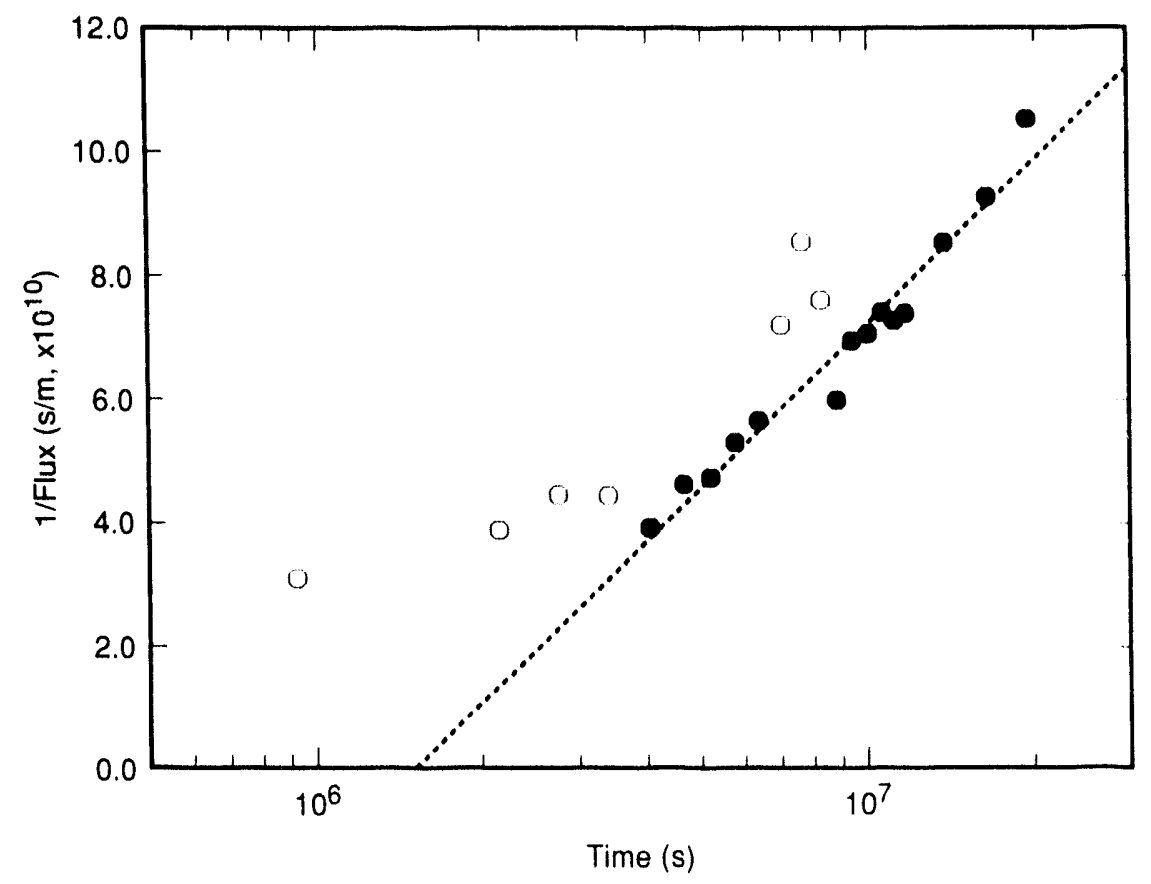

TRI $6119 \cdot 316 \cdot 0$

Figure 6-36. Comparison of model fit to data for late-time flux, hole L4B01. Points included in linear regression are shaded. 


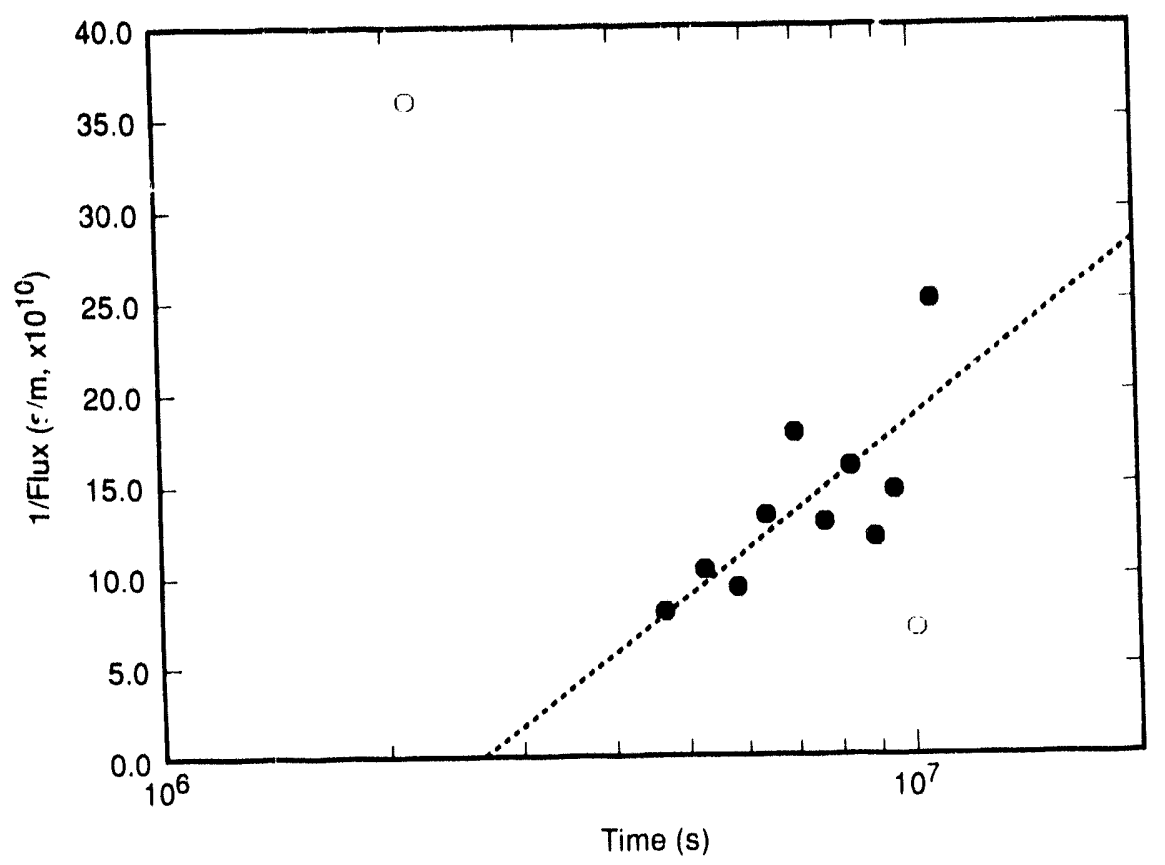

TP1. $6119 \cdot 317 \cdot 0$

Figure 6-37. Comparison of model fit to data for late-time flux, hole L4X01. Points included in linear regression are shaded.

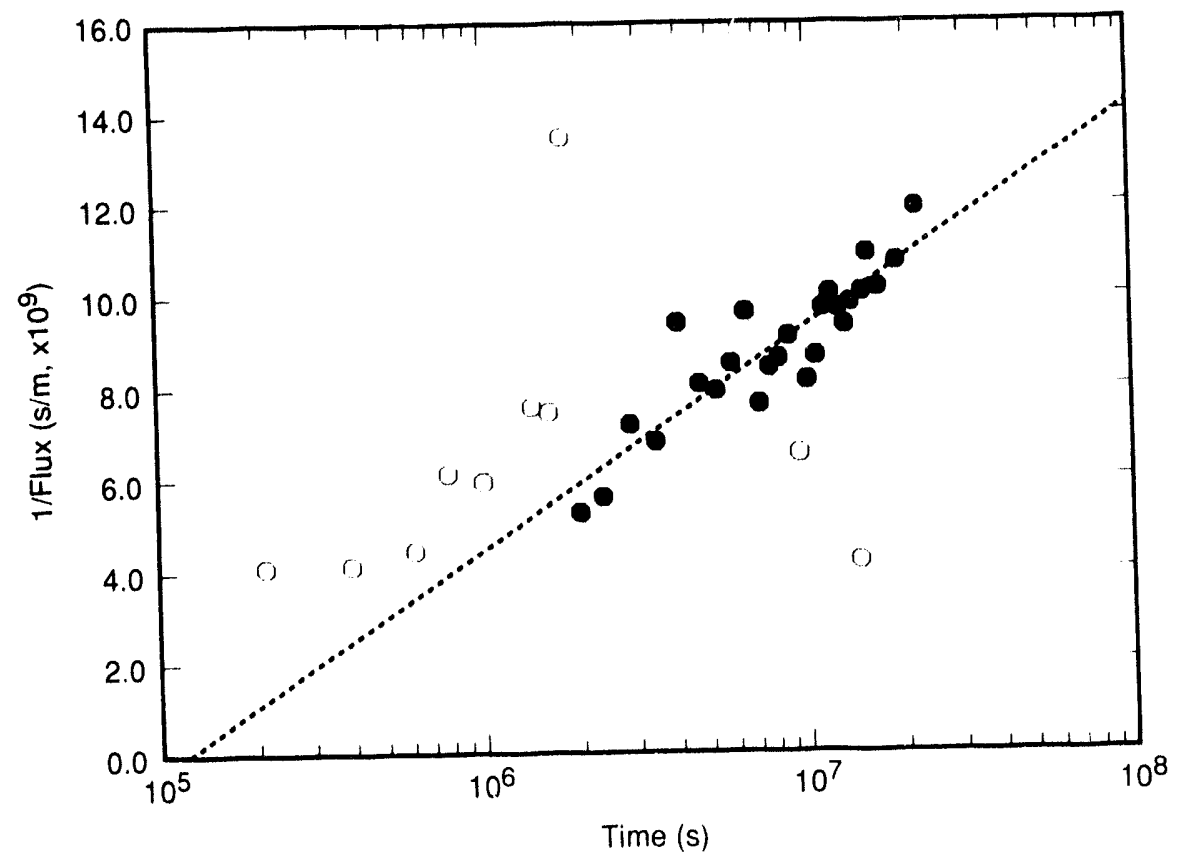

TRI 6119318.0

Figure 6-38. Comparison of nodel fit to data for late-time flux, hole QPB01. Points included in linear regression are shaded. 


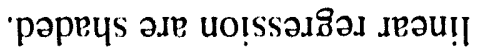

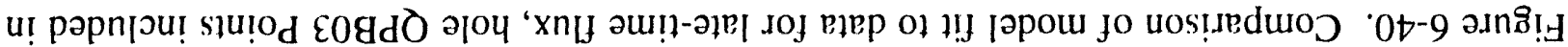

$0.028 .6119 \cdot 141$

(s) $\theta u ! \perp$

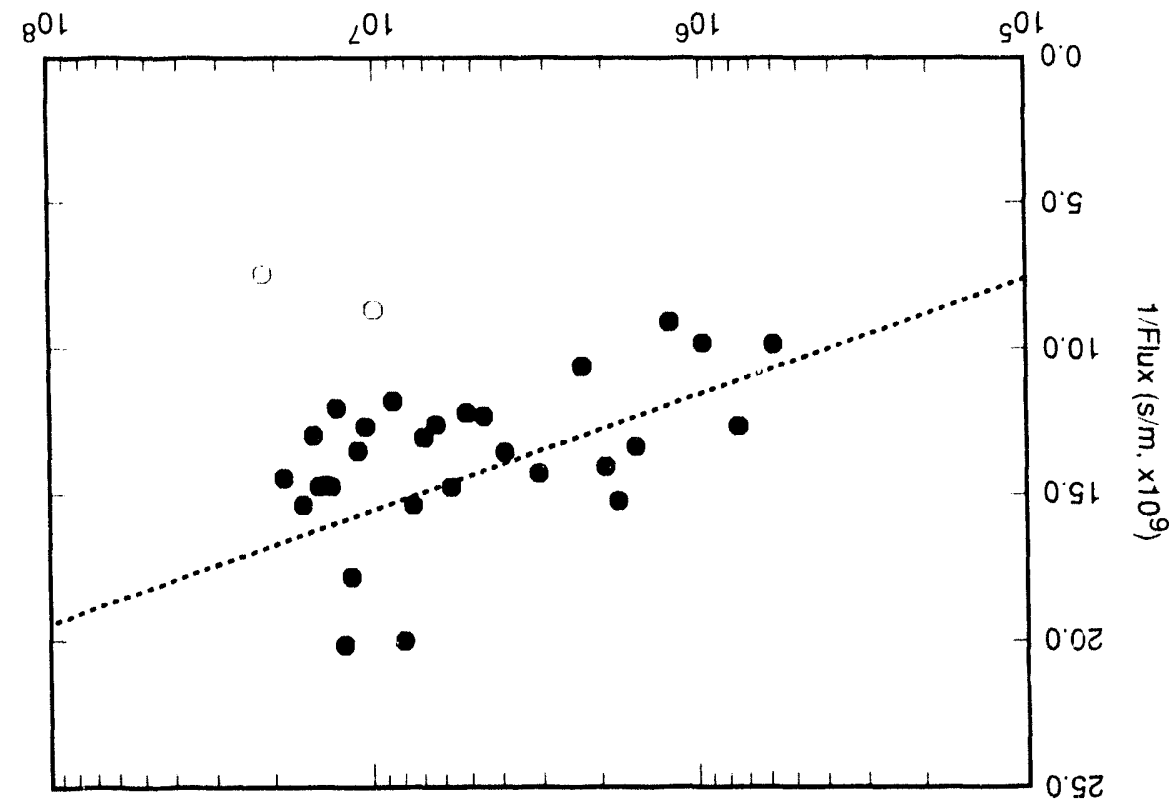

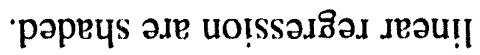

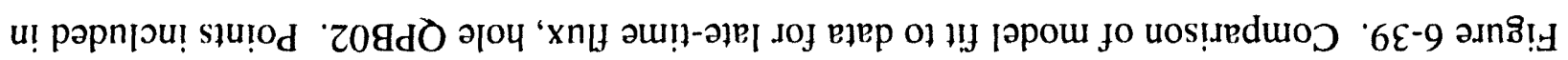
0.6186119 .181

(s) จu!!

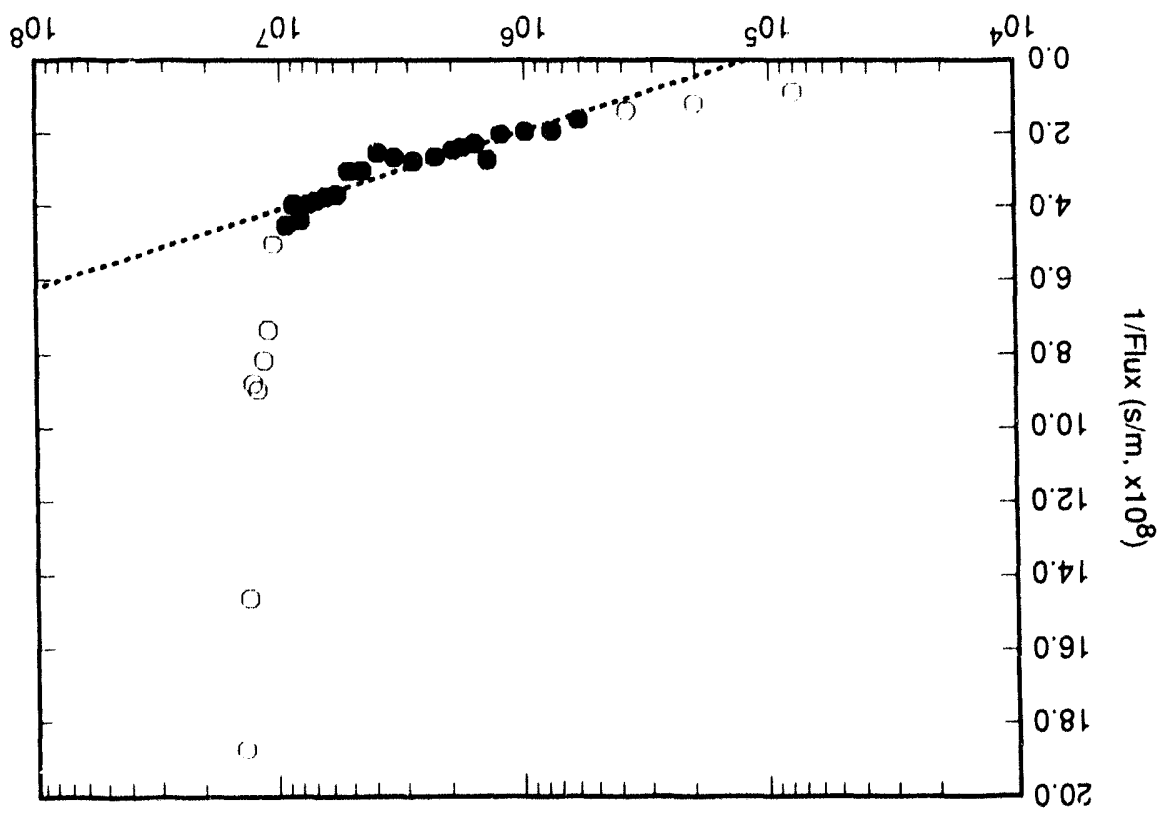


Table 6-3. Parameters Derived from Fits to Late-Time Flux

\begin{tabular}{|l|c|c|c|c|}
\hline \hline Hole & $\begin{array}{c}\text { Perm. } \times \text { Press. } \\
k_{p_{\infty}} \\
\left(\mathrm{m}^{2} \mathrm{~Pa}, \times 10^{-15}\right)\end{array}$ & $\begin{array}{c}\text { Permeability* } \\
k\end{array}$ & $\begin{array}{c}\text { Diffusivity } \\
\left(\mathrm{m}^{2}, \times 10^{-21}\right)\end{array}$ & $\begin{array}{c}\text { Capacitance* } \\
\left(\mathrm{m}^{2} / \mathrm{s}, \times 10^{-10}\right)\end{array}$ \\
$\left(\mathrm{Pa}^{-1}, \times 10^{-9}\right)$ \\
\hline DBT10 & 8.33 & 0.833 & 22.8 & 0.174 \\
DBT11 & 20.7 & 20.7 & 508 & 0.0195 \\
DBT12 & 26.0 & 26.0 & $1.45 \times 10^{7}$ & $8.53 \times 10^{7}$ \\
DBT13 & 4.38 & 4.38 & 22.8 & 0.0914 \\
DBT14A & 4.72 & 4.72 & 49.4 & 0.0455 \\
DBT14B & - & - & - & - \\
DBT15A & 4.58 & 0.458 & 11.5 & 189 \\
DBT15B & 12.8 & 1.28 & 133 & 0.0457 \\
DBT31A & 12.2 & 1.22 & 29.8 & 0.195 \\
DBT32C & 3.31 & 0.331 & 154 & 0.0102 \\
L4B01 & 1.40 & 0.140 & 13.6 & 0.0492 \\
L4X01 & 3.44 & 0.344 & 626 & 0.00261 \\
QPB01 & 12.6 & 1.26 & 42.6 & 0.141 \\
QPB02 & 287 & 28.7 & 41.3 & 3.31 \\
QPB03 & 15.6 & 1.56 & 4300 & 0.00172 \\
QPB04 & - & - & - & - \\
QPB05 & - & - & -- & - \\
\hline \hline
\end{tabular}

* Assumes $p_{\infty}=1.0 \times 10^{7} \mathrm{~Pa}, \mu=2.1 \times 10^{-3} \mathrm{~Pa} \cdot \mathrm{s}$. 


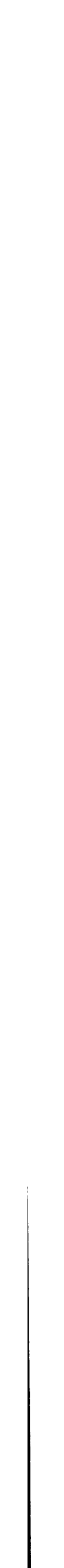

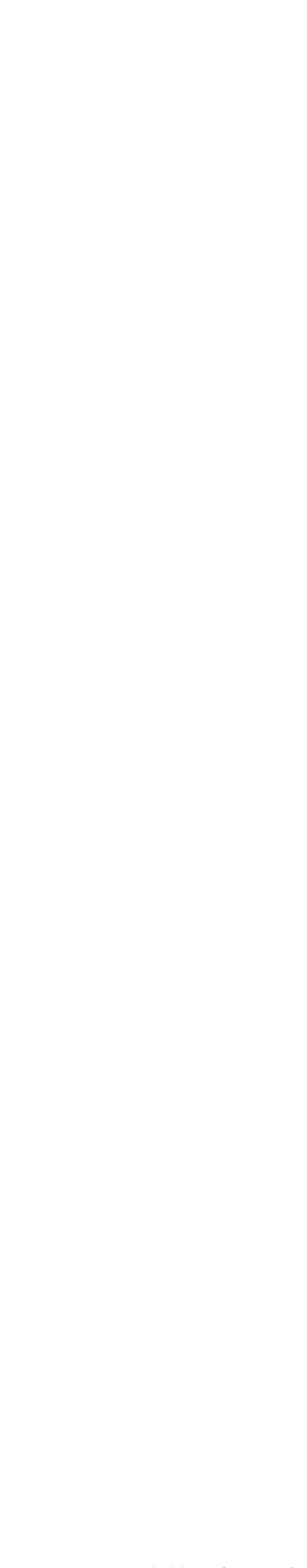




\subsection{SUMMARY AND DISCUSSION}

Data from the small-scale brine inflow experiments for the period to January 1990 have been reduced and analyzed. The permeability and hydraulic diffusivity of the salt have been estimated by fitting an idealized model to the data. The model assumes that brine seepage to the monitored boreholes is due to relaxation of ambient pore-water pressure by darcy flow. It is further assumed that the salt is isotropic and homogeneous around each hole, the initial pressure is uniform, and the flow is normal to the hole axes. An exact, analytical solution is available for the linear diffusion problem corresponding to this corifiguration. The solution has been fit to the data for each hole by a least-squares method, which yields estimates of two parameters for each fit. The two parameters scale the magnitude of the flow and the time scale over which it evolves. With an additional assumption for the initial brine pressure, the permeability and diffusivity can be extracted.

Three fitting schemes have been applied, entailing fits to the brine flux, the cumulative brine volume, and the brine flux at "late" time. For those data consistent with the model prediction, estimated permeabilities are typically $10^{-22}$ to $10^{-21} \mathrm{~m}^{2}$. The relatively small range of permeabilities inferred reflects the observation that the observed seepage fluxes are fairly consistent from hole to hole, of the order of $10^{-10} \mathrm{~m} / \mathrm{s}$. Estimated diffusivities are typically $10^{-10}$ to $10^{-8}$ $\mathrm{m}^{2} / \mathrm{s}$. The great scatter in inferred hydraulic diffusivities is due to the difficulty of matching the idealized model history to the observed evolution of the flows. The data obtained from several of the monitored holes are not consistent with the simple model adopted here; material properties could not be inferred in these cases.

The results of the fits to the full flux histories are displayed graphically in Figures 7-1 through 7-3 as histograms of the permeability, hydraulic diffusivity, and capacitance, respectively. A total of 15 fits are available (see Table 6-1). The histograms are constructed with logarithmic scales for the properties. The "bins" used to construct the histograms are bounded by $(0.5-5.0) \times 10^{\text {n }}$, where $10^{\mathrm{n}}$ is plotted at the center of each bar. The logarithmic mean of each of the three properties is:

$$
\begin{aligned}
& \bar{k}=0.48 \times 10^{-21} \mathrm{~m}^{2}, \\
& \bar{c}=5.34 \times 10^{-10} \mathrm{~m}^{2} / \mathrm{s}, \\
& \bar{C}=0.43 \times 10^{-9} \mathrm{~Pa}^{-1},
\end{aligned}
$$

The range spanning plus or minus one standard deviation of the logarithm of each parameter is:

$$
\begin{gathered}
\bar{k}=(0.056-4.1) \times 10^{-21} \mathrm{~m}^{2}, \\
\bar{c}=(0.075-380) \times 10^{-10} \mathrm{~m}^{2} / \mathrm{s}, \\
\bar{C}=(0.029-6.4) \times 10^{-9} \mathrm{~Pa}^{-1},
\end{gathered}
$$




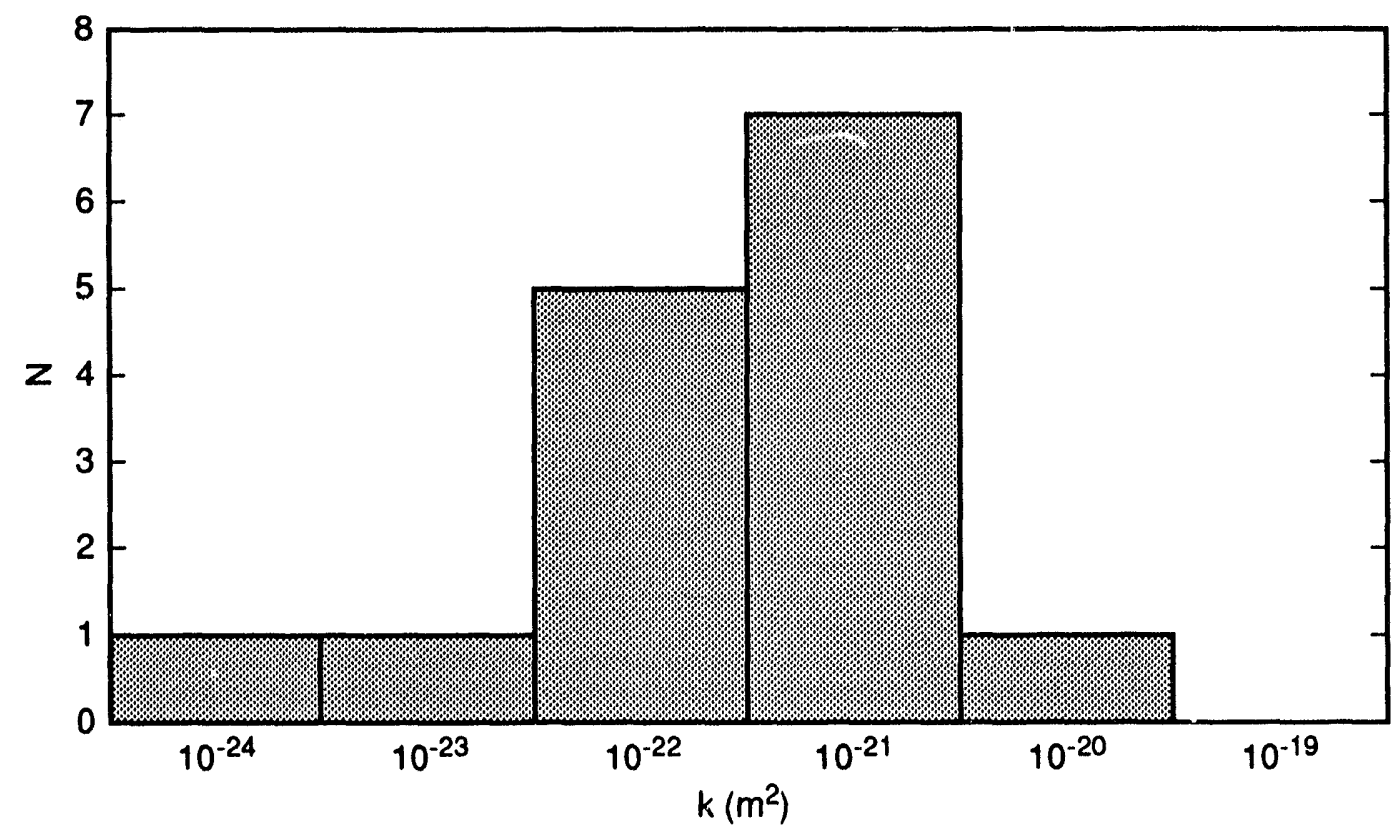

TRI-61 19-321-0

Figure 7-1. Histogram of permeabilities; fits to full flux histories (Table 6-1). Bins centered on $10^{\mathrm{n}}$ include values from 0.5 to $5.0 \times 10^{\mathrm{n}}$. Mean value of $\log k$ is -21.3 ; standard deviation of $\log k$ is 0.9 .

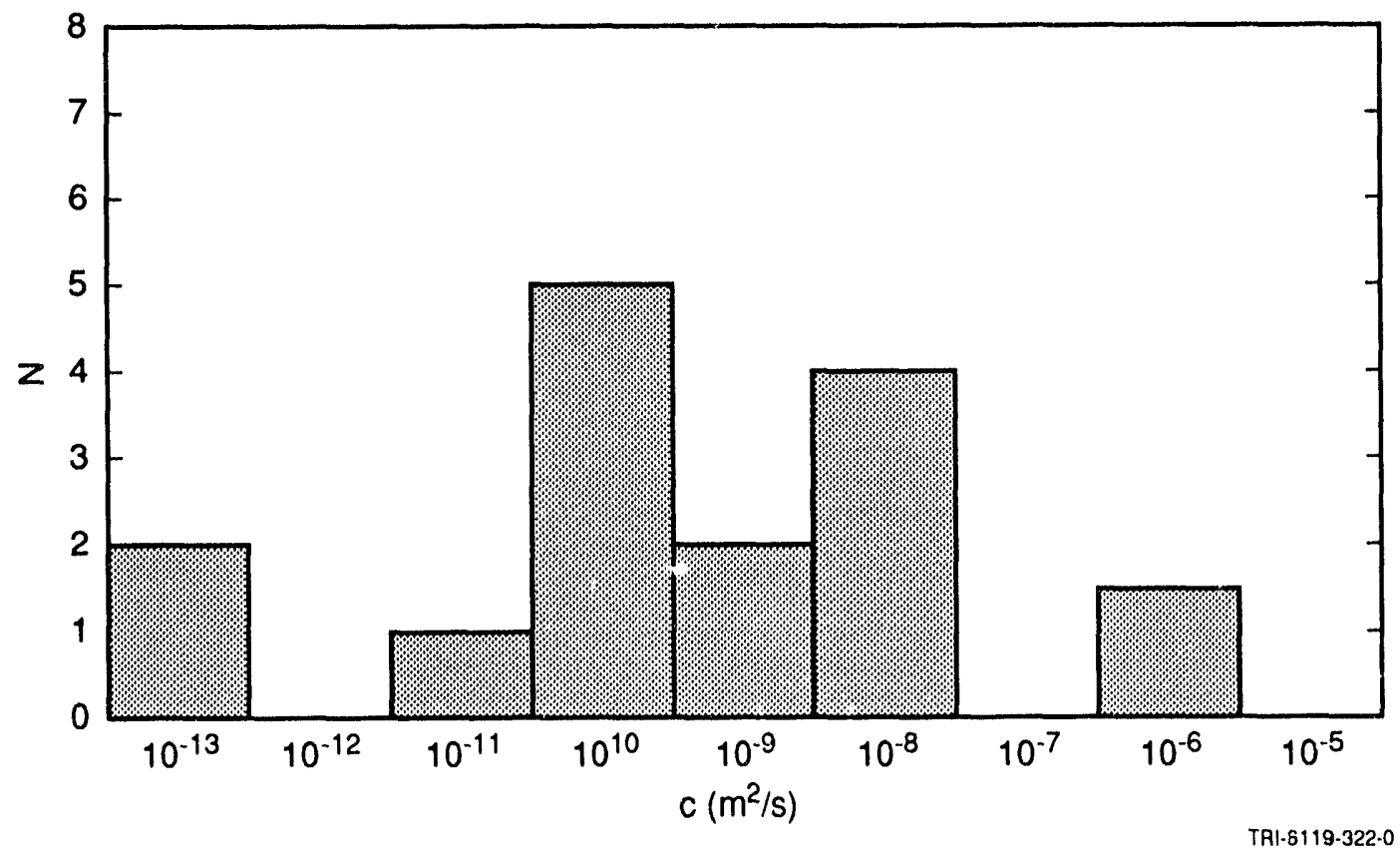

Figure 7-2. Histogram of hydraulic diffusivities; fits to full flux histories (Table 6-1). Bins centered on $10^{\mathrm{n}}$ include values from 0.5 to $5.0 \times 10^{\mathrm{n}}$. Mean value of $\log c$ is -9.3 ; standard deviation of $\log c$ is 1.8 . 


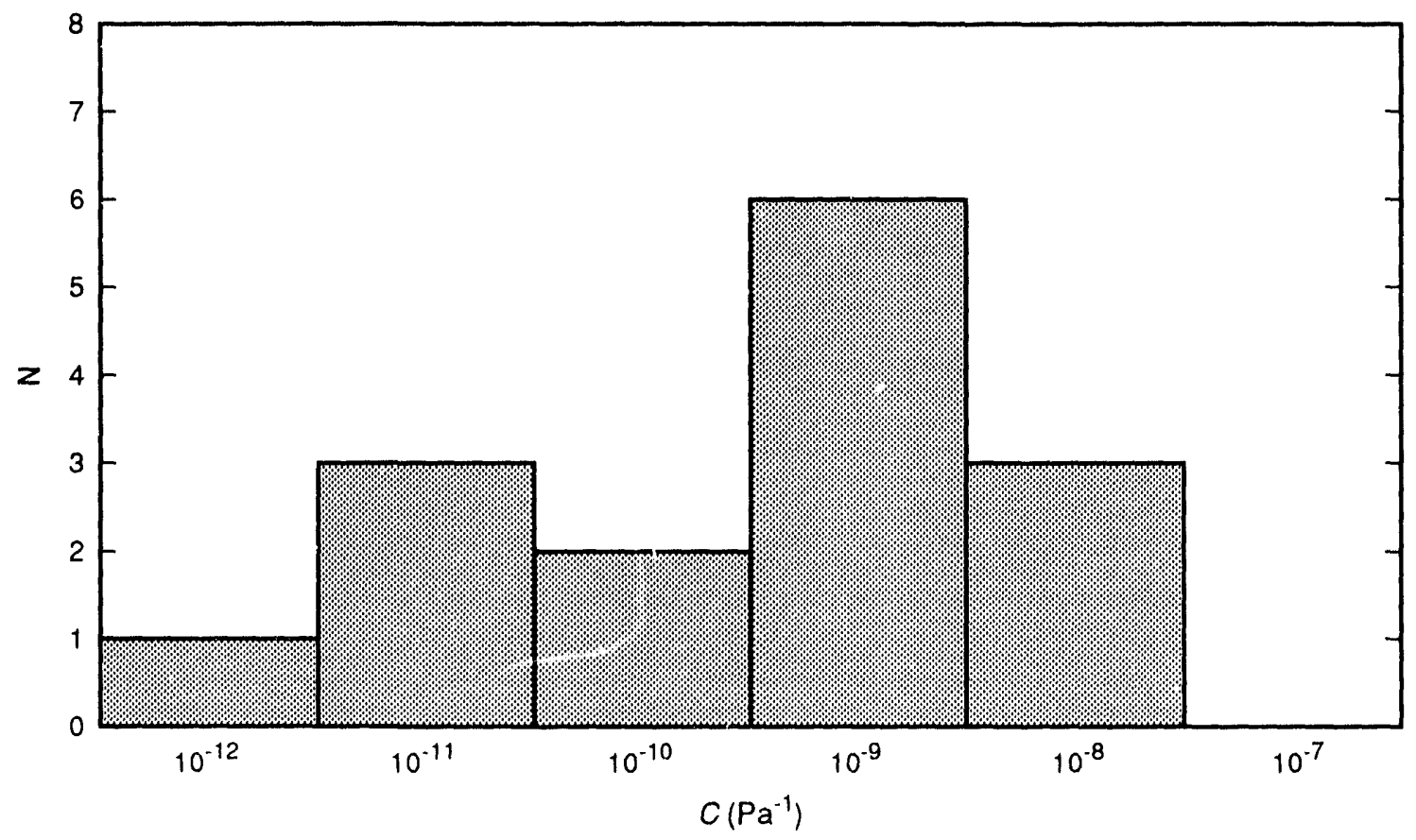

TRI.6119.323.0

Figure 7-3. Histogram of capacitances; fits to full flux histories (Table 6-1). Bins centered on $10^{\mathrm{n}}$ include values from 0.5 to $5.0 \times 10^{\mathrm{n}}$. Mean value of $\log C$ is -9.4 ; standard deviation of $\log C$ is 1.2 .

The present data are too sparse to draw conclusions regarding the statistical distributions of the properties. Notably, it is sometimes claimed that permeability is log-normally distributed within a given geological formation (Freeze and Cherry, 1979). Figure 7-1 does not contradict this claim. Freeze (1975) found that the standard deviation of the logarithm of permeability typically falls in the range 0.5 to 1.5 . The standard deviation of the logarithms of the 15 permeability determinations shown in Figure 7-1 is 0.9 , in remarkable agreement with the observation of Freeze (1975), based on much more extensive data sets. Thus, the scatter in the permeabilities inferred in this study appears to be typical of geologic media.

It should be noted that the boreholes in the Room Q Access Drift, QPB01 through 05, all penetrate Marker Bed 139, a horizon known to produce relatively large quantities of brine (Finley et al., 1992). The average thickness of the marker bed is about $0.9 \mathrm{~m}$; the QPB holes are $3.1 \mathrm{~m}$ long. Therefore, the brine inflow to these holes may be interpreted as being dominated by the marker bed, and the fits as characterizing the properties of the marker bed. In this case, according to the discussion in Section 5.5, both the permeabilities and the capacitances given in Tables 6-1, 6-2 and 6-3 should be multiplied by a factor of 3.4. 
Some of the limitations of the idealized, one-dimensional, unbounded, radial flow model used here are discussed in the reports by Webb (1992) and Gelbard (1992).

\subsection{Choice of Parameters for the Fits}

The regressions reported here are two-parameter fits. For example, as noted in Section 6.1, the flux is fit for a magnitude, $q_{0}=k p_{\infty} / \mu a$, and a characteristic decay time, $t_{0}=a^{2} / c$. The approach followed in the foregoing was to treat the borehole radius, $a$, and the brine viscosity, $\mu$, as known, because they can be determined with good accuracy by direct, independent measurements. The initial brine pressure was also treated as known, with the constant value $p_{\infty}=$ $10 \mathrm{MPa}$ assumed. This leaves the permeability, $k$, and the diffusivity, $c$, as unknowns determined by the fits. The capacitance, $C=k / \mu c$, can then be computed as well.

An alternative scheme that can be applied in a system whose mechanical properties are well characterized is to consider the capacitance known. This requires a model for the relationship between capacitance and various material properties (e.g., Equation 15), as well as independent laboratory measurements of the rock and fluid compressibilities. In this case, the two remaining unknowns determined by the fits are the permeability and the initial pressure, $p_{\infty}$. This scheme is attractive because $p_{\infty}$ is indeed unknown, can be expected to exhibit some variability, and can be determined only by in situ measurements. However, its success rests on the assumption that the storage mechanisms are well understood, properly represented by the model adopted, and characterized accurately by the independent measurements. That this is not the case for the salt is suggested by the following argument. The field data for borehole seepage considered in this report indicate a relatively long time scale that characterizes the decay of the flux, typically 100 days. The long time scale implies that the diffusivity is small, which, for fixed capacitance, implies that the permeability is small. In order to match the magnitude of the flux given a small permeability, the inferrred formation pressure must be unrealistically large.

A sample calculation illustrates this point. Suppose that $t_{0}$ is $10^{7} \mathrm{~s}(\sim 116$ days); assume that $C=1.0 \times 10^{-11} \mathrm{~Pa}^{-1}$, which is consistent with various estimates based on the elastic properties of the salt and brine (Freeze and Cherry, 1979; Nowak and McTique, 1987). Then, with $a=0.05 \mathrm{~m}$ and $\mu=0.0021 \mathrm{~Pa} \cdot \mathrm{s}$, the inferred permeability is $k \sim a^{2} \mu \mathrm{C} / t_{0} \simeq 5 \times 10^{-24} \mathrm{~m}^{2}$. That gives (with $q_{0} \simeq 10^{-10} \mathrm{~m} / \mathrm{s}$ ), $p_{\infty} \sim q_{0} \mu a / k \simeq 2 \times 10^{9} \mathrm{~Pa}$, which is clearly absurd (the lithostatic stress is two 
orders of magnitude smaller). Thus, as discussed in the foregoing paragraph, this scheme leads to implausible estimates of both the permeability and the formation pressure.

The fitting scheme adopted in this report, (i.e., to regard the formation pressure as known and the permeability and capacitance as unknowns), in some sense acknowledges that the mechanisms affecting capacity or storage in the salt may not be understood fully. This uncertainty is then submerged in the estimation of the capacitance, which should be viewed as an "effective" or "apparent" property of the salt. It is important to recognize, however, that the large values of capacitance inferred from the fits (e.g., Table 6-1) are not easily reconciled with the classical models of an elastic, porous skeleton and compressible fluid (e.g., Equation 15). Such models predict a capacitance of the order of $C \sim 10^{-11} \mathrm{~Pa}^{-1}$, while the fits yielded many estimates of the order of $10^{-10}$ to $10^{-8} \mathrm{~Pa}^{-1}$. These estimates are discussed further in Section 7.5.

\subsection{Validity of the Radial Flow Approximation}

After a sufficiently long time, the measured flow to a borehole becomes sensitive to the finite length of the hole. A simple validity check for the one-dimensional, radial flow assumption is to compute the "diffusion" length for the duration of the test, and compare this to the length of the hole. The diffusion length is defined by $L_{d}=\sqrt{c t}$, and represents the characteristic radial distance to which the pressure relaxation has propagated after time $t$. If $L_{d} / L \ll<1$, the zone of relaxed pressure near the borehole is still highly elongate, and the one-dimensional model is appropriate. The data for the Room D holes cover a period up to about $t=7.5 \times 10^{7} \mathrm{~s}$, while those for Rooms $\mathrm{L} 4$ and $\mathrm{Q}$ cover a period up to about $t=2.5 \times 10^{7} \mathrm{~s}$. The corresponding diffusion lengths for various diffusivity values are shown in Table 7-1. Note that the criterion $L_{d} / L \ll 1$ is satisfied for the holes under consideration only if $c$ is $10^{-8} \mathrm{~m}^{2} / \mathrm{s}$ or less. The fits indicate that this condition holds for most of the fits for most of the holes.

The effect of the third dimension, (e.g., a finite-length hole), is typically to weaken the flow relative to the radial case. This is a consequence of geometric "spreading" of the diffusive front. For example, while the radial flux to a very long hole decays like $2 / \ln \left(4 t_{*} / C^{2}\right)$ at late time (Equation 41), the flux to a spherical cavity of radius $a$ decays (over its entire history) like

$$
q_{*}=1+\left(\pi t_{*}\right)^{-1 / 2},
$$


Table 7-1. Diffusion Length Scales for Various Diffusivities

\begin{tabular}{|c|c|c|}
\hline \multirow{2}{*}{ Diffusivity $\left(c, \mathrm{~m}^{2} / \mathrm{s}\right)$} & \multicolumn{2}{|c|}{ Diffusion Length $\left(L_{d}=\sqrt{c t}, \mathrm{~m}\right)$} \\
\cline { 2 - 3 } & $t=2.5 \times 10^{7} \mathrm{~s}$ & $t=7.5 \times 10^{7} \mathrm{~s}$ \\
\hline $10^{-10}$ & 0.050 & 0.087 \\
$10^{-9}$ & 0.158 & 0.274 \\
$10^{-8}$ & 0.500 & 0.866 \\
$10^{-7}$ & 1.58 & 2.74 \\
$10^{-6}$ & 5.00 & 8.66 \\
\hline
\end{tabular}

where the non-dimensionalizations are the same as those that were introduced in the cylindrical case (see Section 5). Thus, the effect of the third dimension is to allow the flux to decily more rapidly, and to approach a non-zero, steady-state value. If a particular data set is influenced by such geometric effects, but is fit by the radial flow model, the fit will tend toward small values of the diffusivity in order that the flux "hold up" over a long period of time.

Additional multidimensional effects are introduced by the presence of the room from which a borehole is drilled. First, there is seepage toward the mined faces of the room. Second, the initial mean stress state penetrated by a borehole is influenced by the room; the mean stress is reduced near mined faces, and the initial, undrained brine pressure is consequently reduced as well. Recent work by Gelbard (1992) considers both seepage toward the room wall and a depth-varying initial brine pressure in the context of the model discussed in this report. Again, both effects tend to weaken the brine inflow to a borehole. Neglect of these effects, as in the analysis presented in this report, results in underestimates of the permeability. Finally, creep of the salt results in a continued evolution of the mean stress field in the neighborhood of mined cavities, and this too is coupled to the brine flow. This effect is also neglected in the idealized analysis presented in this report.

\subsection{Comparison of Fits Over Various Time Intervals}

This section provides a comparison of parameters derived froni fits of both the flux and cumulative volume models to the same data over various time intervals. This exercise gives the reader a measure of the sensitivity of the results to various possible fitting schemes. Only data 
from DBT10 are treated; this is one of the "better-behaved" data sets, insofar as the response of the hole was quite smooth, and the rate of decay of the flux decreased monotonically. Results are summarized in Table 7-2. The variation in the inferred material properties reflects comments made previously in this report. The permeability is reasonably well constrained; all values fall within about a factor of 2 of $k \simeq 10^{-21} \mathrm{~m}^{2}$, whether from a fit to the flux or the volume, and also whether the data span 830,470 , or 230 days. The inferred diffusivity, however, is one to two orders of magnitude larger for the cumulative-volume fits, and it increases significantly for fits to the shorter history. Recall that the fits to the flux are more sensitive to the early-time response, so that the inclusion of more data has relatively little influence, here decreasing the inferred diffusivity by only a factor of 2 . In contrast, the fits to the volume are more sensitive to the later response, and the continually decreasing apparent diffusivity with longer data sets reflects the fact that the observed flow decayed more slowly than predicted by the idealized model at late time. That is, the characteristic time for the decay of the flux appears to be increasing with time. Therefore, a fit to a longer data set arrives at a smaller value of the diffusivity, $c$.

Table 7-2. Parameters for DBT10 by Various Fitting Schemes

\begin{tabular}{|l|c|c|c|c|}
\hline \hline \multicolumn{2}{|c|}{ Basis of fit } & $\begin{array}{c}\text { Diffusivity } \\
c\left(\mathrm{~m}^{2} / \mathrm{s}, \times 10^{-10}\right)\end{array}$ & $\begin{array}{c}\text { Permeability* } \\
k\left(\mathrm{~m}^{2}, \times 10^{-21}\right)\end{array}$ & $\begin{array}{c}\text { Capacitance* } \\
C\left(\mathrm{~Pa}^{-1}, \times 10^{-9}\right)\end{array}$ \\
\cline { 1 - 2 } Data type & Time period (d) & 0.54 & 0.41 & 3.6 \\
flux & 830 & 1.1 & 0.52 & 2.3 \\
flux & 470 & 1.1 & 0.54 & 2.3 \\
flux & 230 & 3.1 & 0.67 & 1.0 \\
cum. vol. & 830 & 17 & 1.1 & 0.31 \\
cum. vol. & 470 & 82 & 1.7 & 0.097 \\
cum. vol. & 230 & \multicolumn{2}{|c}{} & \\
\hline \hline
\end{tabular}

* Assumes $p_{\infty}=1.0 \times 10^{7} \mathrm{~Pa}, \mu=2.1 \times 10^{-3} \mathrm{~Pa} \cdot \mathrm{s}$.

These results emphasize that the permeability is fairly well constrained simply by the magnitude of the flows observed, while the diffusivity is more difficult to bound. Determination of the diffusivity depends on the details of the flow history, which was erratic for some holes. Furthermore, the flow histories are more likely to exhibit effects not accounted for by the idealized model for uniform, radial flow. 


\subsection{Comparison of Fits by Various Methods}

Figures 7-4 and 7-5 provide a graphical comparison of the variation in the parameters inferred by the three fitting procedures described in Section 6 and summarized in Tables 6-1, 6-2 and 6-3. In each case, the parameters inferred from fits based on the first method named in the legend are plotted as "Fit 1," and those from the second method are plotted as "Fit 2." Identical results from each method, of course, plot on a line with unit slope. Departures from this line indicate the discrepancies between methods. As noted previously, the fits for permeability generally result in reasonably consistent results, and no systematic variations are apparent in Figure 7-4. The inferred capacitances shown in Figure 7-5 exhibit larger differences, depending upon the fitting method. The plot shows that the fits based on the late-time-flux approximation tend to result in much smaller estimates of the capacitance. The reason is discussed in Section 6.4.

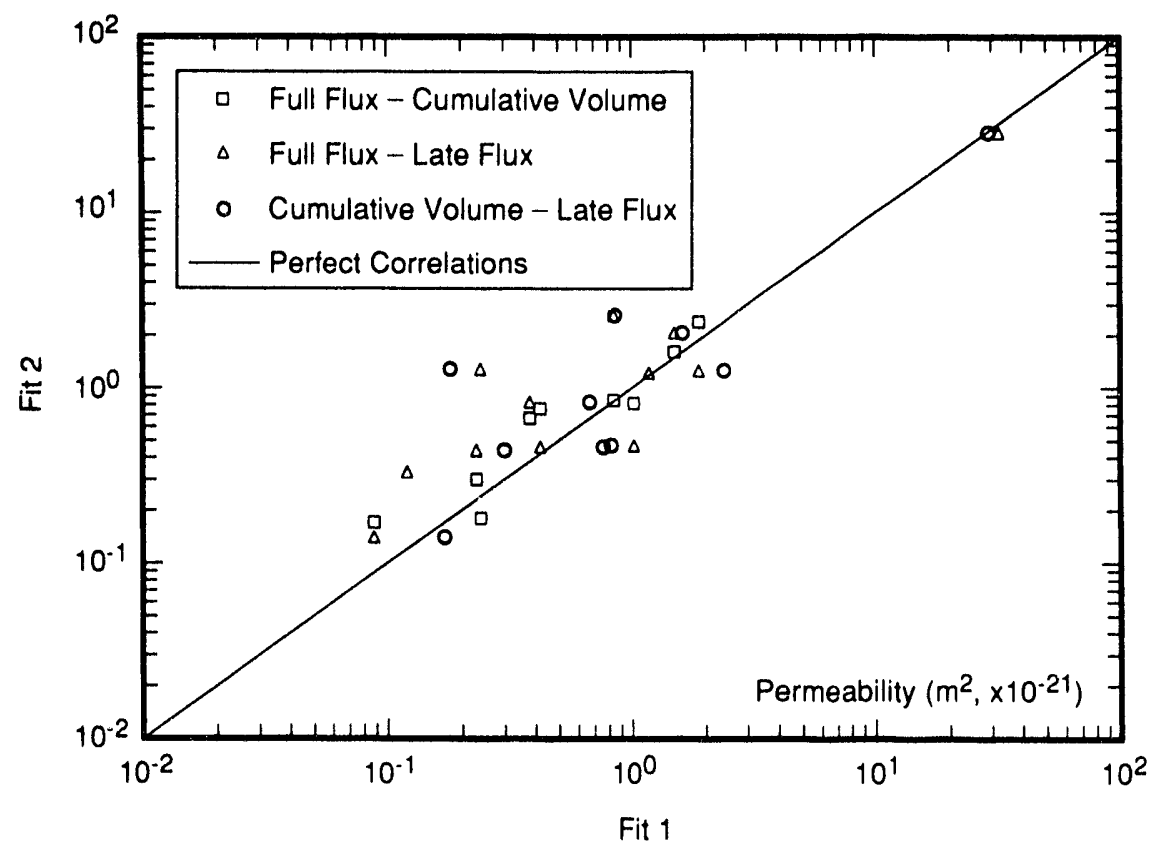

Figure 7-4. Comparison of permeability values $\left(\mathrm{m}^{2}, \times 10^{-21}\right)$ determined by various methods. Perfect correlations lie on the solid line. 


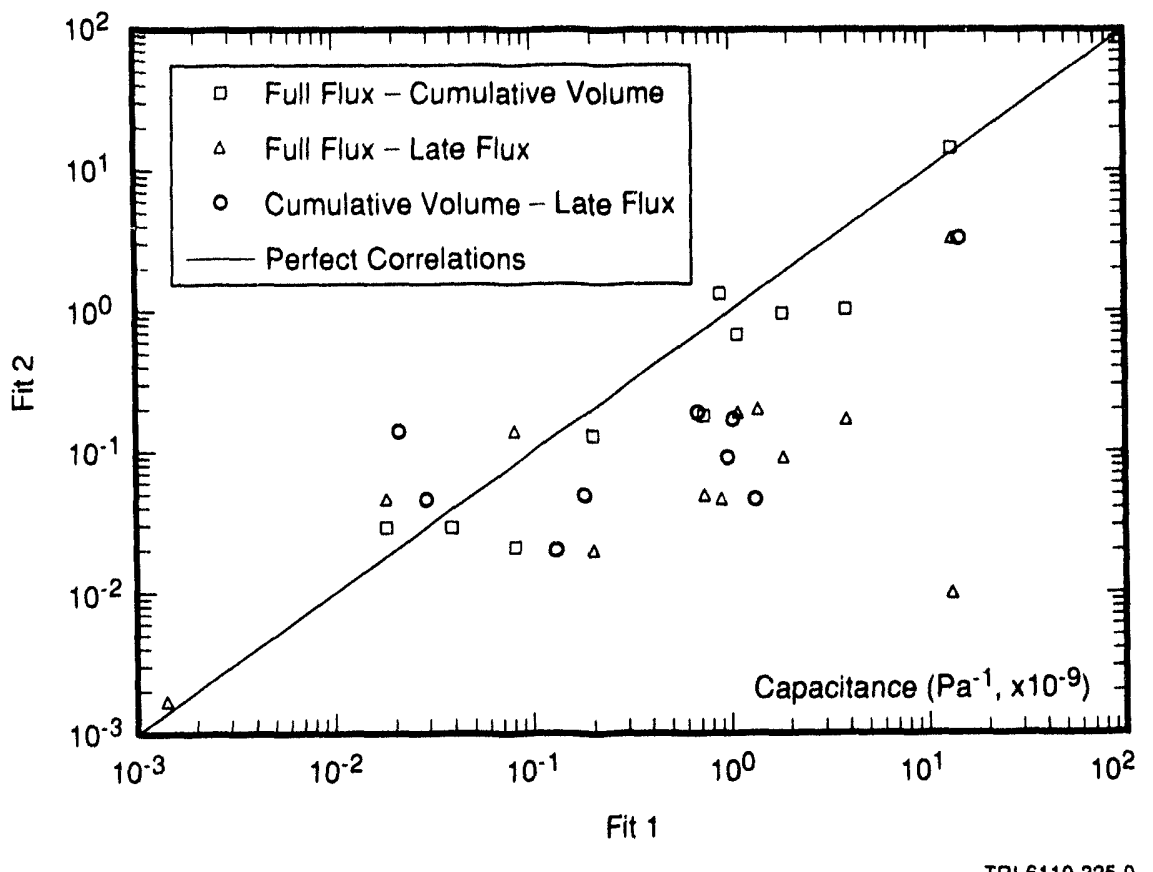

Figure 7-5. Comparison of capacitance values $\left(\mathrm{Pa}^{-1}, \times 10^{-9}\right)$ determined by various methods. Perfect correlations lie on the solid line.

\subsection{Additional Sources of Uncertainty}

\subsection{1 initial Brine Pressure}

The formation pressure was assumed to be $10 \mathrm{MPa}$ in order to separate the permeability. The error in the estimates of the permeability is proportional to the error in $p_{\infty}$. Various independent estimates of $p_{\infty}$ typically fall in the range of 0.5 to $12 \mathrm{MPa}$ (Beauheim et al., 1991). In general, it appears that pressures of about $10 \mathrm{MPa}$ are found for measurements taken in the "far field" (i.e., at distances of the order of $10 \mathrm{~m}$ or greater away from facility excavations). Measurements in salt within a few meters of mined faces typically yield smaller estimates of the pore pressure. Beauheim et al. (1991) inferred formation pressures from pressure-pulse tests in boreholes drilled from Room C2 in a setting similar to that of Room D. Pressures in three sections within the first few meters below the floor were inferred to be $0.5,3.2$, and $4.1 \mathrm{MPa}$, suggesting that stress relief due to room presence causes a significant reduction in pore pressure. 
The parameter estimates reported here for the small-scale brine inflow experiments yield values for the product of the permeability and the initial pressure. The permeability has been separated only by making the additional assumption that $p_{\infty}=10 \mathrm{MPa}$. This choice may be appropriate for salt undisturbed by excavation effects, but is likely too large for the salt penetrated by the boreholes under consideration, as suggested above. If a better estimate of $p_{\infty}$ is available, the estimates of $k$ and $C$ reported here can be rescaled accordingly. As noted previously, the adjustment is proportional to the change in the assumed initial pressure. If one takes, for example, $p_{\infty}=5 \mathrm{MPa}$, the estimated permeabilities must be multiplied by a factor of 2 , and the estimated capacitances must also be multiplied by 2 .

\subsubsection{Brine Density}

The data reduction reported here assumed a mass density for the brine of $1.2 \times 10^{3} \mathrm{~kg} / \mathrm{m}^{3}$. Recent direct measurements on samples taken from the study holes indicated 1.224 to $1.240 \times 10^{3}$ $\mathrm{kg} / \mathrm{m}^{3}$ (Howarth et al., 1991) Thus, the volume, flux, and permeability estimates are too large by 2 to $3 \%$. Diffusivity is unaffected, but capacitance, calculated from $C=k / \mu c$, is proportionately too large.

\subsubsection{Brine Viscosity}

The viscosity used in the results reported here is $2.1 \times 10^{-3} \mathrm{~Pa} \cdot \mathrm{s}$, based on direct measurements on brines collected in this study. The viscosity measurements were performed with a capillary viscometer, using brines from hole QPB02, over a range of temperatures. The value cited here is for $28^{\circ} \mathrm{C}$. There is, of course, some uncertainty associated with this value. If the viscosity is larger, the inferred permeabilities must be correspondingly larger in order to obtain the same flow rate at the same pressure gradient. The diffusivity is unaffected, but the capacitance must be made larger along with the permeability in order to keep the diffusivity the same. Similarly, if the viscosity is overestimated, the permeability and capacitance are proportionately overestimated. For example, if the viscosity is estimated to be known to within $\pm 0.1 \times 10^{-3} \mathrm{~Pa} \cdot \mathrm{s}$, this introduces a corresponding uncertainty in the reported permeabilities and capacitances of about $5 \%$. 


\subsection{Expected Capacitance for an Elastic Matrix and Fluid}

It was noted in Section 7.1 that the estimates of the capacitance of the salt inferred in this study are quite large in comparison to values expected on the basis of classical models for elastic constituents. Eleven of the fifteen estimates of $C$ shown in Table 6-1 and Figure 7-3 fall in the range $10^{-10}$ to $10^{-8} \mathrm{~Pa}^{-1}$.

Estimates based on the elastic moduli of the salt and brine are of the order of $10^{-11} \mathrm{~Pa}^{-1}$ (Nowak et al., 1988; Beauheim et al., 1991). Parameters that enter into such estimates are recorded here in Table 7-3 for reference. Use of the moduli shown in Table 7-3 and $\phi_{0}=0.01$ in Equation (15) yields $C \sim 0.8 \times 10^{-11} \mathrm{~Pa}^{-1}$. The calculation is rather insensitive to the estimate of the connected porosity, $\phi_{0}$; for example, the assumption of $\phi_{0}=0.001$ and the same moduli results in $C \sim 0.6 \times 10^{-11} \mathrm{~Pa}^{-1}$.

Table 7-3. Elastic Properties of Salt and Brine

\begin{tabular}{|l|c|c|c|}
\hline \multicolumn{1}{|c|}{ Parameter } & Value & Units & Reference \\
\hline Drained Bulk Modulus, $K$ & 20.7 & GPa & Krieg (1984) \\
Poisson Ratio, v & 0.25 & - & Krieg (1984) \\
Solid Bulk Modulus, $K_{s}$ & 23.5 & $\mathrm{GPa}$ & Sumino and \\
& & & Anderson (1984) \\
Fluid Bulk Modulus, $K_{f}$ & 4.0 & $\mathrm{GPa}$ & $*$ \\
Porosity, $\phi_{0}$ & $0.004-0.01$ & - & Stein and Kimball \\
& & & $(1992)$ \\
\hline
\end{tabular}

* determined from acoustic measurements

Beauheim et al. (1991) report a more detailed compilation of ranges of properties by lithology. They compute weighted averages of the storage based on the fractions of the specific lithologies present in a given test interval. The resulting capacitances fall in the range $(0.8-1.3) \times 10^{-11}$ $\mathrm{Pa}^{-1}$, consistent with the calculation based on the values given in Table 7-3.

As noted in Section 7.1, the capacitances inferred in this study should be regarded as "effective" or "apparent" values. These fits yield large estimates of the capacitance that are difficult to reconcile with the classical model of storage due to elastic compressibility of the porous skeleton and the fluid. Recall that the large estimates of capacitance are a consequence of the relatively large 
time scale that characterizes the observed decay of the flux. This long time scale possibly arises from some mechanism(s) other than diffusive relaxation of pore pressure. Deformation of the salt in the neighborhood of excavations is one obvious candidate source for other time scales that may influence the brine flow (McTigue et al., 1989). It is known, for example, that the salt is subject to rate-dependent creep, crack growth, and other processes that may have a profound influence on brine seepage. These processes can lead to time-dependent relaxation of the mean total stress $\sigma$, inelastic dilatation of the salt, large permeability changes, imbibition of air and consequent multiphase flow, etc. Such coupling may introduce time scales longer than the expected diffusive time scale, which may be reflected in the data.

In this context, it is also important to note that the data collected from January 1990 through June 1991, which were not treated in this report but are summarized in Finley et al. (1992), indicate fluxes that either level off or increase slowly for most of the holes. This response is clearly not in accord with the idealized model used in this study to interpret the earlier data. The explanation may lie with the continued evolution of the state of stress and the microstructure of the salt surrounding the excavations. 


\subsection{References}

Bear, J. 1972. Dynamics of Fluids in Porous Media. New York, NY: American Elsevier Publishing Company.

Beauheim, R.L., G.J. Saulnier, Jr., and J.D. Avis. 1991. Interpretation of BrinePermeability Tests of the Salado Formation at the Waste Isolation Pilot Plant Site: First Interim Report. SAND90-0083. Albuquerque, NM: Sandia National Laboratories.

Blackwell, J. H. 1954. "A Transient-Flow Method for Determination of Thermal Constants of Insulating Materials in Bulk," Journal of Applied Physics. Vol. 25, no. 2, 137-144.

Crank, J. 1975. The Mathematics of Diffusion. 2nd ed. Oxford: Clarendon Press,.

Finley, S.J., D.J. Hanson, and R. Parsons. 1992. Small-Scale Brine Inflow ExperimentsData Report Through 6/6/91. SAND91-1956. Albuquerque, NM: Sandia National Laboratories.

Freeze, R. A. 1975. "A Stochastic-Conceptual Analysis of One-Dimensional Groundwater Flow in Non Uniform Homogeneous Media," Water Resources Research. Vol. 11, no. 5, 725741.

Freeze, R.A., and J.A. Cherry. 1979. Groundwater. Englewood Cliffs, NJ: Prentice-Hall.

Gelbard, F. 1992. Exact Analysis of a Two-Dimensional Model for Brine Flow to a Borehole in a Disturbed Rock Zone. SAND92-1303. Albuquerque, NM: Sandia National Laboratories.

Hills, R.G. 1987. ESTIM: A Parameter Estimation Computer Program. SAND87-7063. Albuquerque, NM: Sandia National Laboratories.

Howarth, S.M., E.W. Peterson, P.L. Lagus, K. Lie, S.J. Finley, and E.J. Nowak,. 1991. "Interpretation of In-Situ Pressure and Flow Measurements of the Salado Formation at the Waste Isolation Pilot Plant," Proceedings of the Society of Petroleum Engineers Rocky Mountain Regional Meeting and Low-Permeability Reservoir Symposium, Denver, CO, April 15-17, 1991. SPE-21840. Richardson, TX: Society of Petroleum Engineers, 355-369. 
Jaeger, J.C., 1958. "The Measurement of Thermal Conductivity and Diffusivity with Cylindrical Probes," Transactions, American Geophysical Union. Vol. 39, no. 4, 708-710.

Jaeger, J.C., and M. Clarke. 1942-1943. "A Short Table of $\int_{0}^{\infty} \frac{e^{-x u^{2}}}{J_{0}^{2}(u)+Y_{0}^{2}(u)} \frac{d u}{u}$," Proceedings of the Royal Society of Edinburgh. Vol. A61, pt. III, 229-230.

Krieg, R.D. 1984. Reference Stratigraphy and Rock Properties for the Waste Isolation Pilot Plant (WIPP) Project. SAND83-1908. Albuquerque, NM: Sandia National Laboratories.

McTigue, D.F. 1986. "Thermoelastic Response of Fluid-Saturated Porous Rock," JGR. Journal of Geophysical Research B. Vol. 91, no. 9, 9533-9542.

McTigue, D.F., S.J. Finley, and E.J. Nowak. 1989. "Brine Transport in Polycrystalline Salt: Field Measurements and Model Considerations," EOS Transactions, American Geophysical Union. Vol. 70, no. 43, 1111.

Nowak, E.J., and D.F. McTigue. 1987. Interim Results of Brine Transport Studies in the Waste Isolation Pilot Plant (WIPP). SAND87-0880. Albuquerque, NM: Sandia National Laboratories.

Nowak, E.J., D.F. McTigue, and R. Beraun. 1988. Brine Inflow to WIPP Disposal Rooms: Data, Modeling, and Assessment. SAND88-0112. Albuquerque, NM: Sandia National Laboratories.

Rice, J.R., and M.P. Cleary. 1976. "Some Basic Stress-Diffusion Solutions for Fluid-Saturation Elastic Porous Media with Compressible Constituents," Reviews of Geophysics and Space Physics. Vol. 14, no. 2, 227-241.

Rutherford, B. 1992. Appendix F: "Statistical Analysis of Brine Inflow Data," Small-Scale Brine Inflow Experiments-Data Report Through 6/6/91. S.J. Finley, D.J. Hanson, and R. Parsons. SAND91-1956. Albuquerque, NM; Sandia National Laboratories. F-2 through F-31. 
Stein, C.L., and K.M. Kimball. 1992. Appendix D: "Water Content and Insoluble Residue Analyses of WIPP Samples," Small-Scale Brine Inflow Experiments-Data Report Through 6/6/91. S.J. Finley, D.J. Hanson, and R. Parsons. SAND91-1956. Albuquerque, NM; Sandia National Laboratories. D-1 through D-8.

Sumino, Y., and O.L. Anderson. 1984. "Elastic Constants of Minerals," Handbook of Pr $\mathrm{v}$. Properties of Rocks. Ed. R.S. Carmichael, Boca Raton, FL: CRC Press, Inc. Vol. l $_{\star}$ 138.

Webb, S.W., 1992. Brine Inflow Sensitivity Study for Waste Isolation Filot Plant Boreholes: Results of One-Dimensional Simulations. SAND91-2296. Albuquerque, NM: Sandia National Laboratories. 


\section{DISTRIBUTION}

\section{Federal Agencies}

US Department of Energy (6)

Office of Civilian Radioactive Waste Management

Attn: Deputy Director, RW-2

Associate Director, RW-10/50

Office of Program and

Resources Management

Office of Contract Business

Management

Director, $\mathrm{RW}-22$

Analysis and Verification

Division

Associate Director, RW- 30

office of systems and

Compliance

Associate Director, RW-40

Office of Storage and

Transportation

Director, RW-4/5

Office of Strategic Planning and International Programs

Office of External Relations

Forrestal Building

Washington, DC 20585

US Department of Energy

Albuquerque Operations office

Attn: National Atomic Museum Library

PO Box 5400

Albuquerque, NM 87185-5400

US Department of Energy (4)

WIPP Project Integration office

Attn: W.J. Arthur III

L.W. Gage

P.J. Higgins

D.A. Olona

PO Box 5400

Albuquerque, NM 87115-5400

US Defartment of Energy (2)

WIPP Project Integration Satellite

office

Attn: R. Batra

$R$. Becker

PO Box 3090, Mail Stop 525

Carlsbad, NM 88221-3090

US Department of Energy

Research \& Waste Management Division

Attn: Director

PO BoX E

Oak Ridge, TN 37831
US Department of Energy (3)

WIPP Project Site Office (Carlsbad)

Attn: V. Daub

$\mathrm{J}$. Lippis

J.A. Mewhinney

PO Box 3090

Car1sbad, NM 88221-3090

US Department of Energy

Attn: E. Young

Room E-178

GAO/RCED/GTN

Washington, DC 20545

US Department of Energy

Office of Environmental Restoration and Waste Management

Attn: J. Lytle, EM-30, Trevion II

Washington, DC 20585-0002

US Department of Energy (3)

Office of Environmental Restoration and Waste Management

Attn: M. Frei, EM-34, Trevion II

Washington, DC 20585-0002

US Department of Energy

Office of Environmental Restoration and Waste Management

Attn: S. Schneider, EM-342, Trevion II

Washington, DC 20585-0002

US Department of Energy (2)

Office of Environment, Safety and Health

Attn: C. Borgstrom, EH-25

R. Pelletier, EH-231

Washington, DC 20585

US Department of Energy (2)

Idaho Operations office

Fue 1 Processing and Waste

Management Division

785 DOE Place

Idaho Falls, ID 83402

US Environmental Protection

Agency (2)

Radiation Protection Programs

Attn: M. Oge

ANR -460

Washington, DC 20460 
US Geological Survey (2)

Water Resources Division

Attn: R. Livingston

4501 Indian School NE

Suite 200

Albuquerque, NM 87110

US Nuclear Regulatory Commission

Division of Waste Management

Attn: H. Marson

Mal1 Stop 4-H-3

Washington, DC 20555

\section{Boards}

Defense Nuclear Facilities Safety Board

Attn: D. Winters

625 Indiana Ave. NW, Suite 700

Washington, DC 20004

Nuclear Waste Technical Review

Board (2)

Attn: Chairman

$$
\text { S.J.S. Parry }
$$

1100 Wilson Blvd., Suite 910

Arlington, VA 22209-2297

Advisory Committee on Nuclear

Waste

Nuclear Regulatory Commission

Attn: R. Major

7920 Norfolk Ave.

Bethesda, MD 20814

\section{State Agencies}

Environmental Evaluation Group (3)

Attn: Library

7007 Wyoming NE, Suite F-2

Albuquerque, NM 87109

NM Bureau of Mines and Mineral

Resources

Socorro, NM 87801

NM Energy, Minerals, and Natural

Resources Department

Attn: Library

2040 S. Pacheco

Santa Fe, NM 87505

NM Environment Department (3)

Secretary of the Environment

Attn: J. Espinosa

$1190 \mathrm{st}$. Francis Drive

Santa Fe, NM 87503-0968
NM Environment Department

WIPP Project Site

Attn: P. McCasland

PO Box 3090

Carlsbad, NM 88221

\section{Laboratories/Corporations}

Battelle Pacific Northwest

Laboratories

Attn: R.E. Westerman

MSIN P8-44

Battelle Blvd.

Richland, WA 99352

INTERA Inc.

Attn: J.F. Pickens

6850 Austin Center Blvd.

Suite 300

Austin, TX 78731

INTERA Inc.

Attn: W. Stensrud

PO Box 2123

Carlsbad, NM 88221

IT Corporation

Attn: R.F. Mckinney

Regional office

5301 Central NE

Suite 700

Albuquerque, NM 87108

Los Alamos National Laboratory

Attn: B. Erdal, INC-12

PO Box 1663

Los Alamos, NM 87544

$\mathrm{RE} / \mathrm{SPEC}$, Inc

Attn: W. Coons

4775 Indian School $\mathrm{NE}$

Suite 300

Albuquerque, NM 87110-3927

RE/SPEC, Inc.

Attn: J.L. Ratigan

PO Box 725

Rapid City, SD 57709

Southwest Research Institute (2)

Center for Nuclear Waste Regulatory

Analys is

Attn: P.K. Nair

6220 Culebra Road

San Antonio, TX 78228-0510 
SAIC

Attn: H.R. Pratt

10260 Campus Point Dr.

San Diego, CA 92121

SAIC (2)

Attn: M. Davis

J. To11ison

2109 Air Park Rd. SE

Albuquerque, NM 87106

Tech Reps Inc. (3)

Attn: J. Chapman

C. Crawford

T. Peterson

5000 Marble NE, Suite 222

Albuquerque, NM 87110

TRW Environmental Safety Systems

Attn: L. Wildman

2650 Park Tower Dr., Suite 1300

Vienna, VA 22180-7306

Westinghouse Electric Corporation (5)

Attn: Library

C. Cox

L. Fitch

B.A. Howard

R. Kehrman

PO Box 2078

Carlsbad, NM 88221

Westinghouse-Savannah River

Technology Center (4)

Attn: N. Bibler

J.R. Harbour

M.J. Plodinec

G.G. Wicks

Aiken, SC 29802

\section{National Academy of Sciences, WIPP Panel}

Howard Adler

Oak Ridge Associated Universities

Medical Sciences Division

PO Box 117

Oak Ridge, TN 37831-0117

Ina Alterman

Board on Radioactive Waste

Management, GF456

2101 Constitution Ave.

Washington, DC 20418

Fred $M$. Ernsberger

250 01d Mill Road

Pittsburgh, PA 15238
John D. Bredehoeft

Western Region Hydrologist

Water Resources Division

US Geological Survey (M/S 439)

345 Middlefield Road

Men1o Park, CA 94025

Rodney C. Ewing

Department of Geology

University of New Mexico

Albuquerque, NM 87131

Charles Fairhurst, Chairman

Department of $\mathrm{Civil}$ and Mineral

Engineering

University of Minnesota

500 Pillsbury Dr. SE

Minneapolis, MN 55455-0220

B. John Garrick

PLG Incorporated

4590 MacArthur Blvd., Suite 400

Newport Beach, CA 92660-2027

Leonard F . Konikow

US Geological Survey

431 National Center

Reston, VA 22092

Carl A. Anderson, Director

Board on Radioactive Waste Management

National Research Council

HA 456

2101 Constitution Ave. NW

Washington, DC 20418

Jeremiah 0'Driscoll

Jody Incorporated

505 Valley Hill Drive

Atlanta, GA 30350

Christopher G. Whipple

Clement International

160 Spear St., Suite 1380

San Francisco, CA 94105

Individuals

P. Drez

8816 Cherry Hills Rd. NE

Albuquerque, NM 87111

D.W. Powers

Star Route Box 87

Anthony, TX 79821 


\section{Universities}

R.G. Hills

Department of Mechanical

Engineering

New Mexico State University

Las Cruces, NM 88003

D.F. McTigue (10)

Department of Geological Sciences

AJ - 20

University of Washington

Seattle, WA 98195

University of New Mexico

Geology Department

Attn: Library

Albuquerque, NM 87131

University of Washington

College of Ocean and

Fishery Sciences

Attn: G.R. Heath

583 Henderson Ha11

Seattle, WA 98195

\section{Libraries}

Thomas Brannigan Library

Attn: D. Dresp

$106 \mathrm{~W}$. Hadley St.

Las Cruces, NM 88001

Government Publications Department

Zimmerman Library

University of New Mexico

Albuquerque, NM 87131

New Mexico Junior College

Pannell Library

Attn: R. Hill

Lovington Highway

Hobbs, NM 88240

New Mexico State Library

Attn: N. McCallan

325 Don Gaspar

Santa Fe, NM 87503

New Mexico Tech

Martin Speere Memorial Library

Campus Street

Socorro, NM 87810

WIPP Public Reading Room

Carlsbad Public Library

Attn: Director

$101 \mathrm{~S}$. Halagueno St.

Carlsbad, NM 88220

\section{Foreign Addresses}

Studiecentrum Voor Kernenergie

Centre d'Énergie Nucléaire

Attn: A. Bonne

SCK/CEN Boeretang 200

B-2400 Mol, BELGIUM

Atomic Energy of Canada, Ltd. (3)

Whiteshell Laboratories

Attn: B. Goodwin

M. Stevens

D. Wushke

Pinewa, Manitoba, CANADA ROE 1LO

Francois Chenevier (2)

ANDRA

Route du Panorama Robert Schumann

B.P. 38

92266 Fontenay-aux-Roses, Cedex

FRANCE

Jean-Pierre 01ivier

OECD Nuclear Energy Agency

Division of Radiation Protection and

Waste Management

38, Boulevard Suchet

75016 Paris, FRANCE

Claude Sombret

Centre d'Études Nucléaires de 1a

Vallee Rhone CEN/VALRHO

S.D.H.A. B.P. 171

30205 Bagnols-Sur-Ceze, FRANCE

Gesellschaft fur Reaktorsicherheit

(GRS) (2)

Attn: B. Baltes

W. Muller

Schwertnergasse 1

D-5000 Cologne, GERMANY

Bundesanstalt fur Geowissenschaften und Rohstoffe

Attn: $M$. Langer

Postfach 510153

3000 Hanover 51, GERMANY

Bundesministerium fur Forschung und Technologie

Postfach 200706

5300 Bonn 2, GERMANY

Institut fur Tieflagerung (2)

Attn: K. Kuhn

Theodor-Heuss-Strasse 4

D-3300 Braunschweig, GERMANY 
Physikalisch-Technische Bundesanstalt Attn: P. Brenneke

Postfach 3345

D-3300 Braunschweig, GERMANY

Shingo Tashiro

Japan Atomic Energy Research Inst. Tokai-Mura, Ibaraki-Ken, 319-11

JAPAN

Netherlands Energy Research

Foundation ECN

Attn: L.H. Vons

3 Westerduinweg

PO Box 1

1755 ZG Petten

THE NETHERLANDS

Svensk Kärnbränsleforsorjning $A B$

Attn: F. Karlsson

Project KBS (Kärnbränslesakerhet)

Box 5864

S-102 48 Stockholm

ISWEDEN

Nationale Genossenschaft für die

Lagerung radioaktiver Abfälle (2)

At:n: S. Vomvoris

P. Zuidema

Hardstrasse 73

CH-5430 Wettingen

SWITZERLAND

AEA Technology

Attn: J.H. Rees

D5W/29 Culham Laboratory

Abington, Oxfordshire 0X14 3DB

UNITED KINGDOM

AEA Technology

Attn: W.R. Rodwe11

044/A31 Winfrith Technical Centre

Dorchester, Dorset DT2 8DH

UNITED KINGDOM

AEA Technology

Attn: J.E. Tinson

B4244 Harwe11 Laboratory

Didcot, Oxfordshire OX11 ORA

UNITED KINGDOM

D.R. Knowles

British Nuclear Fuels, plc

Risley, Warrington, Cheshire WA3 6AS

1002607 UNITED KINGDOM

\section{Internal}

1500

1502

1513

4511

6000

6115

6115

6115

6119

6119

6121

6121

6300

6302

6303

6303

6305

6305

6306

6342

6342

6343

6343

6345

6345

6347

6348

6348

6351

6352

6352

7141

7151

$7613-2$

8523-2
D.J. McCloskey

J.C. Cummings

R.D. Skocypec

D.P. Garber

D.L. Hartley

P.B. Davies

J.A. Foesch (10)

Staff (14)

F. Gelbard

Staff (7)

J.R. Tillerson

Staff (7)

D.E. Ellis

L.E. Shephard

S.Y. Pickering

W.D. Weart

S.A. Goldstein

A.R. Lappin

A.L. Stevens

D.R. Anderson

Staff (20)

V. Harper-Slaboszewicz

Staff (2)

R.C. Lincoln

Staff (9)

D.R. Schafer

J.T. Holmes

Staff (4)

R.E. Thompson

S.E. Sharpton

WIPP Central Files (10)

Technical Library (5)

Technical Publications

Document Processing for DOE/OSTI (10)

Central Technical Files

* U.S. GOVERNMENT PRINTING OFFICE 1993-573-110/80268 

